\title{
RF coils for high resolution imaging of the human visual cortex at ultra-high fields
}

Citation for published version (APA):

Sengupta, S. (2018). RF coils for high resolution imaging of the human visual cortex at ultra-high fields. [Doctoral Thesis, Maastricht University]. Ipskamp Printing BV. https://doi.org/10.26481/dis.20181128ss

Document status and date:

Published: 01/01/2018

DOI:

$10.26481 /$ dis.20181128ss

Document Version:

Publisher's PDF, also known as Version of record

\section{Please check the document version of this publication:}

- A submitted manuscript is the version of the article upon submission and before peer-review. There can be important differences between the submitted version and the official published version of record.

People interested in the research are advised to contact the author for the final version of the publication, or visit the DOI to the publisher's website.

- The final author version and the galley proof are versions of the publication after peer review.

- The final published version features the final layout of the paper including the volume, issue and page numbers.

Link to publication

\footnotetext{
General rights rights.

- You may freely distribute the URL identifying the publication in the public portal. please follow below link for the End User Agreement:

www.umlib.nl/taverne-license

Take down policy

If you believe that this document breaches copyright please contact us at:

repository@maastrichtuniversity.nl

providing details and we will investigate your claim.
}

Copyright and moral rights for the publications made accessible in the public portal are retained by the authors and/or other copyright owners and it is a condition of accessing publications that users recognise and abide by the legal requirements associated with these

- Users may download and print one copy of any publication from the public portal for the purpose of private study or research.

- You may not further distribute the material or use it for any profit-making activity or commercial gain

If the publication is distributed under the terms of Article $25 \mathrm{fa}$ of the Dutch Copyright Act, indicated by the "Taverne" license above, 
Dissertation

\section{RF COILS FOR HIGH RESOLUTION IMAGING OF THE HUMAN VISUAL CORTEX AT ULTRA-HIGH FIELDS}

Shubharthi Sengupta

2018 



\section{RF COILS FOR HIGH RESOLUTION IMAGING OF THE HUMAN VISUAL CORTEX AT ULTRA-HIGH FIELDS}

\section{Dissertation}

To obtain the degree of Doctor at Maastricht University, on the authority of the Rector Magnificus, Prof.dr. R.M. Letschert, in accordance with the decision of the Board of Deans, to be defended in public

on Wednesday, November 28, 2018, at 10:45 hours

$$
\text { by }
$$

Shubharthi Sengupta 


\section{Promotor}

Prof.dr. Rainer Goebel

\section{Copromotor}

Dr. Alard Roebroeck

Dr. Gregor Adriany

\section{Assessment Committee}

Prof.dr. Peter de Weerd, Maastricht University (Chair)

Dr. Kâmil Uludağ, Maastricht University

Dr. Federico de Martino, Maastricht University

Prof.dr. Jürgen Hennig, University Medical Center Freiburg

Prof.dr. Klaus Scheffler, Max Planck Institute for Biological

Cybernetics

(C) Shubharthi Sengupta, Maastricht 2018.

All rights reserved. No part of this publication may be reproduced, stored in a retrieval system or transmitted in any form or by any means, electronic, mechanical, photocopying, recording or otherwise, without prior written permission of the author.

Cover Bogna Zajdel, 2018

Production Ipskamp Printing

ISBN 978-94-028-1278-7 
To my parents

তালগাছ এক পায়ে দাঁড়িয়ে, সব গাছ ছাড়িয়ে

উঁকি মারে আকাশে।

মনে সাধ, কালো মেঘ ফুঁড়ে যায়, একেবারে উড়ে যায়;

কোথা পাবে পাখা সে?

তাই তো গে ঠিক তার মাথাতে, গোল গোল পাতাতে

ইচ্ছাটি মেলে তার,

মনে মনে ভাবে, বুঝি ডানা এই, উড়ে যেতে মানা নেই

বাসাখানি ফেলে তার। 



\section{Contents}

1 Introduction 1

1.1 A Brief History of NMR . . . . . . . . . . . . . 3

1.2 Principles of Magnetic Resonance Imaging . . . . . . . . 5

1.3 The MRI system $\ldots \ldots \ldots \ldots \ldots$. . . . . . . . . . . . 9

1.4 The Radiofrequency Coil . . . . . . . . . . . . . . . . . . . 12

1.5 Multi-transmit coils for high field MRI . . . . . . . . . . . 18

1.6 Phased arrays for MRI . . . . . . . . . . . . . . . . . 21

1.7 Electromagnetic Field Simulations . . . . . . . . . . . 23

1.8 RF Safety and SAR $\ldots \ldots \ldots \ldots$. . . . . . . . . . . . 24

1.9 Objective and overview of this thesis . . . . . . . . 26

2 A specialized multi-transmit head coil for high resolution

2.1 Introduction . . . . . . . . . . . . . . . . . . . . . . . .

2.2 Methods . . . . . . . . . . . . . . . . . 35

2.3 Results . . . . . . . . . . . . . . . . . . 46

2.4 Discussion $\ldots \ldots \ldots \ldots \ldots$

3 High resolution anatomical and quantitative MRI of the entire human occipital lobe ex vivo at 9.4T 65

3.1 Introduction $\ldots \ldots \ldots \ldots \ldots$. . . . . . . . 69

3.2 Methods . . . . . . . . . . . . . . . . . . 71 
3.3 Results . . . . . . . . . . . . . . . . . . . . . . . . . 79

3.4 Discussion $\ldots \ldots \ldots \ldots \ldots$. . . . . . . . . . . . 88

4 High resolution MRI neuroanatomy in intact human brains post mortem with a specialized 9.4T RF-coil 93

4.1 Introduction $\ldots \ldots \ldots \ldots \ldots \ldots$

4.2 Methods . . . . . . . . . . . . . . . . . . . 997

4.3 Results . . . . . . . . . . . . . . . . . . . . . . 103

4.4 Discussion . . . . . . . . . . . . . . . . . . . . . . . . . 113

5 A modular, planar RF coil for high resolution, post-mortem imaging at 9.4T 119

5.1 Introduction . . . . . . . . . . . . . . . . . . 121

5.2 Methods . . . . . . . . . . . . . . . . 122

5.3 Results . . . . . . . . . . . . . . . . . . . . . . . . . 129

5.4 Discussion . . . . . . . . . . . . . . . 132

6 Summary and Discussion 135

6.1 Summary . . . . . . . . . . . . . . . . . . 137

6.2 Discussion . . . . . . . . . . . . . . . . . . . 140

\begin{tabular}{lll}
\hline 7 & Valorisation & 149
\end{tabular}

8 Acknowledgments 155

9 Curriculum Vitae 159

$\begin{array}{ll}10 \text { Publications } & 161\end{array}$

\begin{tabular}{ll}
\hline Bibliography & 167
\end{tabular} 


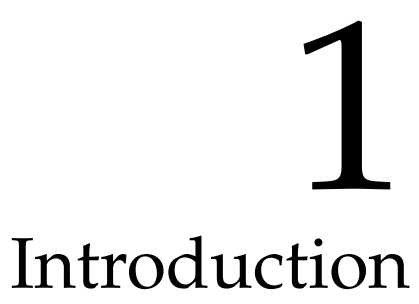

Contents

1.1 A Brief History of NMR $\ldots \ldots \ldots \ldots$

1.2 Principles of Magnetic Resonance Imaging . . . . 5

1.3 The MRI system . . . . . . . . . . . . . . . . . . . . 9 9

$1.4 \quad$ The Radiofrequency Coil . . . . . . . . . . . . . . 12

1.5 Multi-transmit coils for high field MRI . . . . . . 18

1.6 Phased arrays for MRI . . . . . . . . . . . . . 21

1.7 Electromagnetic Field Simulations . . . . . . . . . 23

1.8 RF Safety and SAR . . . . . . . . . . . . . . . 24

1.9 Objective and overview of this thesis . . . . . . 26 


\subsection{A Brief History of NMR}

Magnetic resonance imaging (MRI) has become an indispensable tool when it comes to non-invasive imaging of the human body, for both medical and research purposes. The principle of nuclear magnetic resonance (NMR) forms the basis of every MRI experiment, and was first described by Isidor Isaac Rabi [1]. A few years later both Felix Bloch [2] and Edward Purcell [3] observed and documented the phenomenon of Nuclear Magnetic Resonance (NMR) using condensed matter, simultaneously and independent of each other. Their efforts led to them being awarded the Nobel Prize in Physics in 1952.

Applications of NMR for medical purposes were showcased by Damadian in 1971 by studying the differences in relaxation times $\left(T_{1}\right.$ and $\left.T_{2}\right)$ between healthy and cancerous tissue [4]. In 1973, Lauterbur utilised the principles of NMR to develop a new imaging technique he coined "Zeugmatography" (Zeugmo - to join), referring to the joining together of a weak gradient magnetic field with the stronger main magnetic field allowing the spatial localisation of two test tubes of water [5]. In 2003, Lauterbur [6] and Mansfield [7] - another pioneering figure in the field - were awarded the Nobel Prize in Physics. Magnetic resonance imaging (MRI) has since established itself as a standard in clinical imaging. The development of functional imaging has now made MRI an indispensable tool in physiology and psychology, while allowing researchers to not only study the structure, but the functioning of the human brain.

Magnetic field strengths have also come a long way since the days of Bloch, when he demonstrated "nuclear induction" using a $0.18 \mathrm{~T}$ static magnetic field. The first clinical MR systems installed were no stronger than 0.5T [8]. Over the course of the next 2 decades, $1 \mathrm{~T}$ and $1.5 \mathrm{~T}$ systems remained the workhorses in the clinical domain. At around the same time, the development of 3T systems allowed for higher Signal-to-Noise ratio (SNR) since SNR scales almost linearly with field strength. This development would lead to reduced scan times and 
higher spatial resolutions. Since then, magnetic fields strengths have only increased, with 7T becoming the new imaging standard across MR research areas at several locations worldwide [9, 10, 11]. Presently, a handful of sites have magnets functioning at 9.4T and above, with the sole purpose of increased SNR and reduced scanning times [12]. However, imaging at these high fields is not without its disadvantages. In addition to $B_{0}$ inhomogeneity [13, 14], strong transmit $B_{1}$ inhomogeneities remain at $7 \mathrm{~T}$ and above, as the proton resonant wavelength is shorter than the dimensions of the human body areas being imaged, making it more difficult to produce a uniform RF field over the sample volume [13, 15]. That notwithstanding, several recent advancements in software and hardware methods have helped researchers mitigate the issue of field inhomogeneities and tissue heating at 7T, such that it is well on its way to becoming the new clinical standard for MRI [16]. 


\subsection{Principles of Magnetic Resonance Imaging}

Atoms are the basic unit of matter. Atoms in turn, consist of electrons and nuclei. One of the fundamental properties of most nuclei is spin. Spin is a form of angular momentum, not produced by a rotation or movement, but is an intrinsic property of the nucleus itself. The spin angular momentum J of atomic nuclei is quantized, and takes values of the form:

$$
\vec{J}=\hbar \vec{I}=\hbar \sqrt{I(I+1)}
$$

where $\vec{I}$ is the spin momentum (vector) and $I$ is the quantum number (scalar). The spin angular momentum $\vec{J}$ has a magnetic dipole moment $\vec{\mu}$ :

$$
\vec{\mu}=\gamma \vec{J}=\gamma \hbar \vec{I}
$$

where $\gamma$ is the gyromagnetic ratio. The gyromagnetic ratio can have a positive or a negative value, depending on the nuclear isotope. In the absence of a magnetic field, the orientation of $\vec{\mu}$ is random and the orientation distribution of a collection of spins is isotropic

The value of the gyromagnetic ratio $\gamma$ is different for every element in the periodic table, and nuclei with a large value of $\gamma$ are most easily detected in NMR experiments. Also, almost every element has an isotope with non-zero nuclear spin. The ${ }^{1} H$ nucleus is the most frequently used isotope in NMR imaging and spectroscopy, due to its large $\gamma$ and easy availability in nature.

The angular momentum is a vector quantity $\vec{J}=\left(J_{x}, J_{y}, J_{z}\right)$. The $\mathrm{z}$ 
component of $\mathrm{J}$, in a magnetic field $\vec{H}=\left(0,0, H_{z}\right)$, will be quantized:

$$
J_{z}=m_{I} \frac{h}{2 \pi}
$$

where $m_{I}=-\mathrm{I},-\mathrm{I}+1 . ., 0 \ldots, \mathrm{I}-1, \mathrm{I}, \mathrm{I}$ is the total spin number and $h$ is Planck's constant, where

$$
\hbar=\frac{h}{2 \pi}
$$

When placed in a static external magnetic field $\vec{H}=\left(0,0, H_{z}\right)$, nuclei with nuclear spin $I$ can adopt one of the spin states $(1 / 2$ or $-1 / 2$ for $\mathrm{I}=1 / 2$ and 1,0 or -1 for $\mathrm{I}=1$ ) corresponding to possible orientations of the magnetic moment $\mu_{I}$ (parallel or anti-parallel to $H_{0}$, in the case of $\mathrm{I}=1 / 2$ ). For $\mathrm{I}=1 / 2$, two spin energy levels exist. They are referred to spin-up and spin-down states, or $\alpha$ and $\beta$ states respectively.

The energy difference between the 2 states is:

$$
E=-\frac{h \gamma \mu_{0} \mu_{r} H_{0}}{2 \pi}
$$

where $\mu_{0}$ and $\mu_{r}$ are the intrinsic and relative permeability. The equation can thus be rearranged into:

$$
E=-\hbar \gamma B_{0}
$$

where $B_{0}$ is the magnetic flux density.

The nuclei can undergo transition between the upper and lower en- 
ergy levels, by absorbing or emitting a photon. Particles in the lower energy states absorb a photon and migrate to the next energy level. The energy of this absorbed photon must be equal to the difference of the two energy states. The energy of a photon is $E_{p}=h v$, where $v$ is the frequency of the photon. Hence,

$$
E_{p}=E \Rightarrow h v_{0}=-\hbar \gamma B_{0}
$$

Taking the second part of the equation and solving it, we get:

$$
2 \pi v_{0}=\omega_{0}=-\gamma B_{0}
$$

where $\omega_{0}$ is referred to as the resonant frequency or the Larmor frequency. The negative sign indicates that the magnetic energy is lowest if the magnetic moment is parallel to the $B_{0}$ field. Apart from the externally applied magnetic field, the spins experience the very small field fluctuations of the surrounding nuclear spins, which influences the orientation of the spin.

Since the environment has a finite temperature, it is slightly more probable that the nuclear spin is driven towards an orientation with low magnetic energy than towards an orientation with high magnetic energy. When samples are placed in a magnetic field, over time, a few more nuclei fall in the $\alpha$ state. This excess of spins in the $\alpha$ over the $\beta$ states accounts for the entire net magnetization that is used in an NMR experiment. The difference in the number of spins in the two states, as given by a Boltzmann distribution, is:

$$
\Delta N=\frac{N \hbar \gamma B_{0}}{2 \kappa T_{s}}
$$


where $N$ represents the number of protons, $T_{s}$ is the temperature of the sample in Kelvin and $\kappa$ stands for the Boltzmann's constant. Given that the magnetic moment of each proton is $\frac{\gamma \hbar}{2}$, the net magnetisation available for MR signal generation can be written as:

$$
M_{0}=\frac{N \hbar^{2} \gamma^{2} B_{0}}{4 \kappa T_{s}}
$$

This equation posits that the net magnetisation available for MR signal generation is therefore directly proportional to the strength of the main magnetic field $B_{0}$, resulting in the availability of more SNR at higher field strengths. 


\subsection{The MRI system}

Fig 1.1 shows a block diagram of a typical MRI scanner system, with details of the individual units listed below.
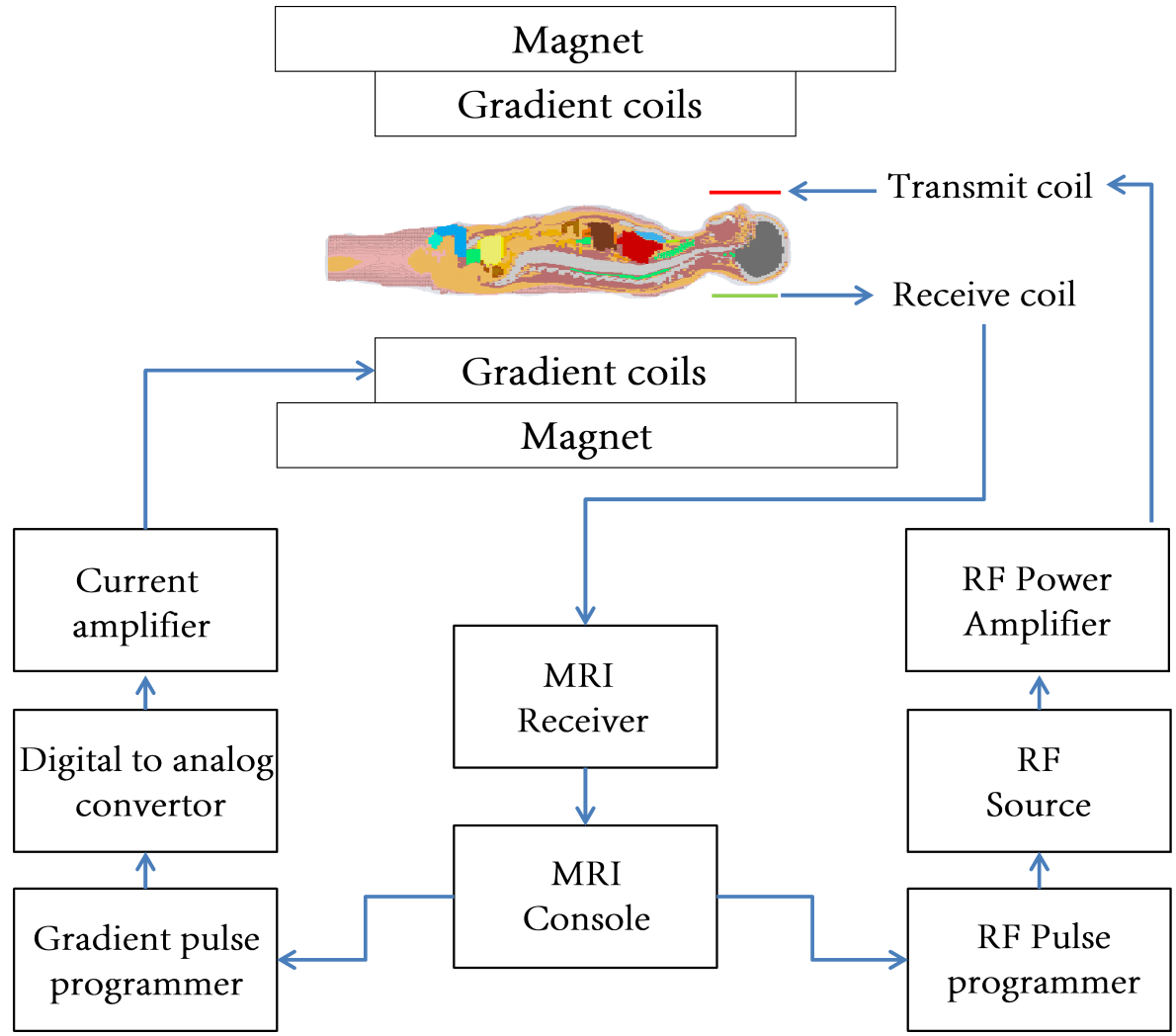

Figure 1.1: Block diagram of an MR imaging system

\section{The Magnet}

The development of wide-bore, superconducting magnets has been crucial towards MR imaging of human subjects at high field strengths. 
The coil windings are constructed using superconducting wire - generally an alloy of niobium-titanium (NbTi) or niobium-tin $\left(\mathrm{Nb}_{3} \mathrm{Sn}\right)$ - as they are capable of conducting much larger electrical currents, resulting in higher magnetic field strengths. The magnet generates the static magnetic field $B_{0}$ which aligns the magnetic moments of the nuclei of the sample, producing a net magnetization. For high resolution MR imaging, it is imperative that the $B_{0}$ field is as homogeneous as possible across the imaging FoV. In this homogeneous $B_{0}$ field, the magnetic moments of the nuclei precess with approximately similar phases and Larmor frequencies, allowing their MR signals to accumulate.

\section{Gradient Coils}

Gradient coils are particularly important for MR imaging as they spatially encode the MR signals by causing a variation in Larmor frequency as a function of their position [6, 7]. The gradient coils generate controlled magnetic field gradients across all 3 axes $(x, y$ and $z)$ and the application of all 3 gradients allows the MR signals from each imaged voxel to have its own unique combination of frequency and phase. Gradient coils can be classified into 3 types, namely: the Frequency Encode Gradient (FEG), the Phase Encode Gradient (PEG) and the Slice Selective Gradient (SSG), and are driven using a pulse programmer, a Digital-to-Analog converter (DAC) and a current amplifier. Gradient coils can typically produce gradients from 20 to 100 $\mathrm{mT} / \mathrm{m}$.

\section{RF Transmitter and Receiver}

The RF transmitter generates a $B_{1}$ magnetic field, orthogonal to $B_{0}$, that flips the net magnetization $M_{0}$ away from the static field $B_{0}$ and towards the transverse plane by an angle $\alpha$, determined by the strength 
and duration $\tau$ of the applied $B_{1}$ field. The RF transmit system supplies precision-controlled pulses of varying phase and amplitudes onto one or more near-field RF antennas, commonly referred to as RF coils. The RF transmitter signals must be amplified (to $\mathrm{kW}$ levels) in order to sufficiently excite the sample under investigation. The amplification is achieved through the use of RF power amplifiers (RFPA). RFPAs consist of RF driver stages, amplifiers and directional couplers which help in monitoring the output power levels. Typical RFPAs in use can provide up to several $\mathrm{kW}$ of power.

Once the RF excitation pulse has played out, the nuclei start releasing the absorbed energy. A receive $(\mathrm{Rx})$ coil placed in close proximity to the sample picks up this energy and produces an electrical signal that represents the emitted MR signal. It is then fed into Low Noise Amplifiers (LNAs) that form the first amplification stage of the signals, and are therefore often referred to as pre-amplifiers. Depending upon the size and volume of the sample being imaged, one or more receive coils may be used for MR signal acquisition. Utilising an array of phased receive coils is advantageous as array coils produce superior SNR and can also help in shortening acquisition times through the use of parallel acquisition techniques [17, 18, 19]. Depending upon the type of sample being imaged, a variety of coils - namely loops, dipoles or microstrips - can be used for signal detection [20, 21, 22, 23].

Within the context of receive coils, it would be important to recall the principle of reciprocity [24, 25]. The sensitivity at any point in space, within a coil's FoV during signal reception, is directly proportional to the $B_{1}$ field at that point generated by an unit current passing through the same coil. Therefore, according to the principle of reciprocity, in order to maximise the signal induced across the coil terminals, the $B_{1}$ field incident on the coil should also be maximised. This shows that coils should be designed such that they conform to the geometry and contours of the sample under investigation, as the $B_{1}$ field for any point within the coil's FoV decreases with increasing distance away from the coil surface. 


\subsection{The Radiofrequency Coil}

The RF coil acts as the primary interface between the subject/sample being imaged and the magnet. As the MR signal being emitted by the sample is weak, sufficient care must be taken when designing a receive coil. Fundamentally, the RF coil is an inductor which is sensitive to a magnetic field. Using Biot-Savart's Law, the magnetic field of an RF coil can be calculated as:

$$
\vec{B}(\vec{r})=\frac{\mu_{0} I}{4 \pi} \int \frac{d \vec{l} \times \vec{r}}{|r|^{3}}
$$

where $\mu_{0}$ is the permeability constant in vacuum and $I$ the current in the differential element $d \vec{l}$.

The net magnetisation of the sample, as discussed in the preceding sections, must be tipped away from its state of equilibrium and into the transverse or $x y$ plane, in order to generate the FID (Free Induction Decay) signal. This is achieved by the application of an RF, or $B_{1}$ field oscillating at the relevant Larmor frequency, perpendicular to the main magnetic field. This $B_{1}$ field can be divided into two counterrotating components, namely $B_{1}^{+}$and $B_{1}^{-}$, also known as the transmit and receive fields respectively [25]. While $B_{1}^{+}$is the field component that rotates with the precession of the magnetic moment, the $B_{1}^{-}$component rotates in the opposite direction, both at the resonant Larmor frequency $\omega_{0}$. It can be written as:

$$
\vec{B}_{1}^{+}=\frac{\vec{B}_{1, x}+i \vec{B}_{1, y}}{2}
$$

and

$$
\vec{B}_{1}^{-}=\frac{\left(\vec{B}_{1, x}-i \vec{B}_{1, y}\right)^{*}}{2}
$$


where $\vec{B}_{1}^{+}, \vec{B}_{1}^{-}, \vec{B}_{1, x}$ and $\vec{B}_{1, y}$ are all complex vector quantities while * indicates the complex conjugate.

The magnetic field created by an unit current passing through a conductor is defined by Ampere's Law and can be written as:

$$
\oint B \cdot d l=\mu_{0} \int J \cdot d s
$$

where $B$ is the magnetic field and $J$ is the net current density. The power incident from the RFPAs are channelled into the transmit coils, which then conduct the currents required in producing the homogeneous magnetic field $\left(B_{1}^{+}\right)$across the sample under investigation. An RF pulse is applied to tip the net sample magnetisation into the transverse plane by an angle $\alpha$, also known as the flip angle. This can be written as:

$$
\alpha=\int_{0}^{\tau} \gamma B_{1} d t
$$

where $\tau$ is the length of the RF pulse duration.

Once the RF transmit pulse is switched off, the precessing magnetisation in the transverse plane begins relaxing towards its state of equilibrium, i.e., aligned with the main magnetic field $B_{0}$. If another RF coil were to be placed in close proximity to the sample, the relaxing, precessing magnetisation would induce an oscillating voltage signal across the coil terminals. This signal, exponentially decaying and unaffected by any gradient pulses, is know as the FID or Free Induction Decay.

For the aforementioned receive RF coil, we can apply Faraday's Law of Induction, which states that a time-varying magnetic field will induce 
a voltage across the terminals of a closed loop, or in this case, across the terminals for a receive coil. This can be written as:

$$
\xi=\oint E \cdot d l \Rightarrow-\frac{\mathrm{d} \Phi_{s}}{\mathrm{~d} t}=-\frac{\mathrm{d}}{\mathrm{d} t} \int B \cdot d s
$$

where $\xi$ is the induced voltage or EMF, and is proportional to the rate of change of magnetic flux $\Phi_{s}$ through the closed surface.

The energy stored in an inductor $L$ due to current $I$ flowing through it is written as:

$$
U=\frac{I^{2} L}{2}
$$

where $U$ is the energy available to the probe from the decaying MR signal. As mentioned above, the coil isn't an ideal inductor, but also possesses an intrinsic resistance $R$. This generates losses in the form of Ohmic heating, which can be formulated as:

$$
W=\frac{I^{2} R}{2}
$$

The resulting efficiency of the coil can be measured as the ratio of stored to dissipated power, and can be written as

$$
Q=2 \pi \frac{U}{W}=\omega \frac{L}{R}
$$

where $\mathrm{Q}$ is the coil quality factor ( $\mathrm{Q}$-factor) and $\mathrm{R}$ represents the total loss factor. We can thus conclude that reducing the total loss factor $\mathrm{R}$ 
would result in a higher coil Q-factor. When a coil is loaded with a sample, the coil's resonance is dampened and its impedance changes slightly. An important metric to quantify a loaded coil's performance is the Q-ratio, which is the ratio of the unloaded coil Q-factor $\left(Q_{U L}\right)$ vs the loaded coil Q-factor $\left(Q_{L}\right)$. Therefore maximising the $Q_{U L}$ is imperative to coil design. The loss mechanisms, representative of the total loss factor R, can be further enumerated as below:

- Resistive or Ohmic losses : Losses in the coil conductor and/or the components used in the coil assembly are termed as resistive losses $\left(R_{\Omega}\right)$. Skin effect plays a major role here. The skin effect is the tendency of an alternating current to distribute itself over the conductor such that the current density near the surface of the conductor is more than that at the core of the conductor. The skin effect increases the effective resistance of the conductor with increasing frequency and is proportional to $\sqrt{\omega}$ where $\omega$ is the operating frequency. These losses are more pronounced for microcoils as the diameter of wire used for coil construction is very small, leading to much higher skin effect losses [26].

- Radiation losses: Radiation losses can be defined as the RF energy lost to the radiated far field of the coil and can be termed as $R_{r}$. Radiation losses increase proportionally with frequency $\left(\propto \omega^{4}\right)$ and with the surface area $\left(A^{2}\right)$ of the coil in question [27]. This results in decreased transmit efficiency, decreased SNR and increase in power absorbed by the sample. An effective method of reducing radiation losses in loop coils is to add an RF shield at an optimal distance away from the coil, resulting in increased Q-ratio. The Q-factor of an unloaded coil can then be written as:

$$
Q_{U L}=\frac{\omega L}{R_{u}}
$$

where $R_{u}$ is the combined Ohmic resistance $R_{\Omega}$ and radiation 
resistance $R_{r}$ of the coil.

- Tissue and Sample losses: When a coil is loaded with a dielectric sample, additional losses arise from induced eddy currents and displacement currents in the sample volume. While displacement currents can be mitigated by using distributed capacitors on the coil loops, losses generated due to eddy currents induced in the sample is not quite unavoidable. Distributing capacitors of equal value along the loop is a tested method of reducing displacement currents and the electric field buildup around a capacitor, with the capacitors spaced apart by $\lambda / 20$ to $\lambda / 10$, where $\lambda$ is the operating wavelength [28]. If the capacitors are not distributed in such fashion, it may result in reduced unloaded coil quality factor $\left(Q_{U L}\right)$ while also rendering the loop sensitive to changes in the sample or load.
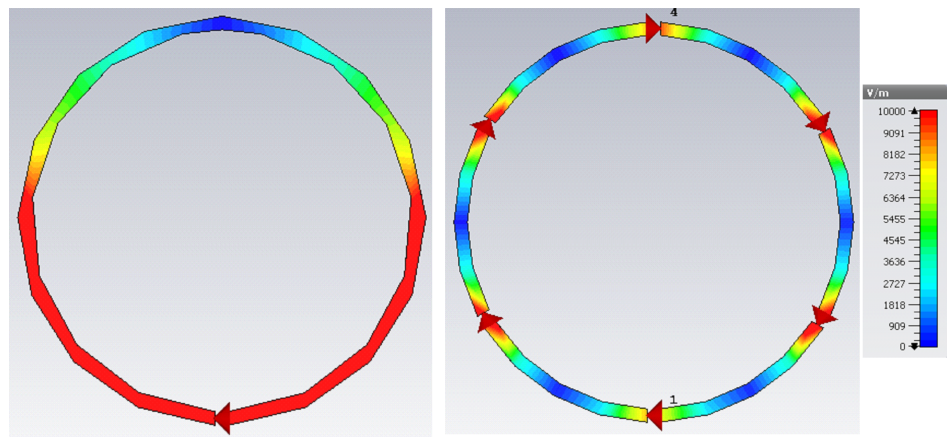

Figure 1.2: E-field distribution comparison simulation at $400 \mathrm{MHz}$ for a $6 \mathrm{~cm}$ diameter loop (L) Loop coil with a single capacitor showing large Efield build-up around capacitor (R) Distributing capacitance equally across the loop results in lower E-fields around capacitors

The corresponding quality factor of a loaded coil with sample resistance $R_{s}$ can be written as

$$
Q_{L}=\frac{\omega L}{R_{u}+R_{s}}
$$


and when the Q-ratio $\left(Q_{U L} / Q_{L}\right)$ is maximum, the coil is dominated by sample noise.

While the inductor/coil isn't self resonant, it is the addition of capacitance that makes the circuit resonant. When the capacitive reactance $\left(X_{C}=-1 / \omega_{0} C\right)$ and the inductive reactance $\left(X_{C}=\omega_{0} L\right)$ are equal to each other, the coil is resonant at frequency $\omega_{0}$. The resonant frequency of the resulting circuit can be written as

$$
\omega_{0}=\frac{1}{\sqrt{L C}}
$$
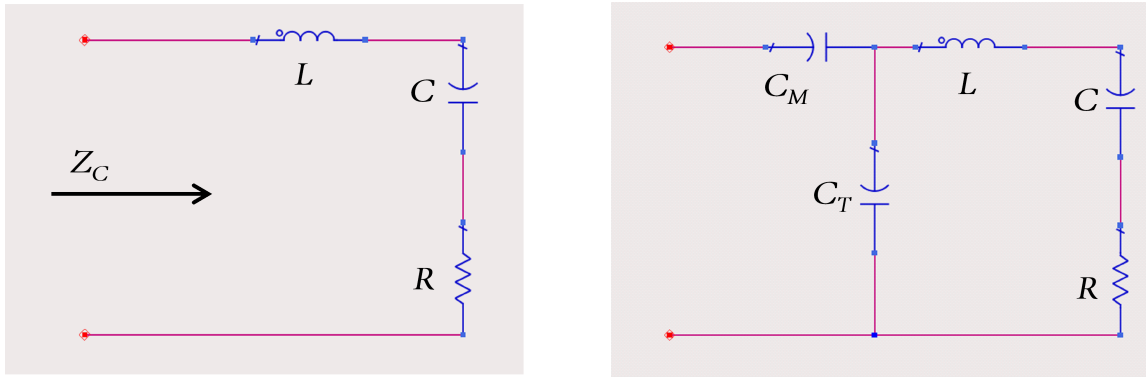

Figure 1.3: (L) RLC resonant circuit with the real and imaginary parts of the impedance $Z_{c}(\mathrm{R})$ The same circuit with added tuning and matching capactiors $C_{T}$ and $C_{M}$ respectively

However, it must be noted, that the RF coil is far from being an ideal inductor. The RF coil acts as an inductor whose impedance can be formulated as:

$$
Z_{c}=R_{c}+j X_{c}
$$

Where $R_{c}$ is the coil resistance (usually in the order of a few Ohms). In order to maximise the power transfer from the coil onto the preamplifers, the coils' output impedance must match the input impedance 
of the preamplifier, i.e the conjugate of the amplifier input impedance $Z_{c}^{*}$ must be equal to the transformed impedance of the coil, which is usually $50 \Omega$ [29].

In order to match a coil's output impedance to $50 \Omega$, a tuning-matching network consisting of a tuning capacitor $\left(C_{T}\right)$ in parallel with the coil and a matching capacitor $\left(C_{M}\right)$ in series with the coil, is implemented.

\subsection{Multi-transmit coils for high field MRI}

With increasing $B_{0}$ field strengths and corresponding Larmor frequencies, the RF wavelength inside tissue approaches the dimensions of the sample being investigated, resulting in transmit artifacts as RF penetration and interference patterns become dominant [9, 30, 31, 32]. This leads to non uniform $B_{1}$ field patterns and a significant difference between transmit and receive $B_{1}$ field profiles [25, 33, 34]. In order to achieve transmit homogeneity in the region of interest, multi-transmit coils capable of localised RF shimming are implemented.

For a volume transmit coil with $n$ current-carrying elements, the RF field produced can be written as

$$
\left|\sum_{i=1}^{n} B_{1 i}^{+}(\vec{r})\right|
$$

where $B_{1}^{+}$denotes the transmit field component, $i$ denotes the individual coil element and $\vec{r}$ denotes the location within the transmit coil's field of view (FoV). The $B_{1}^{+}$fields add constructively, leading to a higher flip angle at the center of the coil, with transmit efficiency decreasing away from the coil center as the $B_{1}^{+}$fields now interact in 
a partially destructive manner. This combination of constructive and destructive interferences in the RF field lead to $B_{1}^{+}$inhomogeneities at high fields.

\section{RF shimming}

If the volume coil mentioned above were to be divided into separate individual current-carrying elements, it would result in a transmit array whereby each element can be driven independently [35, 36, 37]. The net $B_{1}$ field produced by an array of $n$ transmit elements is the complex sum of the fields generated by the $n$ elements, weighted by a complex amplitude $a$, and can be written as:

$\left|\sum_{i=1}^{n} B_{1 i}^{+}(\vec{r})\right|=a_{1} \cdot B_{11}^{+}(\vec{r}) e^{-j \omega t_{1}}+a_{2} \cdot B_{12}^{+}(\vec{r}) e^{-j \omega t_{2}}+\ldots . a_{n} \cdot B_{1 n}^{+}(\vec{r}) e^{-j \omega t_{n}}$

This enables better control over the phase and magnitude of the currents delivered to the transmit coil, and therefore, the subsequent $B_{1}^{+}$ fields generated by each element. The method of controlling the phase and amplitude of the currents supplied to each transmit coil is referred to as RF shimming [38, 39]. RF shimming can be implemented through static and dynamic methods. When static shimming, the transmit elements are phased apart from each other using varying, pre-defined lengths of coaxial cables connected to the feed ports as shown in Fig 1.4, by using power splitters such as a Wilkinson power divider [40] and also through static phase and gain control. In dynamic shimming, each transmit element is connected to its own dedicated RF amplifier, allowing for much finer control over the phase and amplitude of the currents being fed to the coils, as seen in Fig 1.5. This approach is also known as the parallel transmit method (pTx) and when combined 
with optimised gradient waveforms helps achieve better control over $B_{1}^{+}$homogeneity and consequently the distribution of electric fields produced in the tissue under investigation, leading to local SAR reduction [41, 42].

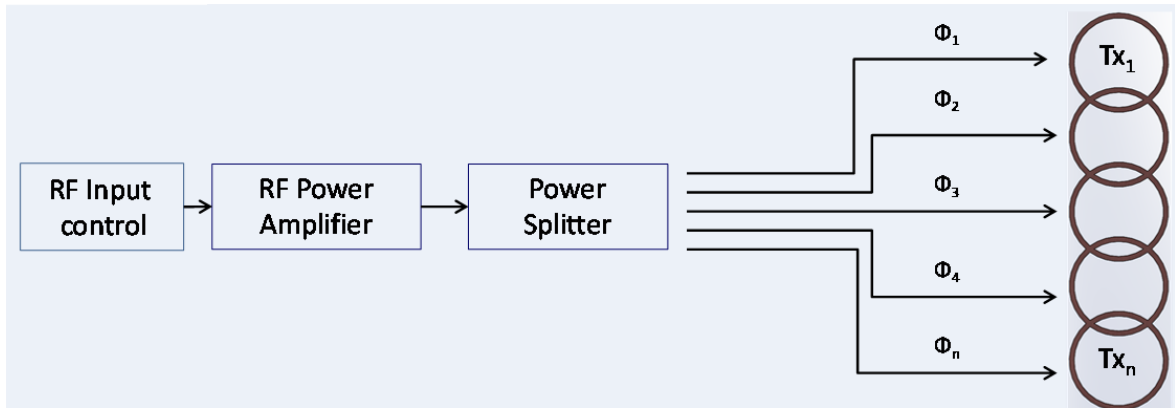

Figure 1.4: Static RF shim Schematic for a static RF shim setup

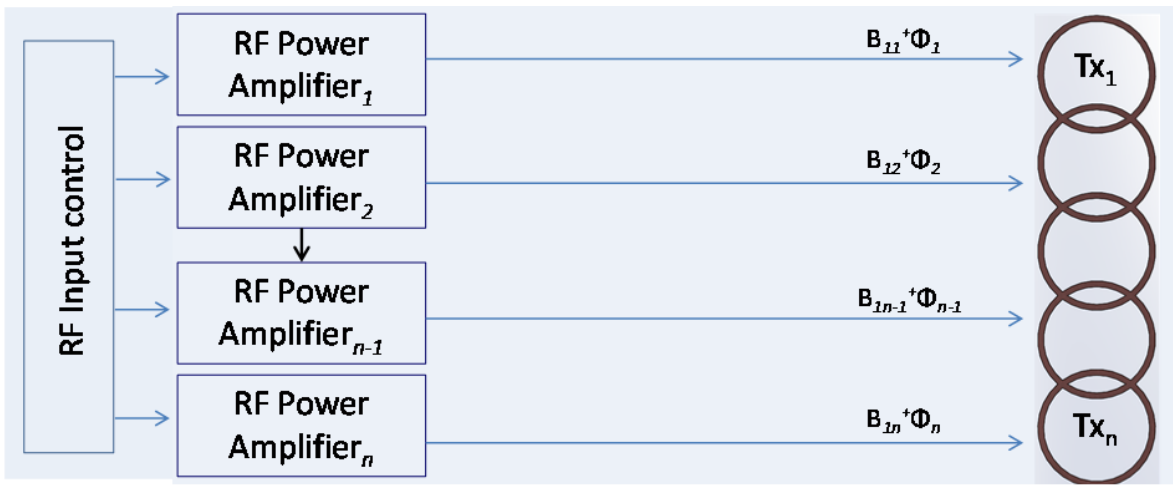

Figure 1.5: Dynamic RF shim Schematic for a dynamic, parallel transmit setup 


\subsection{Phased arrays for MRI}

The introduction of the concept of coil arrays allowed the use of 2 or more localised coils, instead of a single surface coil, for MR signal detection [43]. Provided the two loops are perfectly decoupled wherein the mutual inductances of the two loops are negligible leading to minimal interaction between them - effective scan time could be reduced. The two decoupled loops can be thought of as two independent sources that provide information about a single point/voxel in the coils' combined FoV. Figure 1.6 provides a illustrative example of coil decoupling as a function of the distance between loops.

Roemer reported the first practical use of phased arrays by using overlapped coils ( 0.25 times the coil diameter for circular loops and 0.1 times the width for square loops) to achieve mutual decoupling between neighbouring elements, and employing low input-impedance preamplifiers connected to each loop to further decouple next adjacent phased array elements [21]. The latter method of decoupling is referred to as preamplifier decoupling, a concept that has found use in array coil design and decoupling ever since. Roemer's results showed that the setup could achieve the high SNR associated with small loops across a large FoV when employing a phased array layout, without any penalties to imaging time. Additionally, adjacent loops can be decoupled inductively - whereby 2 inductors (one for each loop) are wound in opposite directions and placed next to each other along their main axis [44, 45]. The physical dimensions of the wound inductors depend on the size and the distance between the loops being decoupled. The use of phased arrays for high resolution imaging has since seen the development of coils for imaging various parts of the body and has gone onto become a standard in commercial MR imaging, leading to the push towards more research into optimising this imaging standard.

In order to harness the localised SNR gain afforded by smaller loops in comparison to larger ones, phased array coils comprising of a variety of channel counts have been developed over time - ranging from a 
16 channel phased array head coil at $1.5 \mathrm{~T}$ by Porter et al. [46] to a 128 channel phased array receive coil by Hardy et al. [47]. Wiggins presented a 32-channel phased array coil for head imaging at 3T, which utilised a soccer-ball geometry of hexagonal and pentagonal elements to provide effective coverage of the head as well as optimal decoupling between coil elements [48]. The 32 channel coil was able to achieve an overall SNR gain of upto 3.5 times that of a commercial head coil, while exhibiting reduced g-factors (or noise amplification factor) for accelerated imaging. This concept was furthered by the 64 channel coil at $3 \mathrm{~T}$ by Keil, where the loops employed were smaller than those of the 32 channel coil [49]. Results showed an increase in SNR by upto 1.3 times that of the previous 32 channel coil, with reduced g-factors. Since then, Wiggins pioneered the dense 96 channel coil array [50], further improving on the available SNR, followed by the 128 channel cardiac coil array by Schmitt [51].

Coil image reconstruction algorithms also play a crucial role in MR imaging as they allow for methods to optimally combine the MR signal data from all the coils in an array in order to generate an image with optimal SNR with reduced scan time. Accelerated imaging reconstruction protocols include SENSE [18] (Sensitivity Encoding for fast MRI), GRAPPA [19] (generalized auto-calibrating partially parallel acquisition), and SMASH [17] (simultaneous acquisition of spatial harmonics), among others.

By increasing the number of individual coils within an MR phased array, we can achieve higher image SNR, reduced scan times via downsampling of k-space and utilising coil sensitivity maps to compensate for said missing samples [18, 52] and finally, by increasing the image FoV while not incurring any time penalties. The main drawbacks related with massively parallel MR arrays are size and cost, and both increase with increasing array sizes, and the added complexity it brings with. 

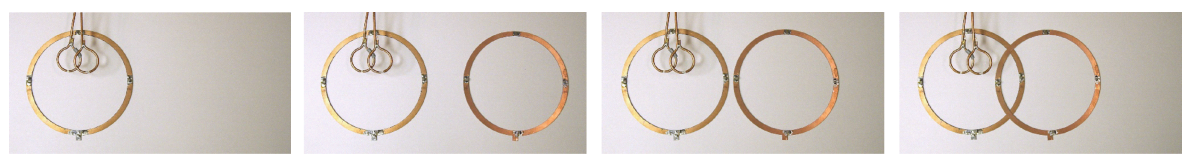

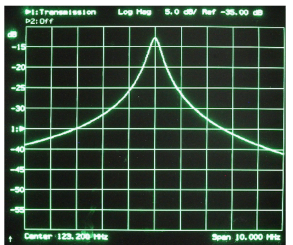

Single coil

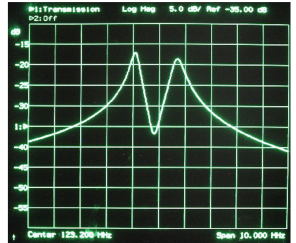

Lightly coupled

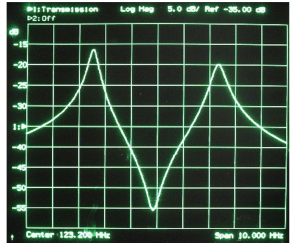

Strongly coupled

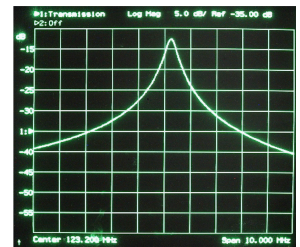

Critical overlap

Figure 1.6: Phased arrays. Illustration of inter-coil coupling with respect to coil separation and overlap [53]

\subsection{Electromagnetic Field Simulations}

In order to determine the interaction between the body being imaged and the RF field generated by the transmit coil, numerical simulations are undertaken. Using complex body voxel models, 3D electromagnetic simulation softwares (CST, HFSS, SEMCAD etc) solve discretized, time dependent Maxwell's equations across the simulation space iteratively while reaching a steady state. While not only aiding in SAR simulations, these softwares also help in optimising specific RF coil designs by generating transmit $B_{1}^{+}$distributions. This is of primary importance at higher fields ( $\geq 7 \mathrm{~T}$ ) as RF wavelengths becomes smaller, with wavelengths in tissue approaching the dimensions of the adult human head at 9.4T. This results in interference effects which leads to transmit inhomogeneities in the FoV. It is therefore crucial to perform transmit field simulations with an emphasis on $B_{1}^{+}$homogeneity, while ensuring a low SAR level in order for RF coil hardware to comply with regulatory guidelines related to energy absoprtion and heating due to exposure to RF fields, in human tissue. As mentioned before, the EM simulations work by solving a discretized model of the RF coil design and tissue under investigation, in either the time domain or the frequency domain. To this effect, the computa- 
tional domain is discretized into smaller, cubical structures called Yee cells, which are used to define the electrical $\left(\bar{E}=E_{x} \bar{x}+E_{y} \bar{y}+E_{z} \bar{z}\right)$ and magnetic $\left(\bar{H}=H_{x} \bar{x}+H_{y} \bar{y}+H_{z} \bar{z}\right)$ field components along the three axes [54]. The propagation of an excitation is then calculated along the 3D grid of the Yee cell using discrete time steps, given certain boundary value conditions.

For solving Maxwell's equations, one can either use the Finite Difference Time Domain (FDTD) method for solving the differential form of the equations, or use the Finite Integration Technique (FIT) method to solve the integral forms of the same. In this work, the simulation software CST (3DS Simulia, Darmstadt, Germany) was used to implement the FDTD method.

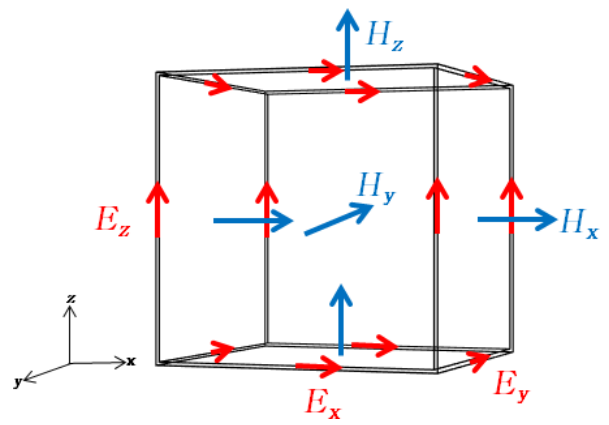

Figure 1.7: The Yee cell. Assignment of the field vector components on an FDTD cell for Yee's algorithm

\subsection{RF Safety and SAR}

During the transmit phase, a portion of the incident power is absorbed by the sample, resulting in heating effects. Care must be taken to restrict the rise in temperature of the subject being imaged. While the exact change in temperature cannot be measured during a scan, an estimation in change in temperature can be approximated by monitoring 
the Specific Absorption Rate (SAR). SAR can be defined as

$$
\mathrm{SAR}=\frac{\text { RF energy dissipated in sample }}{\text { sample weight } \times \text { time }}
$$

The power dissipated, $P$, can be written as

$$
P=\int \frac{\sigma(r)|E(r)|^{2}}{2} d r
$$

where $\sigma$ is the sample conductivity in the electric field $\mathbf{E}$. The resulting SAR can be calculated as

$$
S A R \propto \int \frac{\sigma(r)|E(r)|^{2}}{\rho(r)} d r
$$

and can be simplified to

$$
S A R=\frac{\sigma E^{2}}{\rho}
$$

Standards for SAR limits for MR imaging are set by the International Electrotechnical Commission (IEC) which provide regular and updated guidelines for SAR safety and monitoring. The current IEC normal operating mode limits (according to IEC standard 60601 -2-33) [55] are $4 \mathrm{~W} / \mathrm{kg}$ over the whole body, $3.2 \mathrm{~W} / \mathrm{kg}$ over the head and $10 \mathrm{~W} / \mathrm{kg}$ over any $10 \mathrm{~g}$ sample of tissue, over a duration of 6 minutes and averaged over $10 \mathrm{~g}$ of tissue under investigation. Peak SAR for short durations of 10 seconds are limited to 2 times the stated values. 


\subsection{Objective and overview of this thesis}

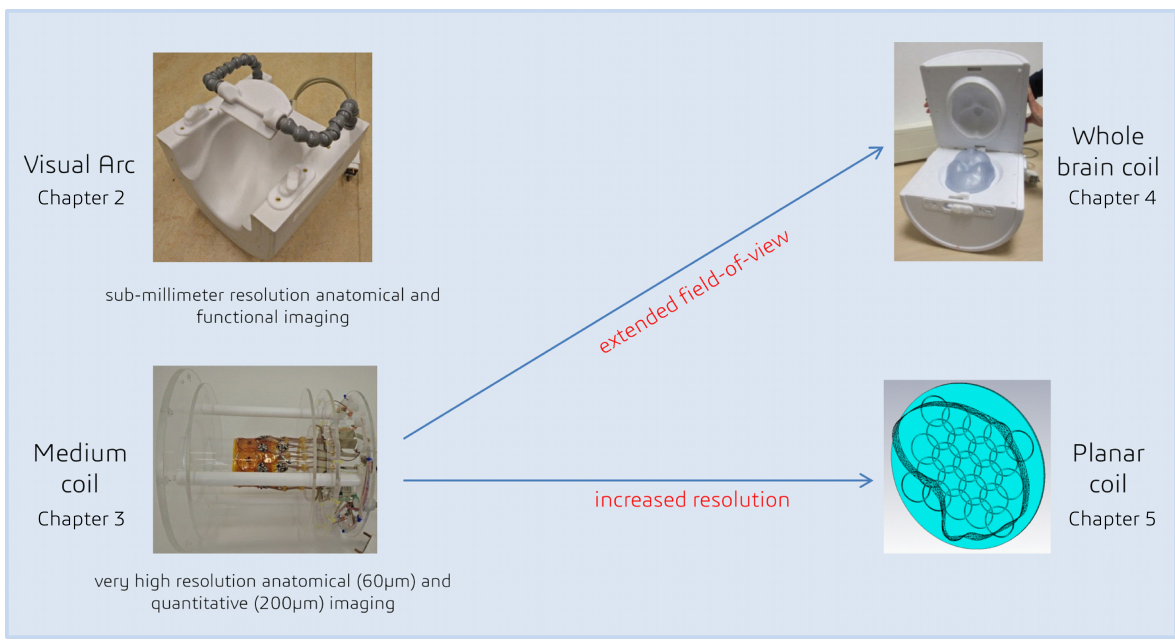

Figure 1.8: Overview of this thesis with details of coil development for localised, high resolution and large field of view MR imaging

The aim of this thesis is to investigate and develop RF coils for high resolution imaging of the human visual cortex at field strengths of 7T and above, with a focus of conformal, phased array coil geometries towards realising homogeneous $B_{1}$ transmit and receive fields and high Signal to Noise ratios (SNR). Novel RF coil layouts and designs are simulated, implemented and tested through the use of optimised RF pulse sequences, see Fig 1.8. The potential of conducting high resolution anatomical, functional and quantitative imaging are discussed, with an emphasis on functional and structural imaging of human cortical layers.

For imaging human cortical layer function, the main MRI tool is functional MRI (fMRI). fMRI acquisitions measure the brain activity through the detection of haemodynamic responses associated with stimuli [56, 57, 58]. MRI acquisition methods have had a substantial effect on our understanding of one of the most fundamental human sensory systems, namely the visual system and by extension, the hu- 
man visual cortex. Furthermore, cortical layer structure can also be investigated through several MRI contrast mechanisms $\left(T_{1}, T_{2}, T_{2}^{*}\right)$ to delineate cortical areas [59], allowing for so-called in-vivo histological MRI [60, 61]. However, the available resolution for in-vivo histological MRI remains insufficient to resolve individual cortical layers.

Therefore, with respect to imaging cortical layer structure, high resolution ex-vivo MRI can be used as the first step towards investigating MRI contrast in the cortex, which can then be validated by using established histology techniques on the same tissue. This is why after investigating state-of-the-art RF coil designs for in-vivo human cortical fMRI (Chapter 2), this thesis turns to coil designs for improved ex-vivo human cortical structure studies (Chapter 3-5).

In Chapter 2, a 16 channel receive coil array is paired with a 4 channel transmit coil array for high resolution functional and anatomical imaging of the human visual cortex at 7T. Compared to a whole head coil at 7T, we show that the designed coil provides a more homogeneous transmit field in the region of interest, upto 1.5 times more SNR, lower g-factors for accelerated imaging and is also capable of showing activation deeper into the occipital lobe.

In Chapter 3, we investigate the construction of a 16 channel cylindrical phased array receive coil for imaging large, post-mortem occipital lobe samples in a wide-bore 9.4T human scanner. Compared to the use of a whole head coil at 9.4T, the constructed coil showed SNR gains of up to 5 times, with increased transmit homogeneity achieved through parallel transmit techniques. Using this setup, we achieved $60 \mu \mathrm{m}$ anatomical images for the entire occipital lobe showing increased spatial definition of cortical details compared to lower resolutions, while also achieving sufficient control over SNR, $B_{0}$ and $B_{1}$ homogeneity and multi-contrast sampling to perform quantitative $T_{2}{ }^{*}$ mapping over the same volume at $200 \mu \mathrm{m}$ 
In order to facilitate extended field-of-view imaging of large, intact post-mortem brain samples, the design and characterisation of a conformal 24 channel receive / 8 channel transmit coil at 9.4T is detailed in Chapter 4. Given that $B_{1}$ field inhomogeneities tend to be particularly amplified at high field strengths, a combination of kT-points enabled parallel transmit methods, close-fitting receive coils and multicontrast sampling were used for generating anatomical and quantitative datasets at $100 \mu \mathrm{m}$ and $200 \mu \mathrm{m}$ respectively, with the aim of enabling brain atlassing and cortical laminar discrimination over the entire post-mortem brain.

Most ex-vivo histological MRI so far has been performed on thin slices of tissue (in the order of $\mu \mathrm{m}$ ) using bespoke RF coils in either a horizontal, high field animal scanner or in vertical bore scanner. In Chapter 5, we present a prototype of a large field-of-view, planar phased array receive and transmit coil capable of high resolution imaging of ex-vivo tissues slabs upto $17 \mathrm{~cm}$ in diameter and 5-10 $\mathrm{mm}$ in thickness. Given recent advances in histological staining and microscopy of thick post-mortem samples, this RF coil study presents a novel method of undertaking high resolution post-mortem imaging for subsequent coregistration with histological data.

Chapter 6 provides a brief summary and discussion of the work undertaken. 


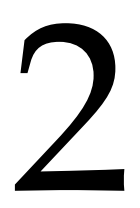

\section{A specialized multi-transmit head coil for high resolution fMRI of the human visual cortex at 7T}

\section{Contents}

2.1 Introduction . . . . . . . . . . . . . 33

2.2 Methods . . . . . . . . . . . . . . . . . . . . 35

2.3 Results . . . . . . . . . . . . . . . . . 46

2.4 Discussion $\ldots \ldots \ldots \ldots \ldots \ldots$

Adapted from: Sengupta S., Roebroeck A., Kemper V. G., Poser B. A., Zimmermann J., Goebel R., and Adriany G. A specialized multi-transmit head coil for high resolution $\mathrm{fMRI}$ of the human visual cortex at 7T. PLOS ONE, 11(12):1-22, 2016 


\section{Abstract}

\section{Purpose}

To design, construct and validate radiofrequency $(\mathrm{RF})$ transmit and receive phased array coils for high-resolution visual cortex imaging at 7 Tesla.

\section{Methods}

A 4 channel transmit and 16 channel receive array was constructed on a conformal polycarbonate former. Transmit field efficiency and homogeneity were simulated and validated, along with the Specific Absorption Rate, using $B_{1}^{+}$mapping techniques and electromagnetic simulations. Receiver signal-to-noise ratio (SNR), temporal SNR (tSNR) across EPI time series, g-factors for accelerated imaging and noise correlations were evaluated and compared with a commercial 32 channel whole head coil. The performance of the coil was further evaluated with human subjects through functional MRI (fMRI) studies at standard and submillimeter resolutions of up to $0.8 \mathrm{~mm}$ isotropic.

\section{Results}

The transmit and receive sections were characterized using bench tests and showed good interelement decoupling, preamplifier decoupling and sample loading. SNR for the 16 channel coil was $\sim 1.5$ times that of the commercial coil in the human occipital lobe, and showed better g-factor values for accelerated imaging. fMRI tests conducted showed better response to Blood Oxygen Level Dependent (BOLD) activation, at resolutions of $1.2 \mathrm{~mm}$ and $0.8 \mathrm{~mm}$ isotropic. 


\section{Conclusion}

The 4 channel phased array transmit coil provides homogeneous excitation across the visual cortex, which, in combination with the dual row 16 channel receive array, makes for a valuable research tool for high resolution anatomical and functional imaging of the visual cortex at 7T. 


\subsection{Introduction}

At clinical MR field strengths up to 3T, head optimized Radio Frequency (RF) coils tend to be receive-only designs which utilize larger RF body coils hidden behind the bore liner for homogeneous transmit field generation. This setup typically allows for excellent accessibility and space in the system bore, e.g. for fMRI task presentation capability. At ultra high fields, however, the RF wavelength nears the size of the object and RF penetration and interference patterns become a dominant concern [9]. This leads to non uniform $B_{1}$ field patterns and a significant difference between transmit and receive $B_{1}$ field profiles [33, 34, 25]. In this regime, standard RF body coil technology based on either quadrature birdcage or transverse electromagnetic (TEM) designs fail to achieve the required homogeneity and transmit efficiency, and thus RF transmit body coils are not implemented in commercially available Ultra High Field (UHF) scanners. Consequently at 7T it is required to either build a dedicated larger RF transmit coil and combine this with a receiver only array [63, 64, 65, 66] or to utilize transceiver arrays [23, 67, 68].

Functional images of the human brain at ultra-high fields of 7T and above are often acquired using a cylindrical quadrature volume transmit (Tx) coil and whole head phased array receive coil. Though such a fixed phase coil allows for a high $B_{1}^{+}$value at the center of the head, the Tx efficiency is significantly lower along the periphery and inferior extents of the brain, particularly along the occipital and inferotemporal visual cortex. Higher transmit efficiency can be achieved with transmit arrays which allow for greater $B_{1}^{+}$control through RF shimming methods. For fMRI applications that do not require whole head coverage and allow a more focused approach - such as studies of the visual and auditory cortex - it is possible to achieve very high resolution even with more limited number of receive coils [69]. Both these issues can be addressed by a) using a phased-array, semi-cylindrical Tx coil layout, where $B_{1}^{+}$can be homogenized by phase and amplitude shimming in the region of interest [70], and by b) additionally utilizing overlapping 
arrays of small receive coils with closely attached preamplifiers at 3T [50] and 7T [71].

For fMRI applications, cylindrical transmit coils close to the head significantly limit the ability for task presentation even in a $(60 \mathrm{~cm}$ diameter) body gradient setup. Task presentation is even more difficult if high performance head gradient inserts are used for higher spatial and temporal resolution imaging and the available space for coil housing and task presentation hardware decreases to around $36 \mathrm{~cm}$. For human visual system fMRI studies, both in the body as well head gradient setting, coil housing designs which are open at the anterior patient side have significant advantages in terms of fMRI task presentation ability as they easily allow both goggles or mirrored projection setups with large field of view. This is particularly important for ultra-high resolution studies of the visual cortex where both MRI data quality and stimulus presentation quality are of the utmost importance.

If transmit arrays are used in addition to the multiple receive elements, the phase and magnitude of the individual transmit elements can be adjusted (i.e., $B_{1}$ shimming) to provide an optimized transmit field. The optimized transmit field might entail uniformity throughout the brain, or it might be adjusted to provide maximum efficiency in a more targeted region of interest. Transmit arrays can mitigate Specific Absorption Rate (SAR) issues at high fields [72], as they can be designed to achieve maximum efficiency for the limited regions of interest and can also capitalize on $B_{1}$ shimming techniques.

On the receive side, because coil sensitivity profiles are used for spatial encoding, it is important for the receiver layout to comprehensively cover the volume under investigation. Earlier work in phased-array receiver coil design established the benefits of using close-fitting, small diameter surface coils for image locations close to the coil surface [21]. Wald and others [73, 74, 75, 48] showed that by incorporating denser receiver coil matrices over the same total surface area, the sensitivity profile of the coil array could be increased substantially for locations 
close to the coil array surface, ideally without compromising sensitivity at larger distances into the imaging volume. Their analysis predicts that with the increase in the number of receiver channels, it is possible to increase the sensitivity near the array without compromising or losing the sensitivity at distances further away from the coil plane[50].

Here we evaluate the advantages of building an open, half cylindrical Tx coil layout with a conformal small loop size receive $(\mathrm{Rx})$ coil layout optimized for functional MRI of the occipital and inferior and middle temporal visual cortex at high spatial resolution and compare this with a standard whole head array. For the initial half volume coil work we limited ourselves to a 16 receive channel layout for a fairer comparison to the 32 channel whole head array. The coil geometry is chosen such that the receiver coils maintain the critical overlap between neighbouring coil elements, thus minimizing mutual inductance while covering the entire occipito-temporal visual cortex, with very high SNR in the cortex. The coils are mounted on a close-fitting former [76] to maintain close proximity between subject and the receive array. For maximal image SNR, acceleration, SAR reduction, and subject access (i.e. visual presentation, motion monitoring cameras or motion reducing bite bars) an open coil as well as a combination of transmit and receive arrays is desired.

\subsection{Methods}

\section{Coil design}

The coil was constructed on a 3D printed former made from polycarbonate (PC) using the Fused Deposition Modelling (FDM) (Virtumed LLC, Minneapolis, MN, USA ) method and consists of 2 modular parts: one for dedicated receive and one for dedicated transmit function. 
16 receive coils were built on a conformal former that was modeled after an average-sized human head (Fig 2.1A). The inner receive array former is $3 \mathrm{~mm}$ thick to ensure minimal distance between the coil and the anatomies under interest, thus maximizing higher receive signal sensitivity. The receive coil layout was designed to ensure maximum SNR in the visual cortex, extending from early visual areas (V1-V4) anterior into occipital, inferior and lateral temporal cortex. Given that the 2 rows of receive coils encapsulate the region of interest in an arc, the coil was named as "Visual Arc". The receive coils were constructed using $2 \mathrm{~mm}$ thick silver-coated copper wires - with each individual coil being $6 \mathrm{~cm}$ in diameter - and are laid out in a $8 \times 2$ matrix of 16 coils that encapsulate the human occipito-temporal visual cortex (Fig 2.1C, 2.2B). A critical overlap (of 0.20 to 0.25 times the loop diameter of each coil; curvature of the receive former dictated the optimal overlap in case of particular coil elements)[21] between all coils was maintained to minimize mutual inductance between coils and to ensure mutual decoupling. Teflon tubing was inserted over each coil such that the overlapping areas of each coil pair would be sufficiently insulated from each other. Two distributed capacitors for each circular loop were divided symmetrically along the coil loop. A detailed schematic of the individual receive coil element is shown in Fig 2.3.

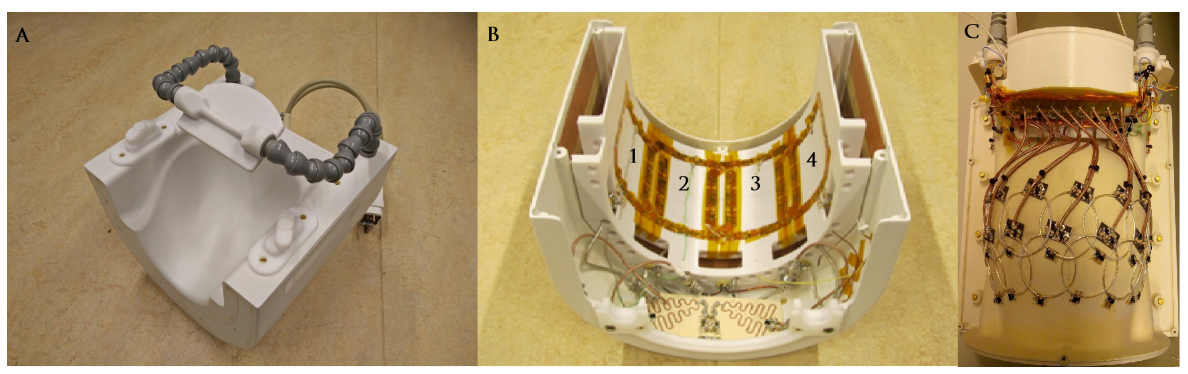

Figure 2.1: 7 Tesla close fitting dedicated visual cortex coil for high resolution imaging. (A) Shows the assembled coil with provisions for wide fieldof-view task presentation mirror (B) The 4 channel phased-array transmit coil with a dedicated power splitter (C) Shows the $2 \times 8$ receive coil layout, cable routing and the preamplifier box. 


\section{A}

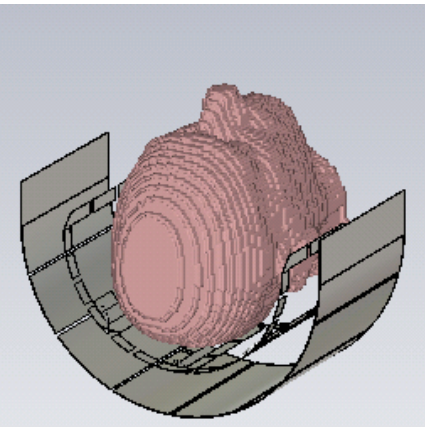

B

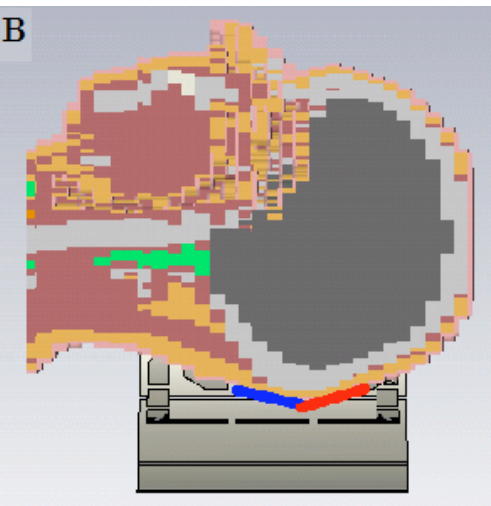

Figure 2.2: Transmit and receive setup. (A) Design of the 4-channel phased array transmit coil with an RF shield, showing the placement of the subject relative to the coil. (B) The 2 receiver coil rows, indicated in blue and red, encapsulate the human visual cortex.

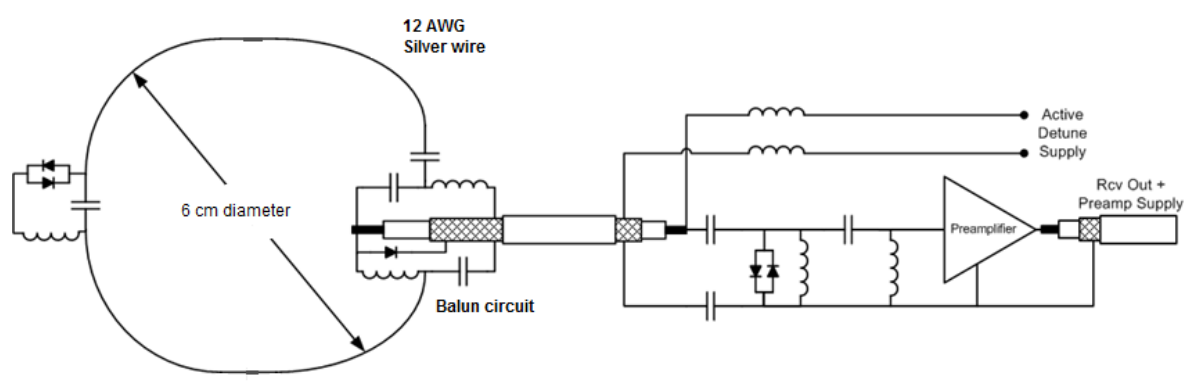

Figure 2.3: Receive coil schematic. Design of a single receiver coil with the balun circuit, split capacitors, passive and active detuning and preamplifier attached with a $\lambda / 4$ coaxial cable

A 4-channel half-circle phased array transmit coil was constructed concentric to the phased array receive circuit using $1.2 \mathrm{~cm}$ wide adhesive copper tape (3M, Minnesota, USA) on a half-cylindrical, 3D printed former (Stratasys, Eden Prairie, MN, USA). Fig 2.2 shows a representation of the placement of the subject, relative to the transmit array and 
also the placement of the 2 rows of receive coils and their proximity of the sample under investigation.

\section{Coil circuitry}

Each receive coil was connected to a lattice-balun or LC balun circuit [48],[77] and a tuning and matching network comprising of highvoltage ceramic trimmer capacitors $C_{T}$ and $C_{M}$ (1-10pf, Johanson Technology, CA, USA) for tuning the coil to resonance at 7 Tesla and matching the coil's output impedance to a noise match of $50 \Omega$. Using small FR4 boards, passive detuning circuitry consisting of an LC circuit in parallel with a high voltage diode was placed on the loop, opposite to the balun circuit. Each coil was tuned and matched to $297.2 \mathrm{MHz}$ and connected to a low input-impedance preamplifier (WMA7TRA, WanTcom Inc., MN, USA) using $\sim \lambda / 4(170 \mathrm{~mm})$ length coaxial cable (Huber-Suhner, K02252D), to achieve preamplifer decoupling between individual coil elements by transforming the high impedance at the coil output to the corresponding low impedance at the preamplifier input [21],[77]. The balun circuit incorporates an active detuning circuit across the matching capacitor, using the balun inductor and a PIN diode. When forward biased, the parallel resonant LC circuit adds a high impedance in series with the coil loop, effectively blocking current flow in the receive loop during transmit. The cable length was adjusted carefully for each coil to achieve preamplifier decoupling without requirement of further lumped element phase shifting circuitry. The cables of the lower row were then routed along the center of the upper row coil loops (virtual ground plane) such that there was minimal influence on the reflection and transmission parameters of two adjacent elements in each row.

The preamplifiers themselves were arranged in a $8 \times 2$ matrix mirroring the receive coil layout and are placed on a "preamplifier motherboard" which contained the required circuitry for providing the PIN 
diode voltages for actively detuning each receive coil element during transmit. The preamplifiers were also arranged along the $B_{0}$ field or the z-direction of the magnet to minimize any Hall effect which might affect the field effect transistors (FETs) used in these preamplifiers [78]. Cable traps on the output of each preamplifier were required and implemented - by fashioning a solenoid ( 2 turns, $4 \mathrm{~mm}$ diameter) from the coaxial cable connecting the preamplifier output to the plug that connects to the magnet bed and bridging a variable capacitor across its ends and tuning it to the resonant frequency of 297.2 MHz. Neighboring cable traps were oriented perpendicular to avoid interactions. The traps were all positioned outside the immediate RF transmit loop locations. The traps significantly reduced common-mode currents and were found to be essential to suppress interactions with the transmit coil.

Of the 4 coil elements of the transmit array, the two peripheral coils are $12 \times 12 \mathrm{~cm}^{2}$ and contain ten $12 \mathrm{pf}$ capacitors distributed along the coil length, while the two central coils are $12 \times 14 \mathrm{~cm}^{2}$ with four $12 \mathrm{pf}$ and six 10pf distributed along the coil length, thus ensuring an extended coverage of the occipital pole and cerebellum (Fig 2.1B, 2.2A). A Wilkinson power splitter was implemented at the transmit input to split the transmit signal into 4 equal amplitude excitations for each transmit coil. Each loop was initially phase-shifted by $45^{\circ}$ relative to the previous loop by using cables of differing phase lengths, thus ensuring a $135^{\circ}$ phase shift between the farthest transmit elements. An RF shield was placed concentric to the transmit array, $3 \mathrm{~cm}$ away from it. The shield was designed from double sided $50 \mu \mathrm{m}$ thin copper sheet with 8 etched overlaps to minimize eddy current losses. Each transmit loop was tuned and matched to $297.2 \mathrm{MHz}$ and $50 \Omega$ respectively using high-voltage variable capacitors (NMNT10-6E, Voltronics Corp, MD, USA), with high-power PIN diodes (MA4PK3000, Macom, MA, USA) connected to each coil in order to detune the transmit array during receive. (Fig 2.4) 


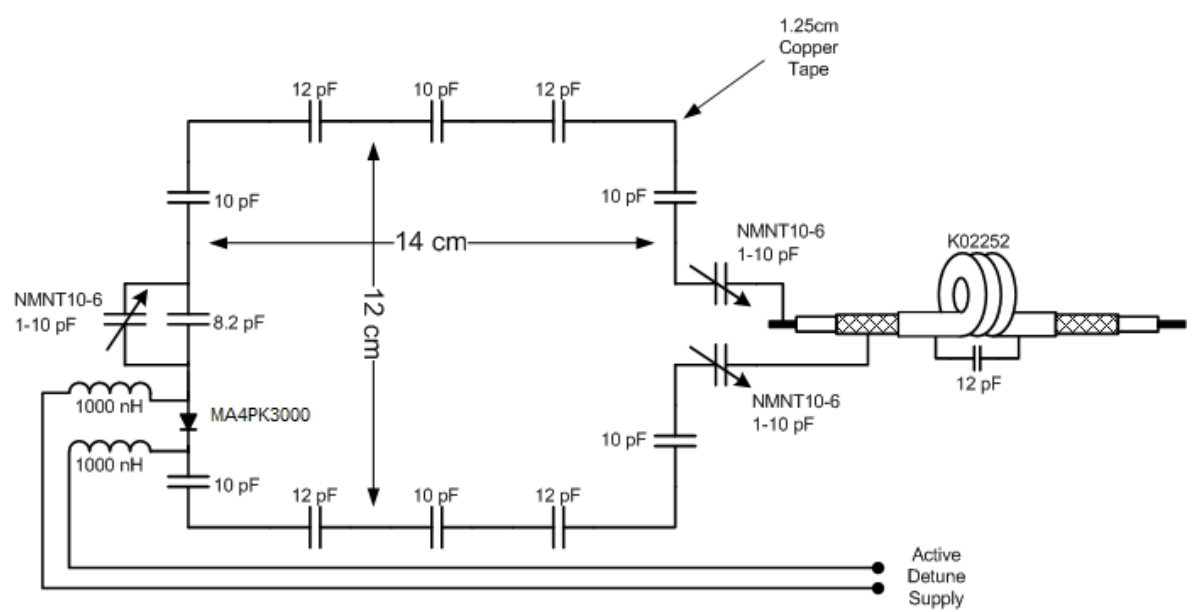

Figure 2.4: Transmit coil schematic. Design of a single transmit coil with distributed capacitors, tuning and matching capacitors and active detuning.

\section{Bench measurements}

Bench tests were performed using an Agilent HP E5071C ENA Series network analyzer, a customized coil-plug bed and a human torsoshaped phantom (49.8\% demineralized water, $48.8 \%$ sucrose, $1.3 \%$ $\mathrm{KCl}, 0.10 \%$ Dowicil; Max Planck Institute for Biological Cybernetics, Tübingen, Germany ) as load. Measurements were performed to check for loaded $\left(Q_{L}\right)$ and unloaded $\left(Q_{U}\right) \mathrm{Q}$ ratios, receive coil decoupling and preamplifier decoupling for each coil element. The measurements on the bench validated coil tuning and matching, preamplifier decoupling for each coil element and active coil detuning.

The coil quality factor ratio $\left(Q_{U} / Q_{L}\right)$ was measured for a single receive coil element using a dual-loop decoupled $(\sim 50 \mathrm{~dB})$ inductive probe lightly coupled to the coil loop under test: once as a single element outside the receiver array and once within the populated array while keeping all other receive elements in a detuned state. 
Each loop on the receive array was tuned to the corresponding Larmor frequency of $297.2 \mathrm{MHz}$ and matched to $50 \Omega$. Coupling between neighbouring elements was additionally measured through $S_{21}$ measurements by directly connecting the coil outputs to the network analyser, while keeping all other elements detuned. Using this setup, the overlap between neighbouring elements could be further optimized to ensure minimal mutual inductance.

Preamplifier decoupling of a single loop was measured as the change in the $S_{21}$ measurement using a pair of deoupled pickup probes, when the coil element was first power matched to $50 \Omega$ under load but without the preamplifier present and when the coil was terminated using the powered-on low input impedance preamplifier, while all other coils in the receive array were detuned. The active detuning for each receive element was measured as the difference in an $S_{21}$ measurement, using a pair of decoupled pickup probes, between when the coil is matched to $50 \Omega$ and when detuned.

The assessment of the characteristics of the 4-channel transmit loop was undertaken in the same manner. Each loop of the transmit channel was tuned and matched to $297.2 \mathrm{MHz}$ and $50 \Omega$ respectively using an $S_{11}$ measurement, with the coil connected directly to the network analyser while loaded with the human-torso phantom. The geometric coupling between adjacent coil elements was measured as the $S_{21}$ between the outputs of the loaded coil. Active detuning of each coil was recorded as an $S_{21}$ measurement using a pair of decoupled pickup probes lightly coupled to the coil under investigation, and forward biasing the diode in order to detune the coil. Each coil was terminated to $50 \Omega$ during this measurement, while keeping the other 3 loops in the detuned state. 


\section{EM Coil simulation}

The 4 - loop phased array transmit coil was simulated in CST (Computer Simulation Technologies, Darmstadt, Germany) in the timedomain, to determine the excited $B_{1}^{+}$field strength and homogeneity, and to also validate the coil for Specific Absorption Rate (SAR) limits [79]. The coil elements were defined using the software's in-built 3D CAD designer and included all 4 transmit elements as perfect electrical conductors, with 2 discrete and 8 lumped element ports on each transmit coil.

The coil elements were all precisely modeled to accurately reflect the physical design of the coil, including the dimensions and material properties of the elements. The $2 \mathrm{~mm}$ diameter wire used for connecting overlapping segments of each coil was also modeled into the simulation setup. The discrete ports allowed us to place variable tuning / matching capacitors and $50 \Omega$ ports in the simulated coil schematic along with predefined capacitors (RLC, series) placed at the lumped element ports along each coil.

The simulation paradigm was completed by placing a human voxel model extended up to the shoulders (Gustav, HUGO voxel family, CST, $2 \mathrm{~mm}$ isotropic resolution) as the load for the transmit coil and defining a high-resolution mesh ( $>50$ million mesh cells) around the coil and load. The receive array, along with cable traps, coaxial cable lines and PIN diode detuning cable lines were excluded from the simulation to avoid complex 3D meshing and to save processing time. SAR maps were generated using the electric and magnetic field monitors available through the software and were averaged to $1 \mathrm{~g}$ and $10 \mathrm{~g}$ volumes over the voxel model's region of interest. The calculated SAR profiles are shown in Fig 2.5. 


\section{MRI Data Acquisition and Reconstruction}

Data was acquired on a Siemens MAGNETOM 7 Tesla activelyshielded MRI system (SIEMENS Healthcare, Erlangen, Germany) with an SC72 gradient coil capable of a maximum gradient strength of $70 \mathrm{mT} / \mathrm{m}$ with a maximum slew rate of $200 \mathrm{~T} / \mathrm{m} / \mathrm{s}$. Data pertaining to coil characterization, namely tSNR, g-factor maps and noise correlation matrices were obtained through in-vivo scans across both the Visual Arc and a 32 channel whole head coil (Nova Medical Inc., MA, USA) for two healthy subjects. Subjects for the study filled out a written consent form prior to imaging, as approved by the Ethical Review Committee Psychology and Neuroscience (ERCPN) of Maastricht University.

Transmit field or $B_{1}^{+}$field quantification was achieved by using the Actual Flip Angle method (AFI) [80]. In-vivo tSNR (Temporal Signal-toNoise Ratio) maps were obtained at two different resolutions using the University of Minnesota's multi-band EPI package for Gradient Echo BOLD EPI [81] with the following parameters: Echo Time (TE) $=17 \mathrm{~ms}$; Repetition Time (TR) = 2000 ms; Partial Fourier $(P F)=6 / 8$; GRAPPA 3; Bandwidth $(\mathrm{BW})=1488 \mathrm{~Hz} / \mathrm{Px}$; Echo spacing $=0.78 \mathrm{~ms}$; field of view $(\mathrm{FoV})=192 \times 192 \mathrm{~mm}$ for a $1.2 \mathrm{~mm}$ isotropic acquisition and Echo Time $(\mathrm{TE})=23 \mathrm{~ms}$; Repetition Time $(\mathrm{TR})=2000 \mathrm{~ms}$; Partial Fourier $(\mathrm{PF})=$ 6/8; GRAPPA 3; Bandwidth $(B W)=1102 \mathrm{~Hz} /$ Px; Echo spacing $=1.03$ $\mathrm{ms} ; \mathrm{FoV}=130 \times 130 \mathrm{~mm}$ for a $0.8 \mathrm{~mm}$ isotropic acquisition. Noise covariance data was acquired using the same pulse sequence with the transmit voltage set to 0 volts, or without an RF excitation.

For phantom datasets the following parameters were used for EPI acquisitions: Echo Time (TE) = $18 \mathrm{~ms}$; Repetition Time $(\mathrm{TR})=3000 \mathrm{~ms}$; Partial Fourier $(\mathrm{PF})=6 / 8$; GRAPPA 3; Bandwidth $(\mathrm{BW})=1394 \mathrm{~Hz} / \mathrm{Px}$; Echo spacing $=0.82 \mathrm{~ms}$; field of view $(\mathrm{FoV})=166 \times 166 \mathrm{~mm}^{2}$ for a $1.2 \mathrm{~mm}$ isotropic acquisition and Echo Time $(\mathrm{TE})=27.6 \mathrm{~ms}$; Repetition Time $(\mathrm{TR})=3000 \mathrm{~ms}$; Partial Fourier $(\mathrm{PF})=6 / 8$; GRAPPA 3; Band- 
Chapter 2. A specialized multi-transmit head coil for high resolution fMRI of the human visual cortex at 7T

width $(\mathrm{BW})=1002 \mathrm{~Hz} / \mathrm{Px}$; Echo spacing = $1.1 \mathrm{~ms} ; \mathrm{FoV}=166 \mathrm{X} 166$ $\mathrm{mm}^{2}$ for a $0.8 \mathrm{~mm}$ isotropic acquisition.

Image SNR for both coils was computed using a pseudo-replica approach [82, 83] from the short-TE GRE image and noise data acquired using the same subject, which is also the data used for g-factor analysis. Noise data was acquired using each coil without RF excitation and the standard deviation and correlation of the noise data were computed. We generated a 100 replicas by adding random samples from the noise scan onto the GRE k-space of the standard image acquisition prior to Fast Fourier Transform (FFT), while maintaining the noise correlation between the receive channels. The final image SNR was then calculated as the ratio of the mean of the image to the standard deviation of the noise over these 100 replicas. A mask covering the brain volume containing the occipital lobe was created to determine SNR values.

tSNR maps were computed as the mean signal intensity over time divided by the standard deviation of the time series, averaged over a 100 volumes after detrending for linear and 1-2 cycle oscillations and up to second order harmonic oscillations. Data analysis was performed using BrainVoyager QX 2.8 (Brain Innovation, Maastricht, The Netherlands) and custom-written routines in MATLAB (The MATHWORKS Inc., Natick, MA, USA). In addition to the MPRAGE and and fMRI scans, we acquired noise-only scans and fully sampled 3D GRE for evaluation of the noise correlation and the parallel imaging performance of the two coils. Both scans (in-vivo and phantom) were performed using a 3D GRE sequence with the following matched parameters: Echo Time $(\mathrm{TE})=2.64 \mathrm{~ms}$; Repetition Time $(\mathrm{TR})=30 \mathrm{~ms}$; FoV = $256 \times 256 \mathrm{~mm}^{2} ; 2 \mathrm{~mm}$ isotropic; the noise scan was acquired with fewer $K_{Y}$ and $K_{Z}$ encode and by turning the transmitter voltage to zero. The GRE scan was Fourier-interpolated to a matrix size of [120 $\times 120 \times 120]$ in order to allow emulation of different SENSE undersampling factors on the bases of data set $(R=2,3,4,5,6,8)$. For a quantitative comparison of the parallel imaging performance of the two coils, noise correlation matrices, coil sensitivity maps and g-maps were calculated in 
MATLAB.

Sensitivity maps were computed as described by Walsh [84]. For the $\mathrm{g}$-factor maps we used the formulation given in the original SENSE paper [18].

$$
g_{\rho}=\sqrt{\left[\left(S^{H} \Psi^{-1} S\right)^{-1}\right]_{\rho, \rho}\left(S^{H} \Psi^{-1} S\right)_{\rho, \rho}}
$$

where $\Psi$ is the covariance matrix. Both g-factors and corresponding SENSE reconstructions were performed at undersampling factors between 2 and 6 , by sub-sampling the full k-space data set that had been re-sampled to [ $120 \times 120 \times 120]$. The reconstruction itself was a SENSE reconstruction as described in [18]. We have chosen SENSE g-maps because we feel that this is more general, but alternatively GRAPPA or pseudo-replica based g-maps could have been shown.

The visual paradigm used for fMRI data acquisition consisted of high contrast flickering checkerboards (flicker rate of $7.5 \mathrm{~Hz}$ ) as a passive viewing task with central fixation dot on the screen. A block design was chosen with 18 blocks of checkerboards with length 4 TRs (8s) interspersed with rest periods of 5,6 , or 7 TRs (jittered). The frontalopen design of the 16 channel coil allowed for a visual angle was $29^{\circ}$ extending into the subjects' near-peripheral vision; for the 32 channel whole head coil the visual angle was $10^{\circ}$ - extending into the subjects' central and paracentral vision [58] - as its viewing angle is limited due to the coil's design. 


\subsection{Results}

Inter-element decoupling between pairs of transmit elements was $13 \mathrm{~dB}$ or lower. Active pin-diode detuning of each transmit loop, recorded as an $S_{21}$ measurement using a double-loop probe, was about $-20 \mathrm{~dB}$. Table 1 shows the S-parameter matrix for the 4-channel phased array transmit coil when loaded with the human-torso phantom. Electromagnetic simulations of transmit array for SAR validation yielded a peak SAR value of $0.385 \mathrm{~W} / \mathrm{kg}$ in the human voxel model for a fixedphase increment across coils, for a 1 Watt sinusoidal RF excitation split across 4 channels equally through the Wilkinson power splitter, as illustrated in Fig 2.5. To ensure patient safety, a conservative factor of $1 \mathrm{~W} / \mathrm{kg}$ was chosen as the SAR limit.

Table 2.1: S-parameters in $\mathrm{dB}$ showing the transmission and reflection coefficients for the 4-channel transmit coil when loaded with the human torso phantom.

\begin{tabular}{|c|c|c|c|c|}
\hline Channel & 1 & 2 & 3 & 4 \\
\hline 1 & -29.8 & -13.5 & -15.2 & -21.4 \\
\hline 2 & & -30.3 & -14.2 & -16.6 \\
\hline 3 & & & -28.2 & -14.8 \\
\hline 4 & & & & -31 \\
\hline
\end{tabular}




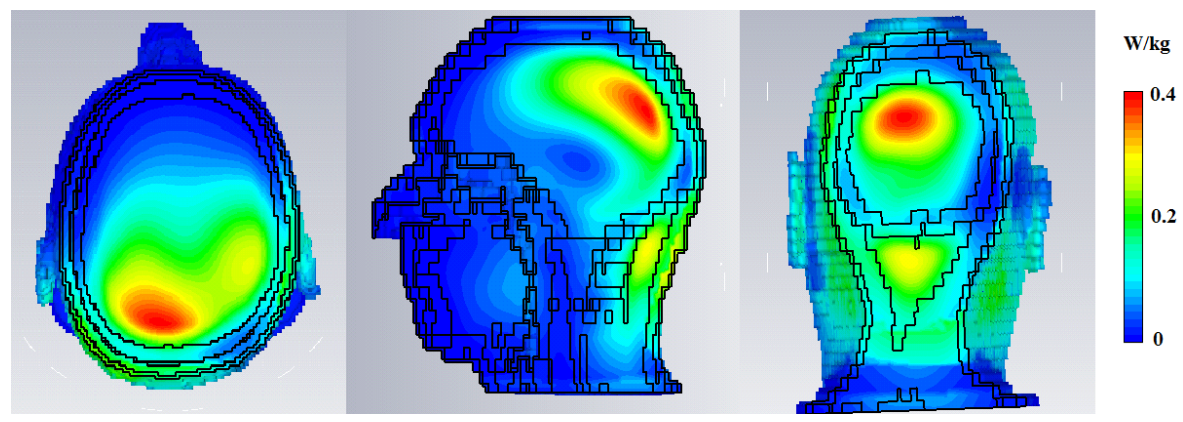

Figure 2.5: SAR results. Specific Absoprtion Rate or $\mathrm{SAR}_{10 g}$ for the HUGO voxel model as simulated in CST, shown along all 3 axes. Peak SAR $_{10 g}$ was computed as $0.385 \mathrm{~W} / \mathrm{kg}$.

Fig 2.6 shows the simulated and measured $B_{1}^{+}$maps along a transverse slice for the voxel model and the human subject respectively. The measured $B_{1}^{+}$data was acquired using a quantitative $B_{1}$ mapping method, co-registered to an anatomical image of the same slice in the axial plane. Both simulation and measured data show good correlation between each other, with a substantially homogeneous $B_{1}^{+}$excitation profile achieved across the visual cortex.

The unloaded $\mathrm{Q}$ factor $Q_{U}$ (indicative of coil losses) for an isolated receive element was about 240 and the loaded $Q$ factor $Q_{L}$, indicative of coil and tissue losses, was 25 , giving us a $Q_{U} / Q_{L}$ of about 9.6, indicating sample noise dominance. For a single receive loop surrounded by 5 detuned loops in the array, the same $Q$ factor measurement yielded a $Q_{U} / Q_{L}$ of $8.6(232 / 27)$. All receive coils were matched between $19 \mathrm{~dB}$ and $-23 \mathrm{~dB}$ for the human torso phantom and for up to 3 different subject-head sizes. The active PIN diode detuning provided an isolation better than $25 \mathrm{~dB}$ between tuned and detuned states. The decoupling between neighbouring, overlapping receive elements ranged between $-12 \mathrm{~dB}$ to $-18 \mathrm{~dB}$. Decoupling between next-nearest neighbouring elements (or non-overlapping elements) of the array ranged between $-15 \mathrm{~dB}$ to $-24 \mathrm{~dB}$. Preamplifier decoupling accounted for an additional $20 \mathrm{~dB}$ of isolation. The noise correlation matrix, as shown in Fig 2.7, 
Chapter 2. A specialized multi-transmit head coil for high resolution fMRI of the human visual cortex at $7 T$

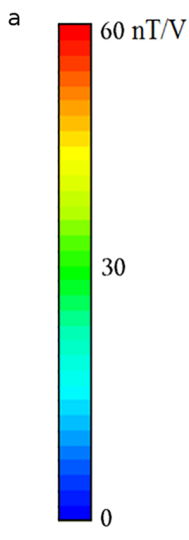

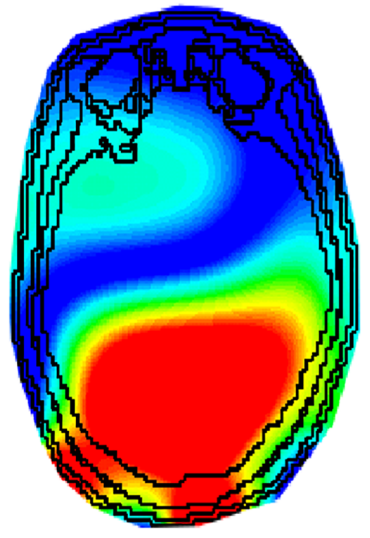

Simulation

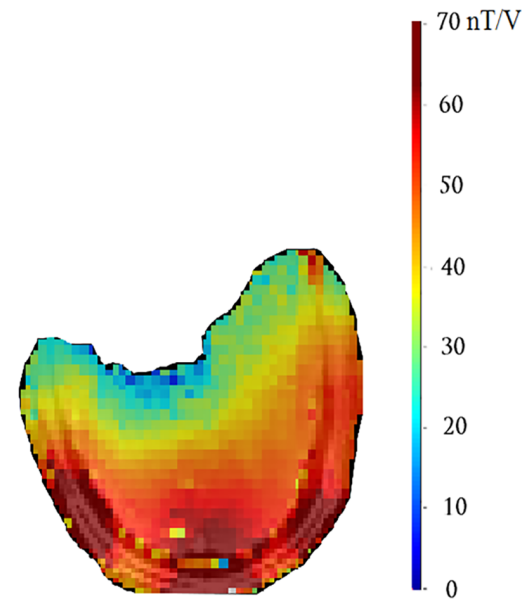

Measurement

b
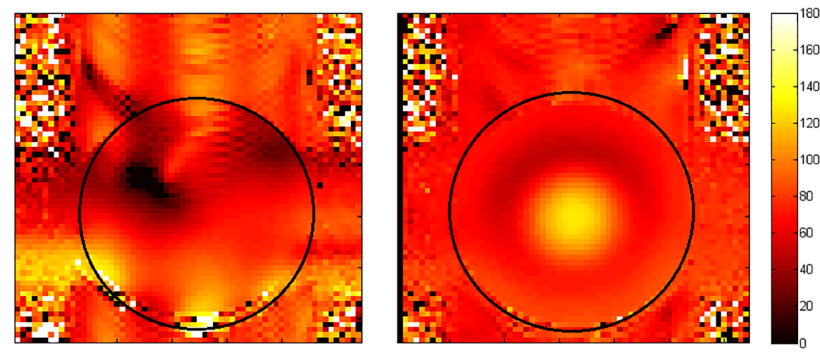

Figure 2.6: Transmit field maps. (a) Axial slices showing simulated (L) and measured (R) $B_{1}^{+}$field maps in nT/V. (b) Flip angle maps of the Visual Arc $(\mathrm{L})$ and Nova coils (R) using a spherical phantom represented by the black circle 


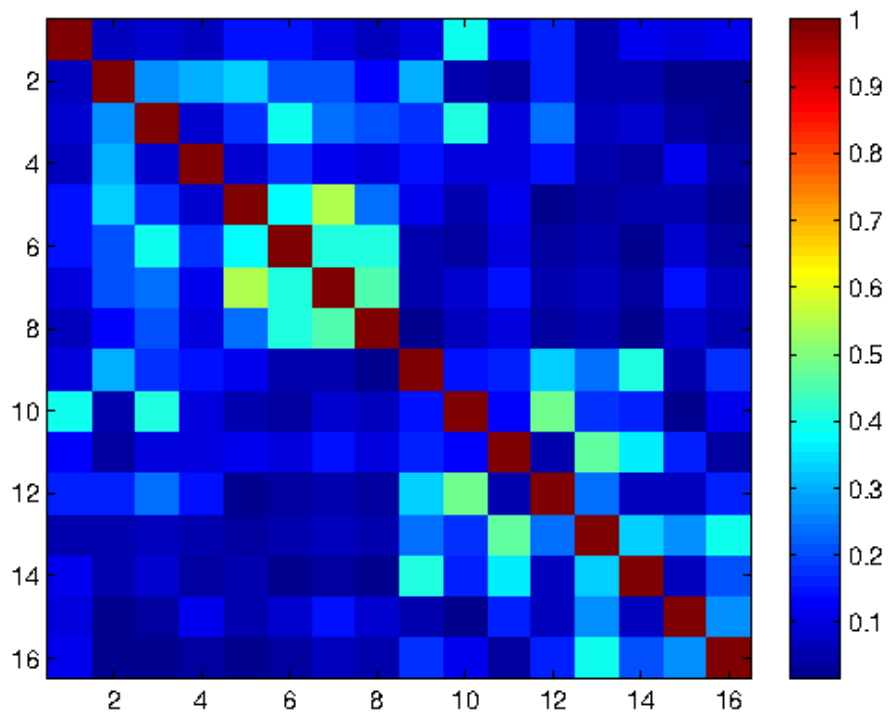

Figure 2.7: Noise correlation. Noise correlation matrix for the constructed 16 channel Visual Arc coil, with a peak of $54 \%$ and a mean of $19 \%$.

was obtained with in-vivo scanning, with the correlation ranging between $0.2 \%$ to $54 \%$ with an average of $19 \%$.

Image SNR for both coils is shown in Fig 2.8 for in-vivo scans and Fig 2.9 for a spherical phantom. The mean SNR in the masked volume was 5318 for the 32 channel whole brain coil and 7139 for the Visual Arc coil, while the maximum SNR values in the same volume were 6988 and 10014 for the whole head coil and the Visual Arc coil respectively.

Temporal SNR maps were generated for both the 16 channel visual coil and the standard 32 channel whole head coil at $1.2 \mathrm{~mm}$ isotropic resolution using a gradient echo - EPI sequence and the same measurements were repeated with a spherical phantom. A small region of interest covering the primary and secondary visual areas (V1 \& V2) was defined and maximum and mean tSNR values were computed in 
Chapter 2. A specialized multi-transmit head coil for high resolution fMRI of the human visual cortex at $7 T$
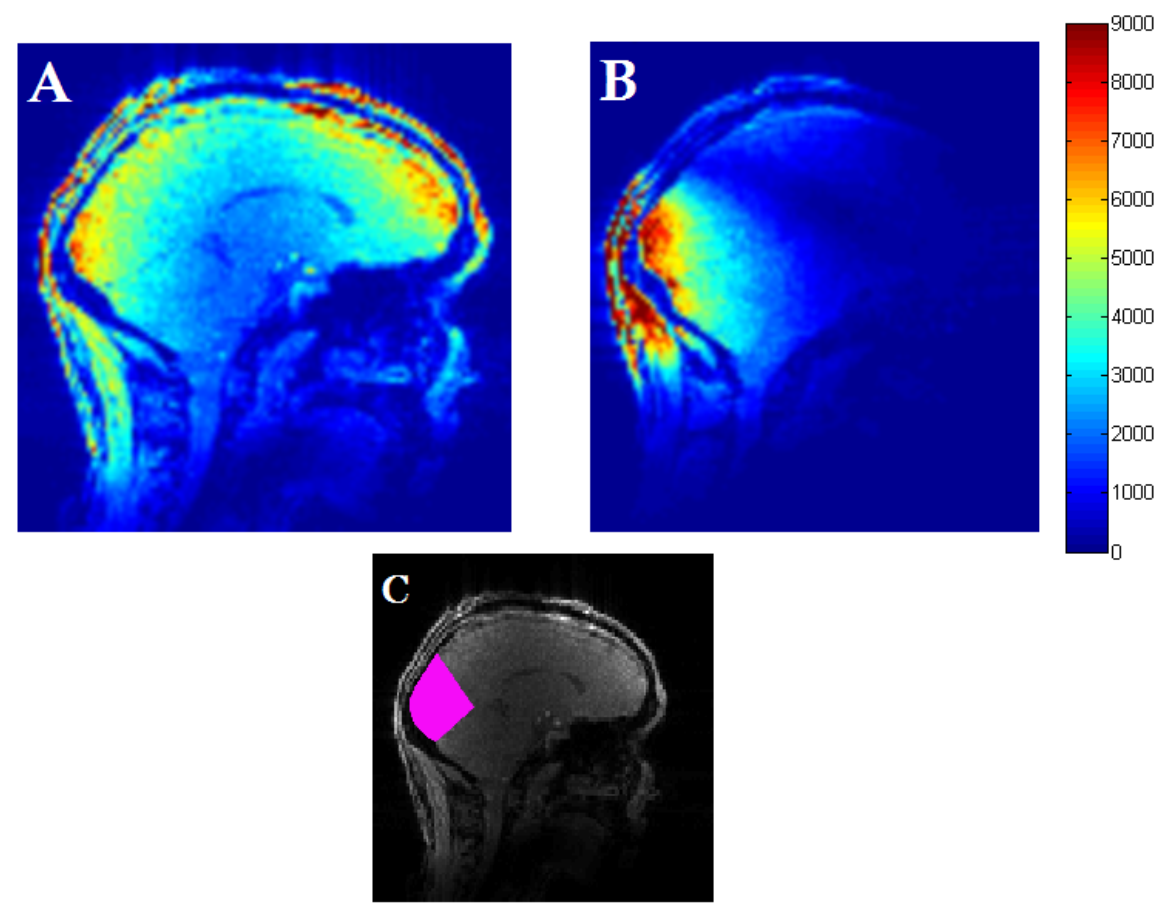

Figure 2.8: Signal-to-Noise comparison. (A) SNR map of the 32 channel head coil with a human subject (B) SNR map of the 16 channel Visual Arc coil, with the same colour scale used for both maps. (C) Mask (in pink) showing brain volume used for SNR calculation. 


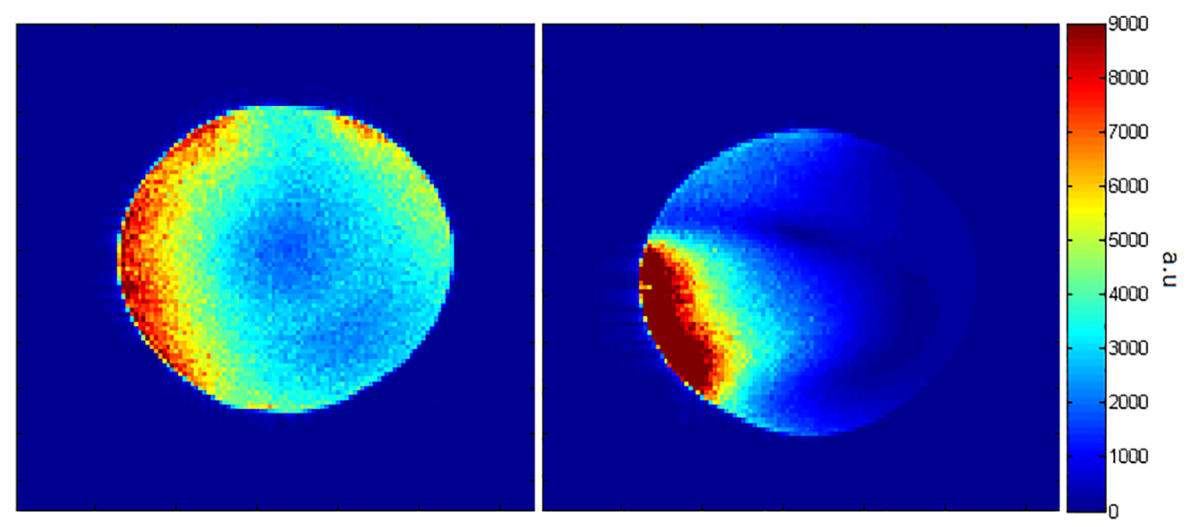

Figure 2.9: Signal-to-Noise comparison. (L) SNR map of the 32 channel head coil with a spherical phantom and (R) SNR map of the 16 channel Visual Arc coil, with the same colour scale used for both maps.

the same ROI. Fig 2.10 shows the maximum and mean $t S N R$ value estimates generated by both coils in the primary and secondary visual areas at $1.2 \mathrm{~mm}$ isotropic. tSNR was about 1.5 times higher with the 16 channel Visual Arc coil (than with the 32 channel whole head coil), with the high $\mathrm{tSNR}$ receive profile extending into the inferotemporal cortex. The receive array provides high SNR and sufficient penetration depth to image the visual cortex into sulcal depths.

Fig 2.11 shows the tSNR estimates for the same subject at $0.8 \mathrm{~mm}$ isotropic resolution. Fig 2.12 and Fig 2.13 shows the entire slice stack of the phantom EPI measurement, acquired using both left-right and anterior-posterior phase encoding. tSNR profiles along a central slice were plotted for both coils at 1.2 and $0.8 \mathrm{~mm}$ isotropic using a spherical phantom. Fig 2.14 shows the tSNR profiles as a function of distance from the edge of the phantom. 
Chapter 2. A specialized multi-transmit head coil for high resolution fMRI of the human visual cortex at 7T

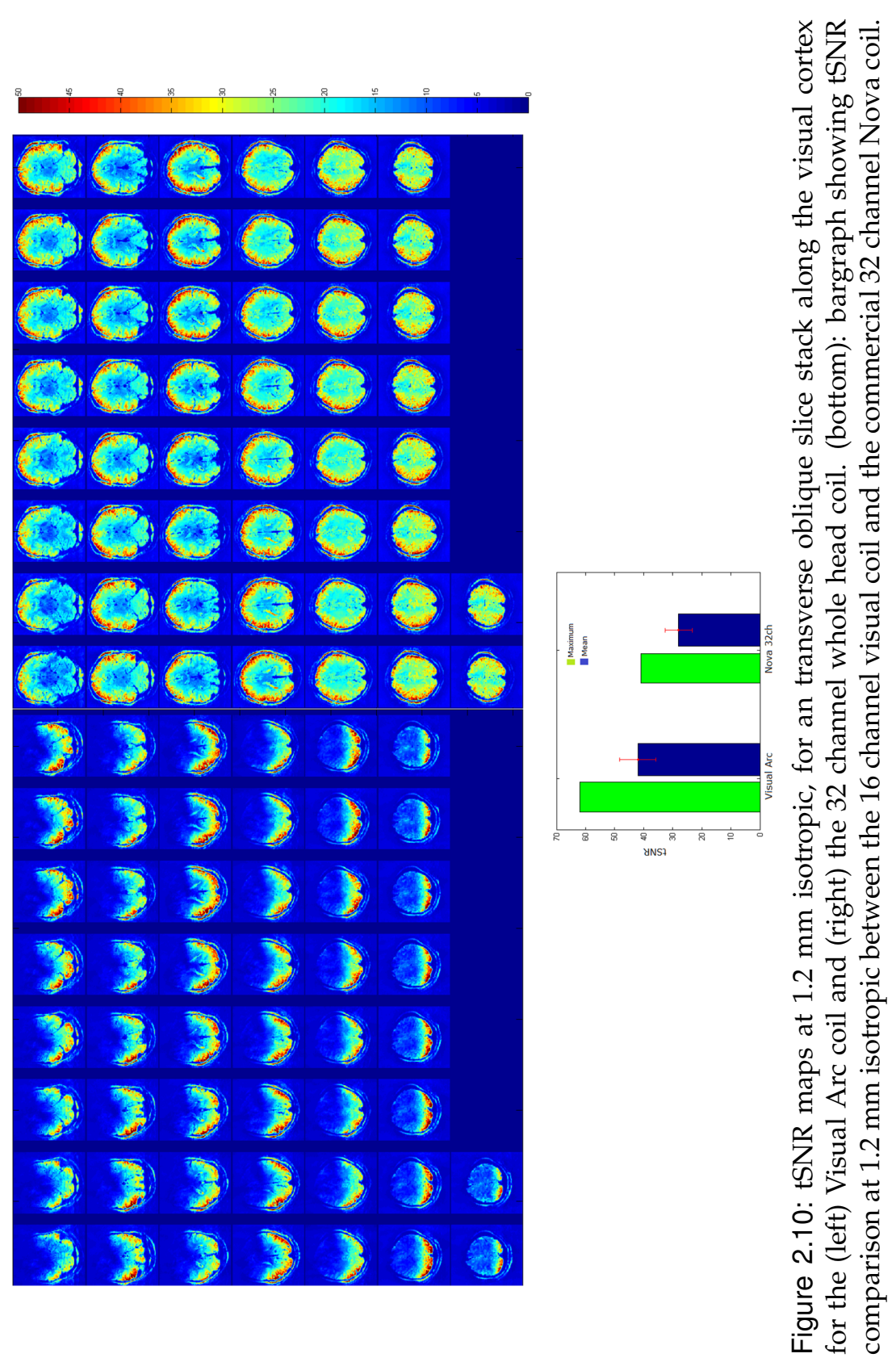




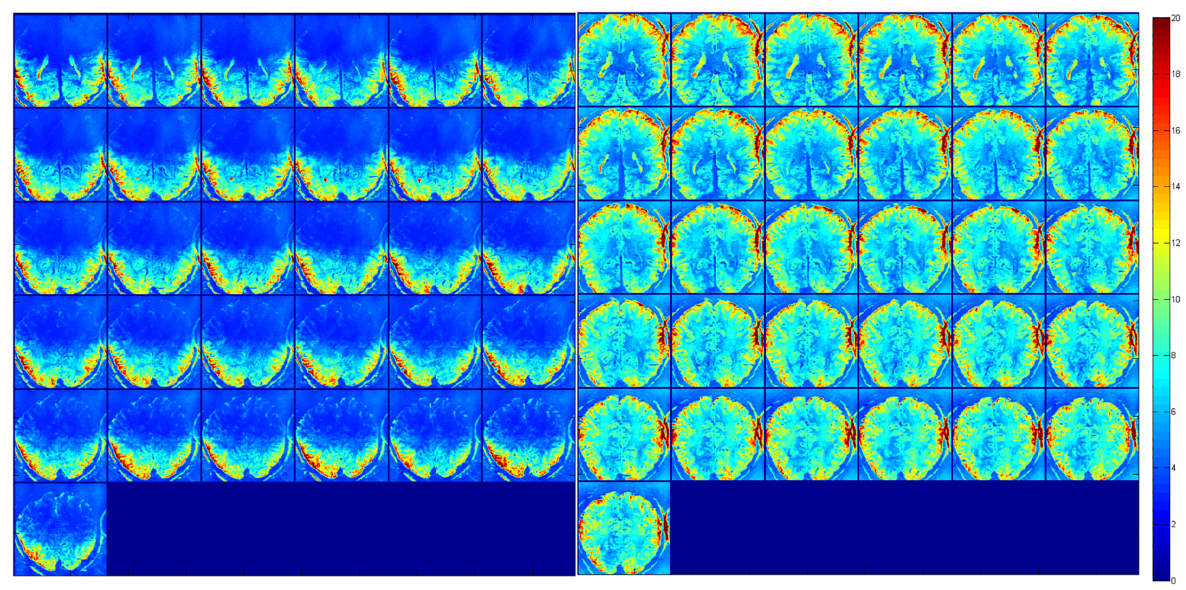

Figure 2.11: tSNR maps at $0.8 \mathrm{~mm}$ isotropic, for an transverse oblique slice stack along the visual cortex for the (left) Visual Arc coil and (right) the 32 channel whole head coil.

Local g-factor maps in a coronal plane were generated for both the 16 channel visual coil and the 32 channel whole head coil for one dimensional accelerations, both left-right and anterior-posterior, using data from coil sensitivity profiles and noise correlations as acquired from subject measurements. g-factors for the Visual Arc coil were lower than those for the 32 channel whole brain coil for all acceleration factors, as seen in Fig 2.15. At $R=4$, the maximum g-factor for the for the 32 channel coil was 1.5 times that of the Visual Arc coil and almost 3 times as much at $\mathrm{R}=6$, as plotted in Fig 2.16. Fig 2.17 shows the SENSE reconstructed images for both coils.

Functional MRI data was acquired at $1.2 \mathrm{~mm}$ (Fig 2.18) and $0.8 \mathrm{~mm}$ (Fig 2.19) isotropic resolution using both coils, as shown in the activity maps. The maps are thresholded to the same $t$-value of $3.5(\mathrm{p}<0.01)$ across both coils and both resolutions. Through a combination of a wide visual angle and densely packed receiver coils in the region of interest, the Visual Arc coil shows activation deeper into the occipital lobe, both in the superior-inferior and anterior-posterior directions at 
Chapter 2. A specialized multi-transmit head coil for high resolution fMRI of the human visual cortex at 7T

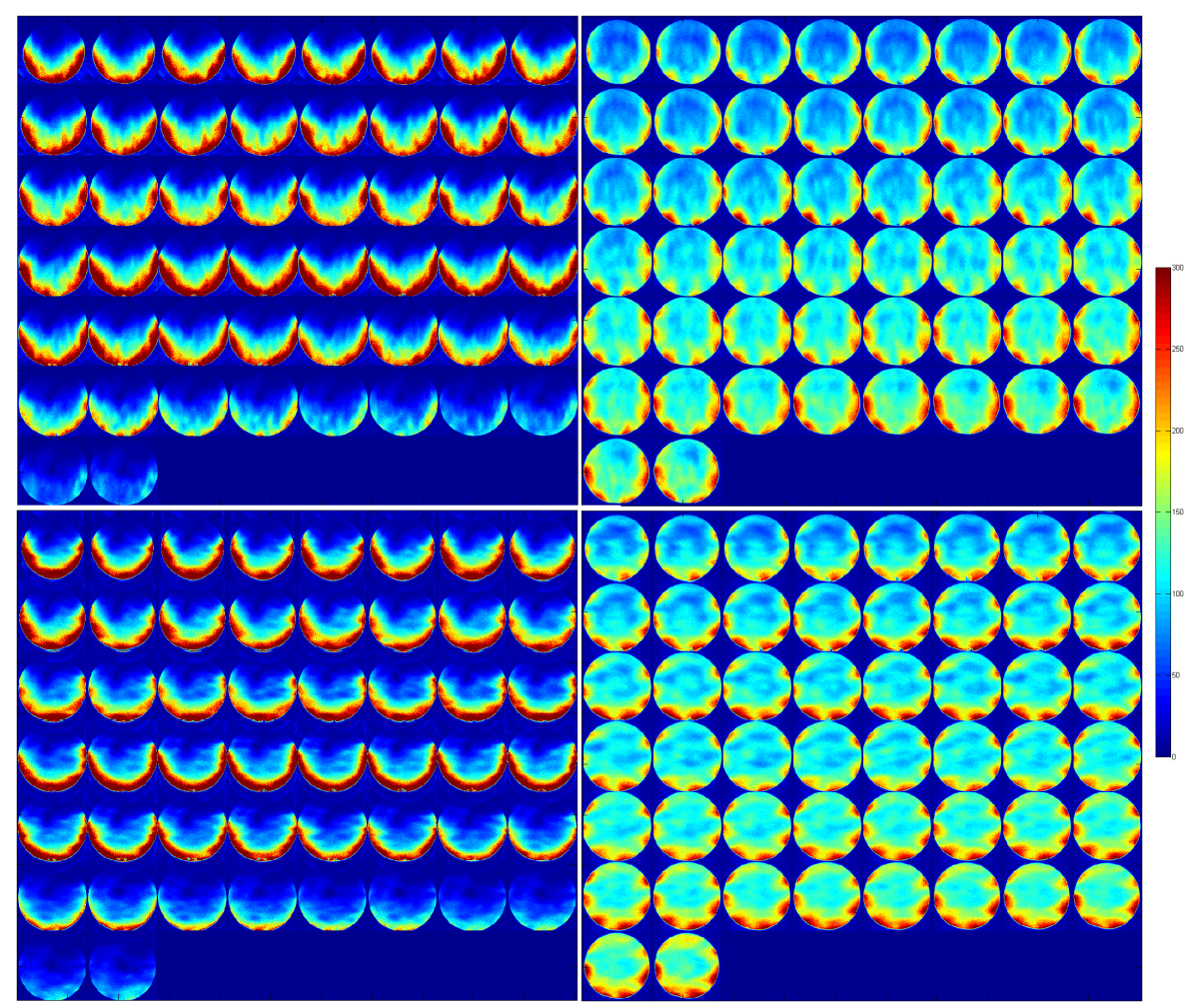

Figure 2.12: tSNR maps at $1.2 \mathrm{~mm}$ isotropic, for an transverse slice stack using a spherical phantom for the (left) Visual Arc coil and (right) the 32 channel whole head coil using L-R phase encoding (top) and A-P phase encoding (bottom). 


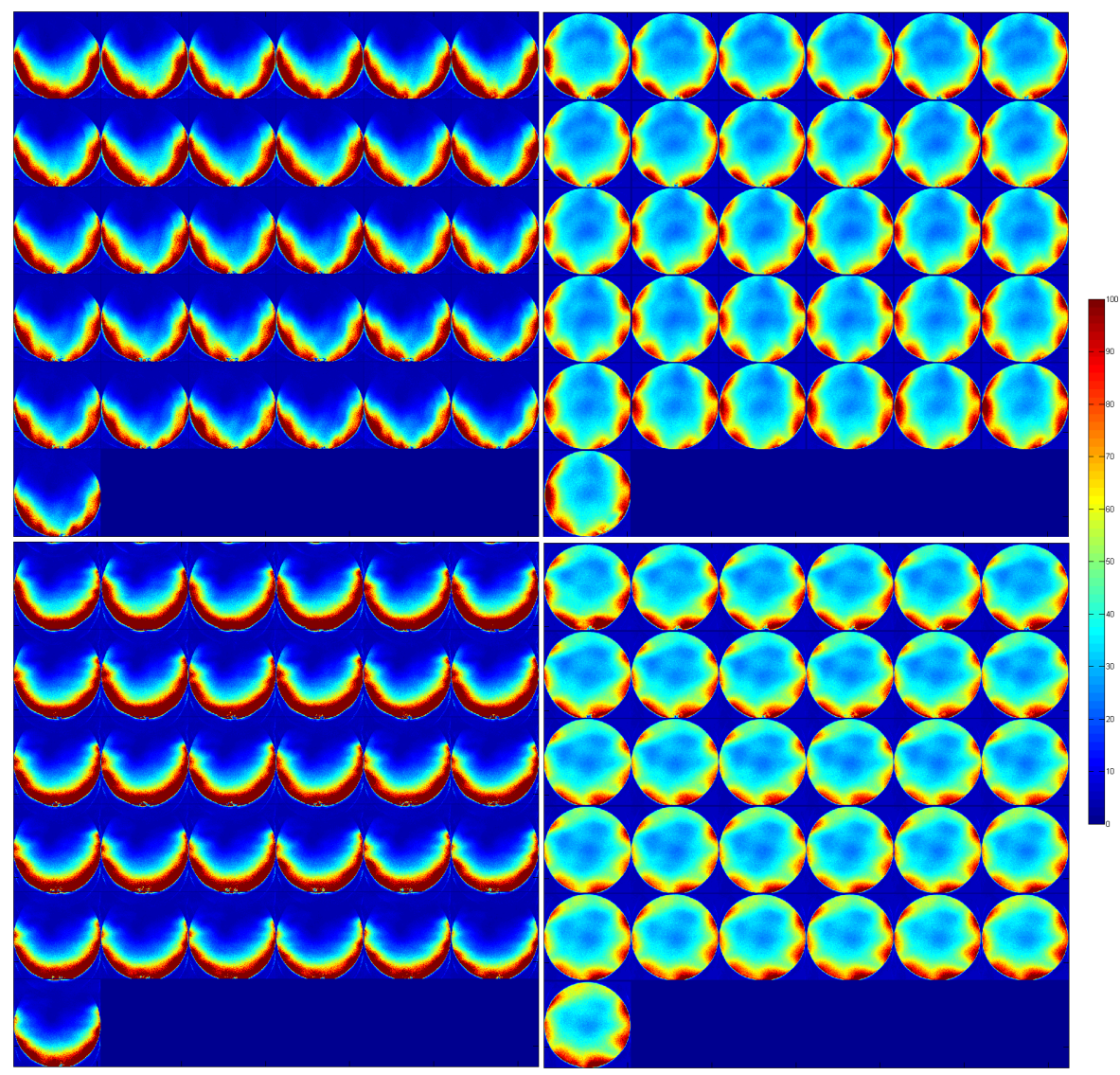

Figure 2.13: tSNR maps at $0.8 \mathrm{~mm}$ isotropic, for an transverse slice stack using a spherical phantom for the (left) Visual Arc coil and (right) the 32 channel whole head coil using L-R phase encoding (top) and A-P phase encoding (bottom). 
Chapter 2. A specialized multi-transmit head coil for high resolution fMRI of the human visual cortex at $7 T$

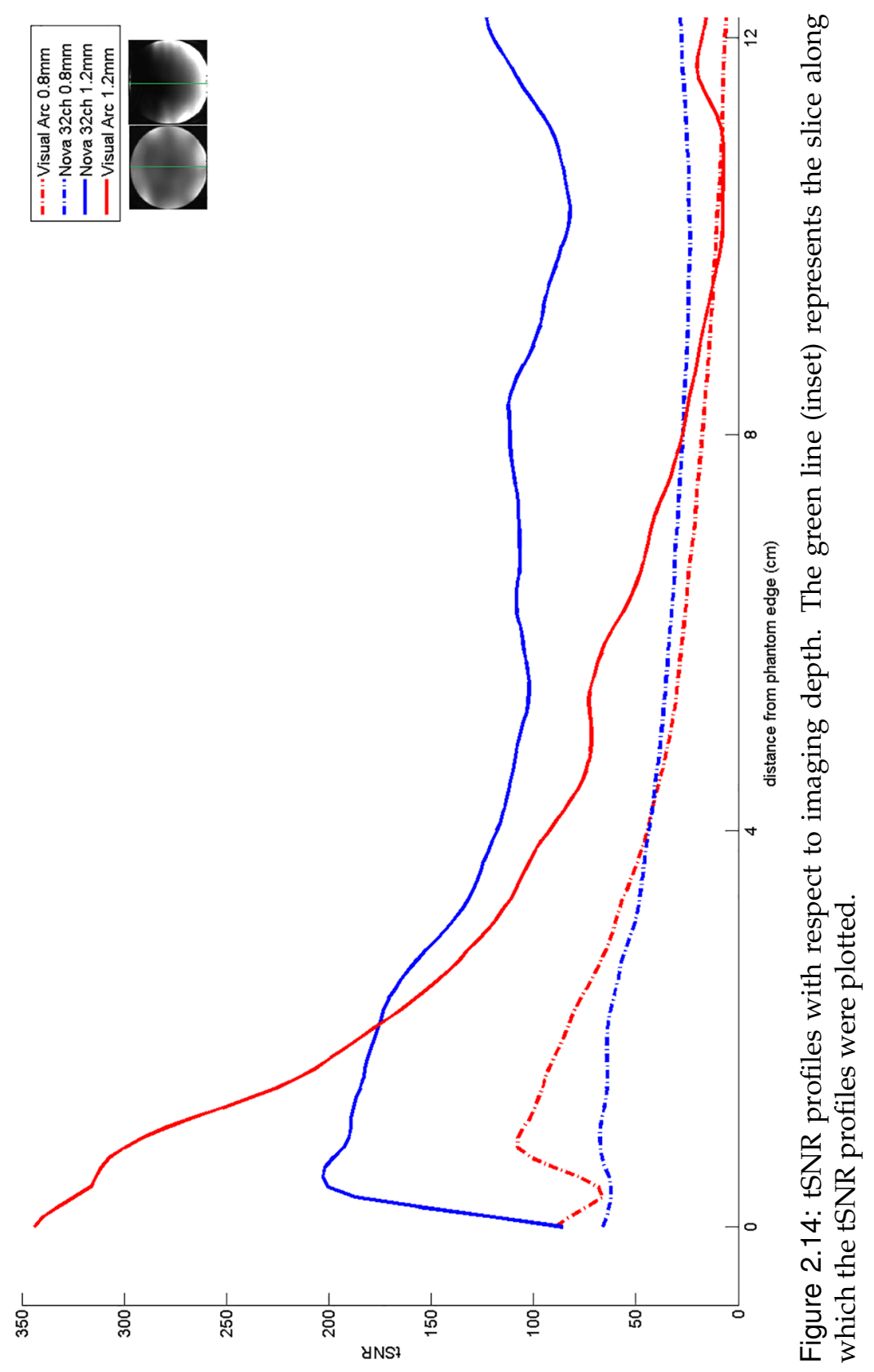




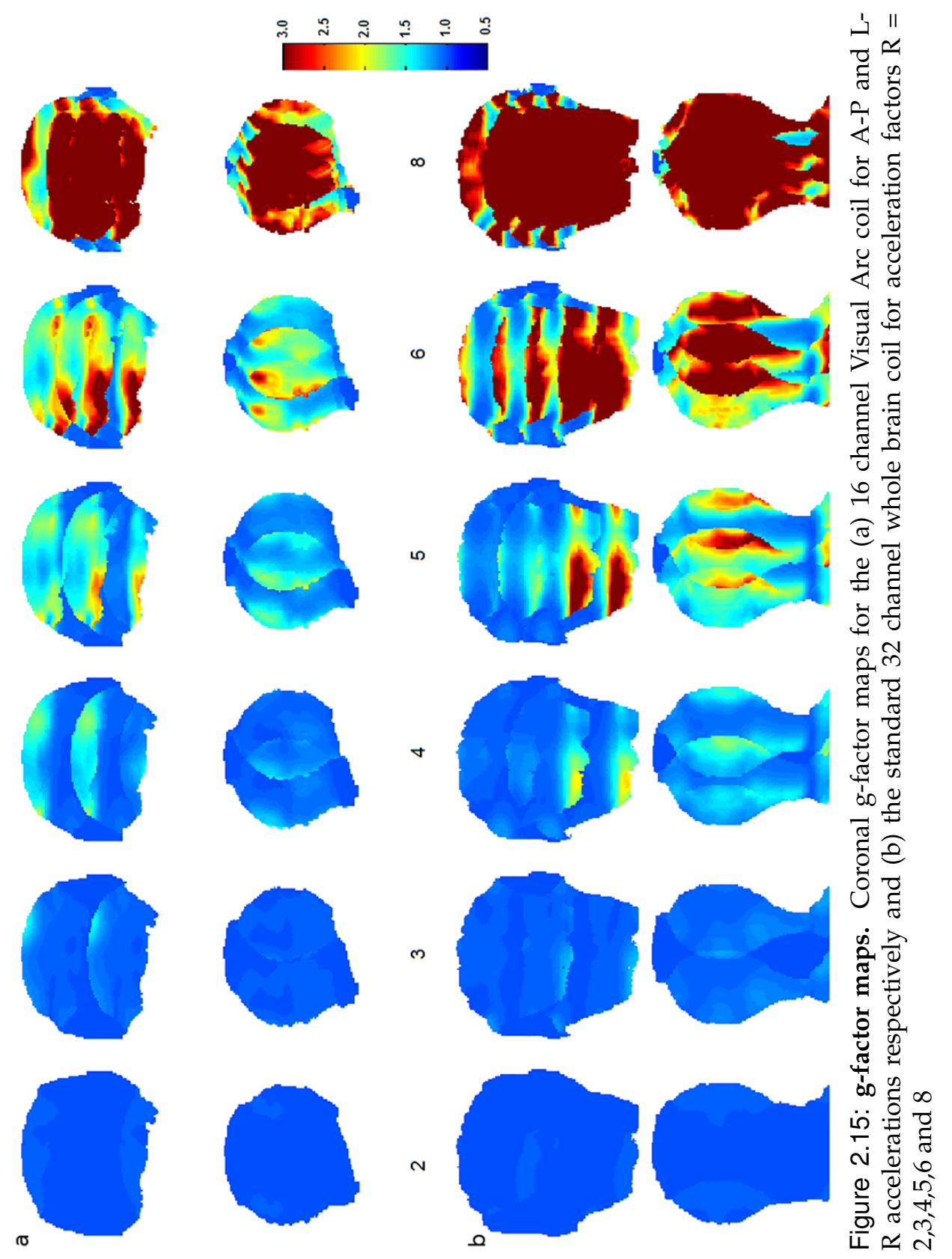


Chapter 2. A specialized multi-transmit head coil for high resolution fMRI of the human visual cortex at $7 T$

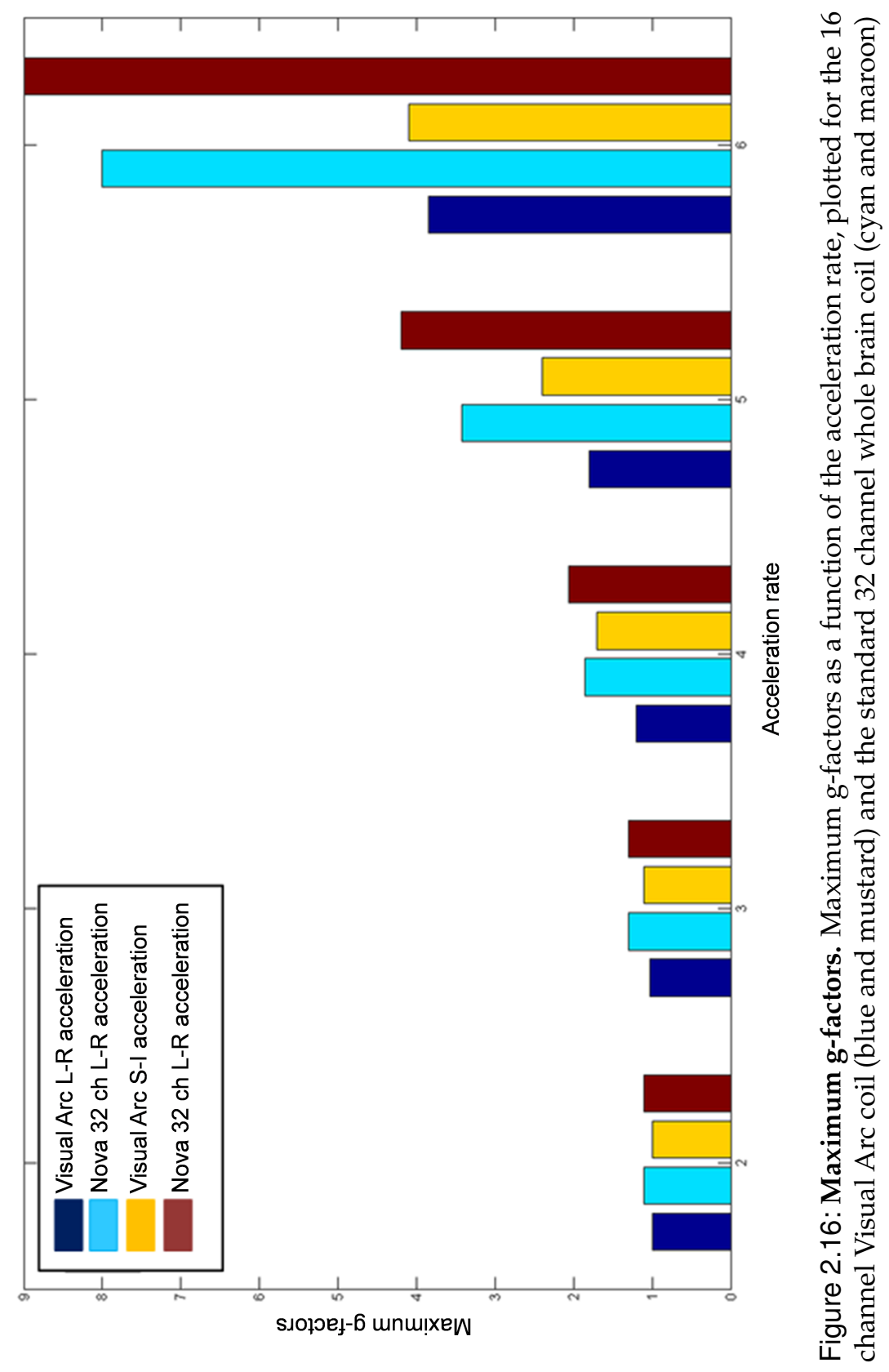



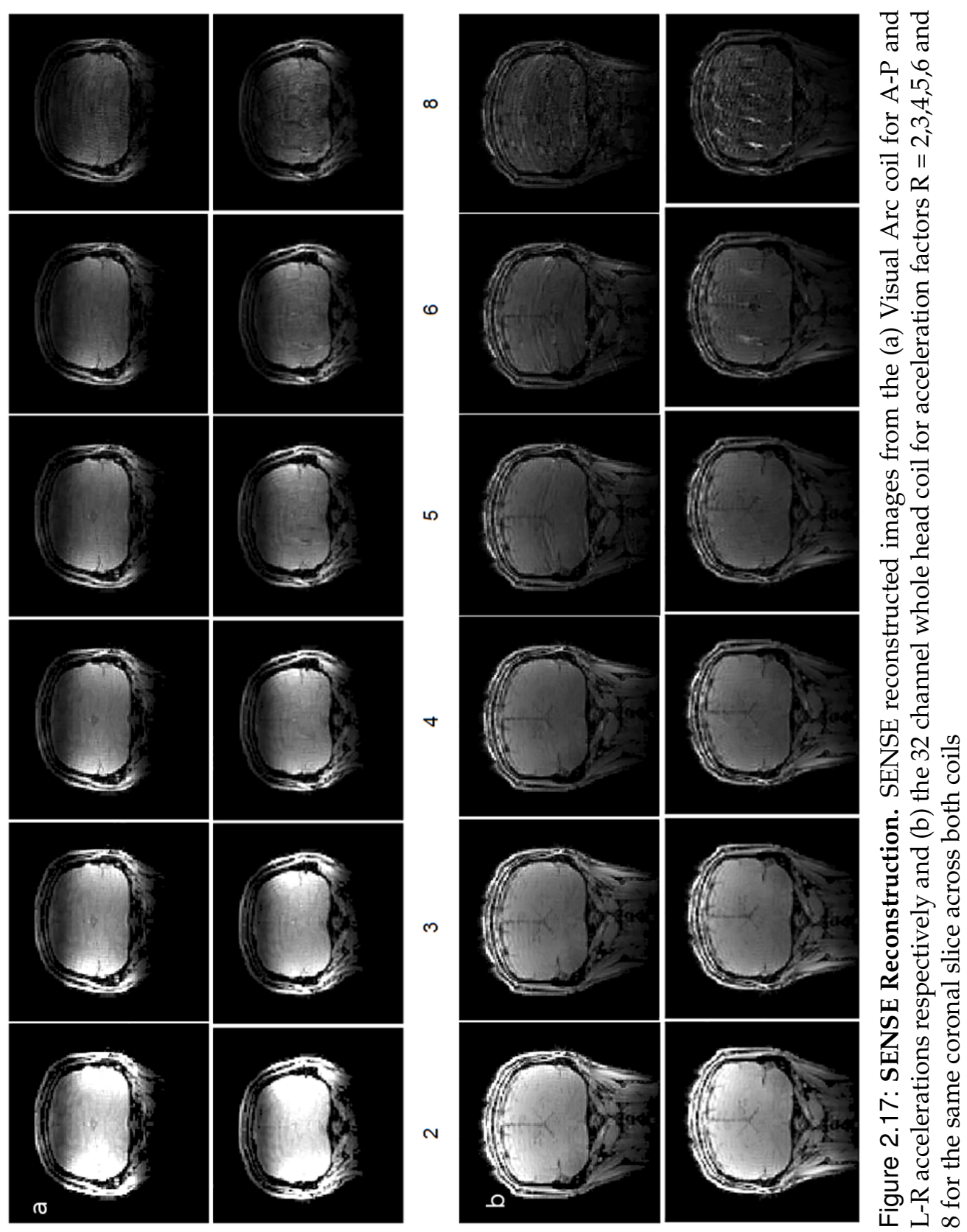
Chapter 2. A specialized multi-transmit head coil for high resolution fMRI of the human visual cortex at 7T

$1.2 \mathrm{~mm}$ resolution. The resulting activation signals at $0.8 \mathrm{~mm}$ isotropic resolution conform to a similar pattern, indicating that notwithstanding the small voxel size, fMRI responses can be measured adequately if appropriate imaging hardware are used.

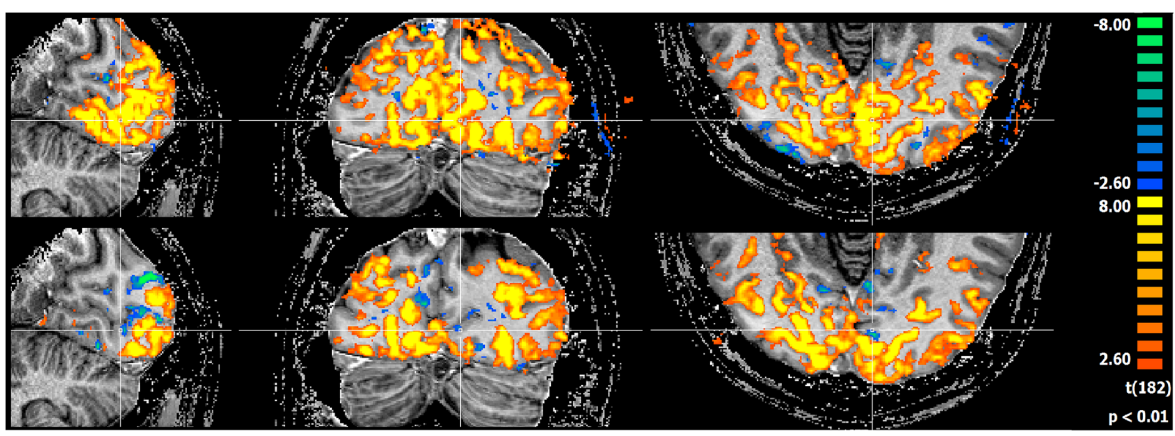

Figure 2.18: fMRI at $\mathbf{1 . 2} \mathbf{~ m m}$ isotropic. fMRI activation maps along 3 planes for the 16 channel visual coil (top) and the 32 channel head coil (bottom) at $1.2 \mathrm{~mm}$ isotropic

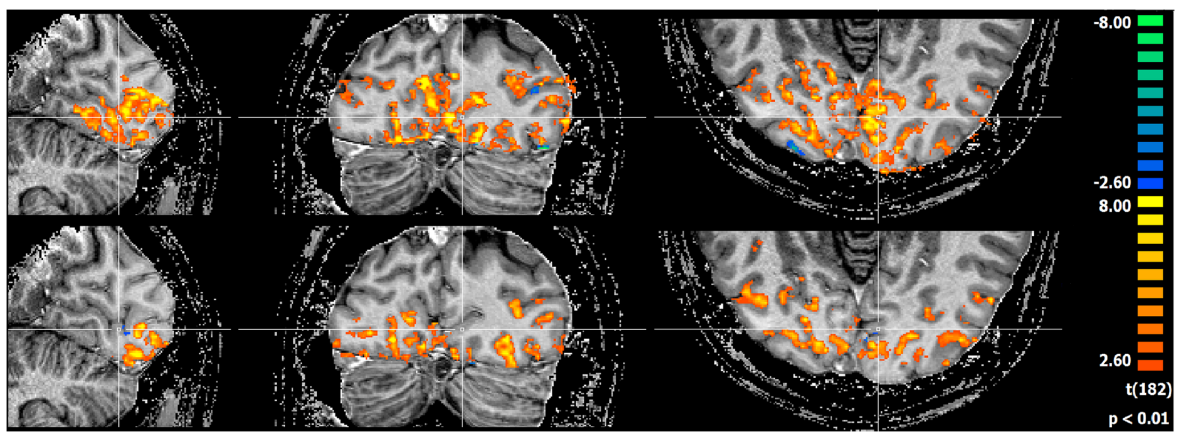

Figure 2.19: fMRI at $\mathbf{0 . 8} \mathbf{~ m m}$ isotropic. $\mathrm{fMRI}$ activation maps along 3 planes for the 16 channel visual coil (top) and the 32 channel head coil (bottom) at $0.8 \mathrm{~mm}$ isotropic 


\subsection{Discussion}

In this study, we constructed and characterized a semi-cylindrical phased-array transmit coil and a shape-optimized, 16 channel receive coil targeted at acquiring high-resolution anatomical and functional MRI data at 7T. The coil's performance was evaluated through bench tests, phantom and in-vivo imaging. The close fitting transmit coil does not require otherwise time-consuming tune and match adjustments for different subjects, lowering experimental setup time. The placement of the 2 rows of receive arrays not only covers the entire visual cortex, but also lies conformal to it, making sure that the coils are as close as possible to the region of interest and enhancing their receive sensitivity when compared to that of the 32 channel whole head coil. We did observe a slight frequency shift between loaded and unloaded cases, which was compensated for by using the human-torso shaped phantom during bench tests. Differences were also observed between the simulated and measured $B_{1}^{+}$transmit maps, which can be attributed to the shape and positional placement differences between the in-vivo subject and the voxel model used for simulation.

It is also noted that the $B_{1}$ transmit and receive profiles show the expected asymmetry at high fields while maintaining a homogeneous excitation in our targeted ROI (namely the primary and secondary visual areas), as is the case here. One way of countering this issue is by shifting the transmit and receive coils or arrays in opposing directions, by the same angle, along a circular or curved former [85, 66]. Although this would have been possible to achieve with the transmit coils on the Visual Arc, the corresponding shift of the receive array would only be possible with an unsymmetrical holder layout [Figure 2.1(C)], leading to more restricted visual openness. We did not pursue this option since we aimed to maintain full presentation capabilities but would consider it for future work.

The mean noise correlation between receive coil elements was a respectable $19 \%$; however, the worst-case correlations of $54 \%$ were found 
in adjacent coil elements in the same column. This high correlation is due to (1) insufficient overlap and thus decoupling between loops that traverse the curved surface of the former and (2) due to shared resistance acquired through the sample.

The image SNR measured shows a 1.35 fold increase in mean SNR and a 1.44 fold increase in maximum SNR for the Visual Arc coil over the standard head coil (in the occipital lobe), and this can be attributed to a combination of a tight-fitting former and smaller coil loops. This is further evident in the tSNR maps, whereby we are able to achieve sub-millimeter resolutions, allowing us to image the visual cortex into sulcal depths. The g-factors for the Visual Arc were substantially lower than those for the 32 channel whole head coil, especially at acceleration factors intrinsically associated with fMRI data acquisition $(\mathrm{R}=3,4)$ though the layout of the receive coils only allowed us to accelerate in the left-right direction.

Previous studies involving smaller FoV's covered by extremely high density 16 channel phased-array layouts of $1 \times 2 \mathrm{~cm}^{2}$ small coils at 7T have been reported by Petridou et al [71]. While we did not include such a small FoV array in our comparison, such arrays are expected to and do have very high SNR for cortical structures in the immediate vicinity of the coils. We however pursued a path of using larger coils capable of a more extensive FoV because of the limited coverage and the inherent rapid fall-off of extremely small loops. Nevertheless, we expect that in the future high density arrays covering perhaps even larger cortical regions with channel counts beyond 32 will play a significant role in high resolution fMRI.

For fMRI tests, the combination of a wide-angle subject field-of view (due to the frontal-open design of the Visual Arc) and conformal, small receive coils allow for efficient visual task presentation and increased sensitivity to BOLD response, allowing for sub-millimeter fMRI acquisition. The added activation on the anterior side is due to the open design of the coil, as this cortical region represents more eccentric retino- 
topic positions. We even notice negative activation with the 32 channel whole head coil in the same regions. They reflect a well known phenomenon of lateral inhibition which causes negative BOLD adjacent to stimulated areas [86].

The benefits of the 4 channel transmit array could be further enhanced by incorporating parallel transmit hardware that would allow for better control over $B_{1}^{+}$fields and possibly help reduce SAR effects even further. At the receive end, it is abundantly clear that denser coil arrays would be beneficial for human brain imaging, ideally using coils of an optimised diameter (likely around $4 \mathrm{~cm}$ ) at 7T. Future work would involve increasing the number of receive channels beyond 32 and towards high channel counts ( 96 and even 128, as already presented by Wiggins et al [50] at 3T) while increasing the overall volumetric coverage to be able to image the entire human brain. 



\section{High resolution anatomical and quantitative MRI of the entire human occipital lobe ex vivo at $9.4 \mathrm{~T}$}

Contents

3.1 Introduction $\ldots \ldots \ldots \ldots$

3.2 Methods . . . . . . . . . . . . . . . . . . 71

3.3 Results . . . . . . . . . . . . . . . . . . . . . . . 79

3.4 Discussion . . . . . . . . . . . . 88

Adapted from: Sengupta S., Fritz F. J., Harms R. L., Hildebrand S., Tse D. H. Y., Poser B. A., Goebel R., and Roebroeck A. High resolution anatomical and quantitative MRI of the entire human occipital lobe ex vivo at 9.4T. NeuroImage, 168:162-171, 2017 


\section{Abstract}

Several magnetic resonance imaging (MRI) contrasts are sensitive to myelin content in gray matter in vivo which has ignited ambitions of MRI-based in vivo cortical histology. In addition, quantitative MRI has been shown to have clear advantages for cortical MRI based histology. Ultra-high field (UHF) MRI, at fields of 7T and beyond, is crucial to provide the resolution and contrast needed to sample contrasts over the depth of the cortex and get closer to layer resolved imaging. Exvivo MRI of human post mortem samples is an important stepping stone to investigate MRI contrast in the cortex, validate it against histology techniques applied in situ to the same tissue, and investigate the resolutions needed to translate ex vivo findings to in vivo UHF MRI. Here, we investigate key technology to extend such UHF studies to large human brain samples while maintaining high resolution, which allows investigation of the layered architecture of several cortical areas over their entire 3D extent and their complete borders where architecture changes. A 16 channel cylindrical phased array receive coil was constructed to image a large post mortem occipital lobe sample $\left(\sim 80 \times 80 \times 80 \mathrm{~mm}^{3}\right)$ in a wide-bore $9.4 \mathrm{~T}$ human scanner with the aim of achieving high-resolution anatomical and quantitative MR images. Compared with a commercial human head coil at 7T, the maximum Signal-to-Noise ratio (SNR) was increased by a factor of more than four. Although the transmit profile with a circularly polarized transmit mode at $9.4 \mathrm{~T}$ is very inhomogeneous over the large sample, this challenge was successfully resolved with parallel transmit using the kT-points method. Using this setup we achieved $60 \mu \mathrm{m}$ anatomical images for the entire occipital lobe showing increased spatial definition of cortical details compared to lower resolutions. In addition, we were able to achieve sufficient control over SNR, $B_{0}$ and $B_{1}$ homogeneity and multi-contrast sampling to perform quantitative $T_{2}{ }^{*}$ mapping over the same volume at $200 \mu \mathrm{m}$. Markov Chain Monte Carlo sampling provided max posterior estimates of quantitative $T_{2}{ }^{*}$ and their uncertainty, allowing delineation of the stria of Gennari over the entire length and width of the calcarine sulcus. We discuss how custom 
parallel transmit and receive radiofrequency coils built to specific large post mortem sample sizes can provide a future platform for UHF cortical layer-specific quantitative MRI over large fields of view. 



\subsection{Introduction}

With increases in main field strengths, from 3T to 7T and beyond, and increases in the number of channels in phased-array RF coils, it has become increasingly clear that RF coils specifically built to subject or sample size and shape can offer very large advantages [75, 48, 88, 68]. Although this has specially been true for human in-vivo 7T coil designs, this approach is less developed in the field of ex-vivo tissue MR. Investigations of human ex-vivo brain samples in particular have therefore been limited to either tissue samples that fit pre-clinical MR setups, or the contrast and Signal-to-Noise ratio that can be achieved with a non-specialized setup, such as an in-vivo head coil.

Recently a number of investigations have shown that several magnetic resonance imaging (MRI) contrasts (such as $T_{1}, T_{2}, T_{2}^{*}$ and phase) show sensitivity to iron and, especially, myelin content in gray matter in vivo [89, 90, 91, 92, 93, 94, 95, 96] which has ignited ambitions of MRI-based in vivo histology [60, 92, 97, 93, 98]. In some of these investigations quantitative MRI, providing quantitative contrasts in physical units rather than $T_{1}$ or $T_{2}{ }^{*}$ weighted acquisitions, has been shown to have clear advantages for cortical MRI based histology [92, 97, 99]. However, so far in vivo resolution, even at UHF, has not been sufficient to resolve individual cortical layers. This is why many studies use ex vivo MRI of human post mortem samples as a stepping stone to investigate MRI contrast in the cortex, validate it against histology techniques applied in situ to the same tissue, and investigate the resolutions needed to translate ex vivo findings to in vivo UHF MRI [60, 100, 101, 102, 103, 104, 105].

Ex vivo imaging involves scanning post-mortem tissue over long scan sessions (several hours to a couple of days) at UHF which can yield ultra-high resolution datasets far below the millimetre scale while maintaining a sufficiently high Signal-to-Noise ratio (SNR) for the acquired images. Ex vivo UHF MRI to examine human brain tissue samples has been predominantly performed on preclinical or animal MRI 
systems [106, 107, 108, 109, 110, 111, 112, 113]. This takes advantage of the high field strength and increased gradient performance of preclinical systems but is often limited to small human tissue samples (smaller than about $20 \times 20 \times 20 \mathrm{~mm}^{3}$ ), restricting size of the brain region examined. Alternatively, relatively small tissue samples have been investigated on a human 7T MRI system either using a commercial head RF coil or a small custom built RF coil (e.g. [101, 102, 103, 114]. Extending such UHF studies to large human brain samples, while maintaining high resolution, would be an important step because it would allow investigations of the architecture of several cortical areas over their entire extent and their complete borders where architecture changes, which is very relevant to the translation to the in vivo situation. This requires sample-size specific RF-coils used in a large bore system [115, 101, 102] which would be difficult in a preclinical system due to space constraints.

Therefore, in this study, we focus on a custom built UHF RF-coil as a key technology for high resolution ex vivo anatomical and quantitative MRI. We designed and characterised a cylindrical phased-array receive $(\mathrm{Rx})$ coil for use in a large-bore $9.4 \mathrm{~T}$ system and compared its performance with that of a standard whole head array coil at 9.4T. The two specific aims in enabling cortical laminar discrimination over large FoVs are the following. First, we aim to achieve high resolution anatomical images $(<100 \mu \mathrm{m})$ for a large $\left(\sim 80 \times 80 \times 80 \mathrm{~mm}^{3}\right)$ human occipital lobe sample. Second, we aim to achieve sufficient control over SNR, $B_{0}$ and $B_{1}$ homogeneity and multi-contrast sampling to perform quantitative $T_{2}{ }^{*}$ imaging over the same volume at $200 \mu \mathrm{m}$. We use the primary visual cortex along the entire calcarine sulcus as a showcase to illustrate the capacity to delineate architectural details over a large surface of cortex. We also highlight how the interaction of RF coil hardware, $B_{1}$ homogenisation and data analysis must be tuned to achieve optimal results. 


\subsection{Methods}

\section{Mechanical RF-coil and sample container construction}

The coil was constructed on a hollow cylindrical transparent polycarbonate (PC) former and consists of 16 receive coils built in a phasedarray layout on the outer surface of the hollow cylinder (Fig 3.1A). The receive array former has an external diameter of $90 \mathrm{~mm}$ and a wall thickness of $3 \mathrm{~mm}$, allowing an inner diameter of $84 \mathrm{~mm}$ to accommodate ex vivo samples. The receive coils were constructed using $1 \mathrm{~mm}$ thick copper wires coated with a thin layer of polyimide for insulation - with each individual coil being $42 \mathrm{~mm}$ in diameter - and laid out in a $8 \times 2$ matrix of 16 coils that provide a cylindrical region of interest for imaging purposes. A critical overlap (of 0.20 to 0.25 times the loop diameter of each coil) was maintained between all coils to minimize mutual inductance between coils and to ensure mutual decoupling. A layer of Kapton tape was inserted over each coil such that the overlapping areas of each coil pair would be sufficiently insulated from each other. Two distributed capacitors for each circular loop were divided symmetrically along the coil loop. A detailed schematic of the individual receive coil element is shown in Fig 3.1D. For the sample housing, a cylindrical container was designed in SolidWorks and 3D printed using SOMOS XC1112 (DSM, Heerlen, NL) material (Fig 3.1C). This particular material was chosen as it possesses susceptibility close to that of water, helping us reduce susceptibility-induced artifacts. The container was designed to have an outer diameter of $82 \mathrm{~mm}$ and a wall thickness of $1 \mathrm{~mm}$, allowing for an internal diameter of $80 \mathrm{~mm}$. With a length of $90 \mathrm{~mm}$, the sample container was able to accommodate large post-mortem brain samples such as the single human occipital lobe that was used here. The container was closed using a $5 \mathrm{~mm}$ thick 3D printed cover with a sealing groove along its circumference. As sealant either silicon pressure paste (Dow Corning, Seneffe, BE) or standard 
water-proof quick drying silicone sealant was applied in the groove of the container cover which was then pressed down onto the container to provide a water-tight fit. A hole was drilled onto the container cap in order to fashion a degassing port, which was then plugged using a nylon screw and a nitrile O-ring. Additional Kapton tape was applied to the outside of the container to secure the cover and provide a tight fit in the coil former.

\section{RF-coil circuitry}

Each receive coil was connected to a lattice (LC) balun circuit [77, 48] and a tuning and matching network consisting of high-voltage ceramic trimmer capacitors $C_{T}$ and $C_{M}$ (1-10pf, Johanson Technology, CA, USA) for tuning the coil to resonance at 9.4 Tesla and matching

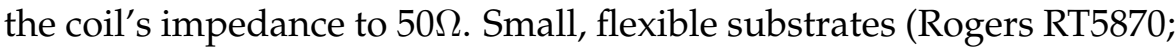
Rogers Corporation, Gent, BE) were used to construct passive detuning circuitry consisting of an LC circuit in parallel with a high voltage diode was placed on the same board as the balun circuit. All coils were tuned and matched to $400 \mathrm{MHz}$ and connected to low inputimpedance preamplifiers (WMA9RA, WanTcom Inc., MN, USA) using $\lambda / 4(130 \mathrm{~mm}$ ) length coaxial cable (Huber-Suhner, K02252D), to ensure preamplifer decoupling between individual coil elements by transforming the high impedance at the coil output to the corresponding low impedance at the preamplifier input [21, 77]. The balun circuit incorporates an active detuning circuit across the matching capacitor, using the balun inductor and a PIN diode. With the PIN diode forward biased, the parallel resonant LC circuit adds a high impedance in series with the coil loop, effectively blocking current flow in the receive loop during transmit. The cable length was adjusted for each coil to achieve preamplifier decoupling without requiring further lumped element phase shifting circuitry. The preamplifiers were arranged in a circular layout mirroring the receive coil layout and are placed on a circular "preamplifier motherboard" containing the required circuitry 
for providing the PIN diode voltages for active detuning of the receive elements during transmit. The preamplifiers were also arranged along the $B_{0}$ field or the z-direction of the magnet to minimize any Hall effect which might affect the field effect transistors (FETs) used in these preamplifiers [116]. Cable traps on the output of each preamplifier were required and implemented - by fashioning a solenoid (2 turns, 4 $\mathrm{mm}$ diameter) from the coaxial cable connecting the preamplifier output to the plug that connects to the magnet bed, soldering a variable capacitor across its ends and tuning it to the resonant frequency of 400 $\mathrm{MHz}$, while being positioned outside the immediate RF receive loop locations. The traps significantly reduced common-mode currents and were found to be essential to suppress interactions with the transmit coil.

For transmit, a separate 16 channel pTX transmit coil [65] was used. The receive coil assembly was designed to be concentric to the transmit coil in order to allow for easy integration between transmit and receive sections (Fig 3.1B).

\section{Sample preparation}

Tissue used for imaging was obtained post mortem from the left hemisphere of a male subject, without known neurological or psychiatric disorders. The tissue donor gave his informed and written consent to the donation of his body for teaching and research purposes regulated by the Dutch law for the use of cadavers for scientific research and education. Accordingly, a handwritten and signed codicil from the donor, posed when still alive and well, is kept at the Department of Anatomy and Embryology, Faculty of Health, Medicine and Life Sciences, Maastricht University, Maastricht, The Netherlands. The approximately 80 $x 80 \times 80 \mathrm{~mm}^{3}$ formalin-fixed occipital lobe of the left hemisphere was first immersed in 6 times its volume of phosphate-buffered solution (PBS) for 6 weeks to wash out formalin and rehydrate the sample. Af- 
Chapter 3. High resolution anatomical and quantitative MRI of the entire human occipital lobe ex vivo at $9.4 T$
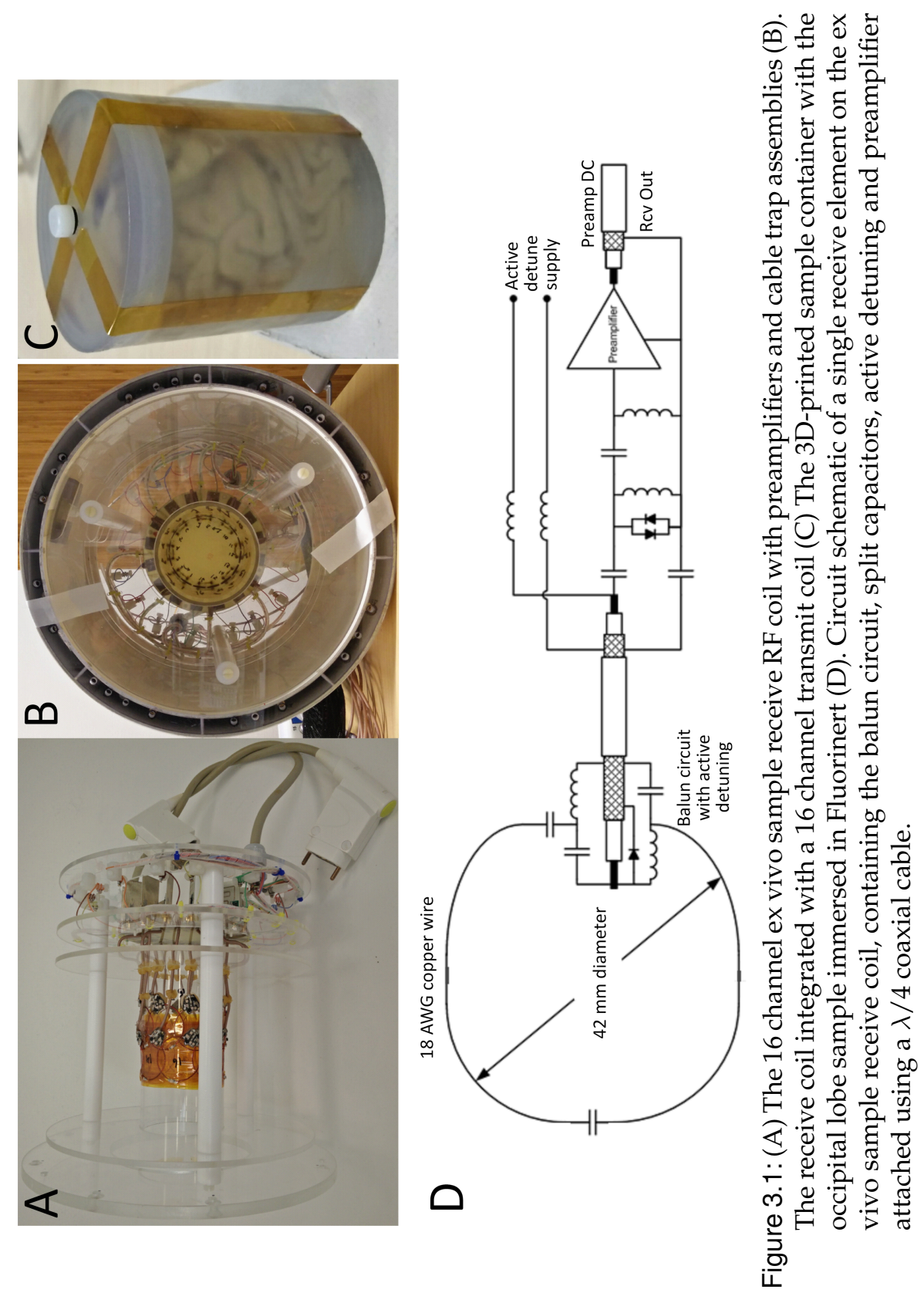
ter washing, the sample was placed in the 3D printed cylindrical container and embedded in proton-free, susceptibility matched fluid Fluorinert FC-3283 (3M, Delft, NL) for imaging. The container was then sealed and the cover pressed down to allow the silicone to distribute evenly along the lid. Using a syringe, Flourinert was injected into the cylinder to replace air bubbles and the degassing port sealed.

\section{RF-coil bench measurements}

Bench tests were performed using an Agilent HP E5071C ENA Series network analyser, a customized coil-plug bed and a cylindrical phantom ( $49.8 \%$ demineralized water, $48.8 \%$ sucrose, $1.3 \% \mathrm{KCl}, 0.10 \%$ Dowicil; Max Planck Institute for Biological Cybernetics, Tübingen, Germany ) as load. Measurements performed checked for loaded $\left(Q_{L}\right)$ and unloaded $\left(Q_{U}\right) \mathrm{Q}$ ratios, receive coil decoupling and preamplifier decoupling for each coil element. The bench tests were undertaken to validate coil tuning and matching, preamplifier decoupling for each coil element and active coil detuning.

The coil quality factor ratio $\left(Q_{U} / Q_{L}\right)$ was measured for a single receive coil element, using a dual-loop decoupled ( $50 \mathrm{~dB}$ ) inductive probe coupled to a single Rx element: once as a single element outside the receiver array and once within the populated array while keeping all other receive elements in a detuned state. Each loop on the receive array was tuned to the Larmor frequency of $400 \mathrm{MHz}$ and matched

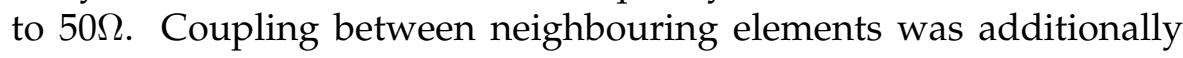
measured through $S_{21}$ measurements by connecting the coil outputs to the network analyser, keeping all other elements detuned. Using this setup, the overlap between neighbouring elements could be further optimized to ensure minimal mutual inductance. Preamplifier decoupling of a single loop was measured as the change in the $S_{21}$ measurement using a pair of decoupled pickup probes, when the coil element was first power matched to $50 \Omega$ under load but without the 
Chapter 3. High resolution anatomical and quantitative MRI of the entire human occipital lobe ex vivo at $9.4 T$

preamplifier present and when the coil was terminated using the low input impedance preamplifier, while all other coils in the receive array were detuned. The active detuning for each receive element was measured as the difference in an $S_{21}$ measurement, between when the coil is matched to $50 \Omega$ and when detuned.

\section{MRI acquisition and analysis}

Data acquisitions were performed on a $830 \mathrm{~mm}$ human size bore research 9.4T Siemens MAGNETOM MR system (Siemens Healthcare, Erlangen, Germany) interfaced with a 16Ch parallel transmit (pTx) coil. Prior to acquiring high resolution anatomical data, $B_{0}$ and $B_{1}+$ shimming were performed. First, a localizer was acquired for spatiallocalization reference; then a fieldmap [117] and DREAM [118] protocol were acquired to characterise $B_{0}$ and $B_{1}+$ map profiles, respectively, along the sample. MATLAB (MathWorks, MA, USA) routines were used to optimise $B_{0}$ and $B_{1}+$ shimming using those acquisitions [119, 120]. Fieldmap sequences were acquired and shimmed iteratively, two to three times, to improve $B_{0}$ homogeneity.

All acquisitions were performed with a custom 3D gradient echo (GRE) pulse sequence, modified to allow large matrix sizes and to use a composite parallel excitation pulse using the kT-points technique for $B_{1}+$ homogenization [121]. An Actual Flip-angle Imaging (AFI) sequence with $\mathrm{n}=5$ [80] was acquired later to verify $B_{1}+$ homogeneity. The 3D GRE sequence was used to acquire high resolution isotropic $T_{2}{ }^{*}$ weighted data at: $500 \mu \mathrm{m}$ (Repetition time (TR)/Echo time (TE) $=$ $36 \mathrm{~ms} / 17.68 \mathrm{~ms}$, flip angle $(\mathrm{FA})=35^{\circ}$, readout bandwidth $(\mathrm{BW})=220$ $\mathrm{Hz} / \mathrm{px}$, matrix dimensions $=160 \times 156 \times 160), 100 \mu \mathrm{m}(\mathrm{TR} / \mathrm{TE}=47 \mathrm{~ms} / 22$ $\mathrm{ms}, \mathrm{FA}=49^{\circ}, \mathrm{BW}=30 \mathrm{~Hz} / \mathrm{px}$, matrix dimensions $\left.=800 \times 800 \times 832\right)$ and $60 \mu \mathrm{m}\left(\mathrm{TR} / \mathrm{TE}=46 \mathrm{~ms} / 20.1 \mathrm{~ms}, \mathrm{FA}=49^{\circ}, \mathrm{BW}=40 \mathrm{~Hz} / \mathrm{px}\right.$, matrix dimensions $=1400 \times 1400 \times 1280$ ) isotropic resolution. A multi-echo 3D GRE protocol was used using monopolar RO gradient to acquire multi- 
contrast $T_{2}{ }^{*}$ weighted data for the purpose of quantitative $T_{2}{ }^{*}$ mapping at $200 \mu \mathrm{m}$ isotropic resolution (TR/TEs $=38.5 \mathrm{~ms} / 6.45,14.69,22.93$ and $31.17 \mathrm{~ms}, \mathrm{BW}=220 \mathrm{~Hz} / \mathrm{px}$, matrix dimensions $=400 \times 400 \times 416$ ) at different flip angles $\left(25^{\circ}, 35^{\circ}\right.$ and $\left.45^{\circ}\right)$. The in-plane field of view (FoV) for all the experiments was $80 \times 80 \mathrm{~mm}^{2}$. For all the acquisitions, a 0 volt transmit power noise reference scan was acquired with matching protocol parameters (e.g. bandwidth, FoV and base resolution). Raw data was streamed off the MR system in realtime for later image reconstruction. Offline reconstruction was performed using home-made routines in python and MATLAB. No filtering was applied in the reconstruction to show the raw quality and effective resolution and signal-to-noise ratio (SNR) of the acquired data. For high resolution GRE, $T_{2}{ }^{*}$ weighted anatomical volumes Roemer reconstruction [21] was used and for quantitative $T_{2}{ }^{*}$ mapping a noise covariance corrected root of sum of squares (cov-rSoS) reconstruction [122] was used.

To compare the SNR of the ex vivo sample coil with a more widely available alternative, equivalent data was acquired in the same scanner system using a 16 channel transmit, 31 channel receive whole-head coil [65]. The excitation profiles from both measurements were acquired using the AFI sequence in pTx mode using kT-points (in the dedicated 16 channel receive coil) and AC shim (in the Head coil). A proton-density weighted GRE using monopolar readout at $1000 \mu \mathrm{m}$ isotropic resolution $\left(\mathrm{TR} / \mathrm{TE}=1000 \mathrm{~ms} / 4.0 \mathrm{~ms}, \mathrm{FA}=88^{\circ}, \mathrm{BW}=300\right.$ $\mathrm{Hz} / \mathrm{px}$, matrix dimensions $=82 \times 90 \times 80$ ) was used with a matched zero volt noise acquisition as described above. Image SNR for both coils was computed using a pseudo-replica approach [82, 83] with the subsequent noise data. We generated 100 replicas by adding random samples from the noise scan onto the GRE k-space of the standard image acquisition prior to Fast Fourier Transform (FFT), while maintaining the noise covariance between the receive channels. The final image SNR was then calculated as the ratio of the mean of the image to the standard deviation of the noise over these 100 replicas. Resulting SNR maps for the ex-vivo sample coil and for the Head coil were corrected 
by a normalized $B_{1}+$ map calculated from AFI acquisition using kTpoints. Coregistration between AFI and GRE volumes were performed before $B_{1}+$ calculation using SPM 12. This corrects any underestimation of SNR values caused by $B_{1}$ inhomogeneity leading to flip angles away from Ernst angle. Quantitative $T_{2}{ }^{*}$ mapping was performed in a voxel-based analysis approach using the GPU accelerated pythonbased Maastricht Diffusion Toolkit [123] by Markov Chain Monte Carlo (MCMC) sampling. To this end a mono-exponential decay equation including an $S_{0}$ term was defined in MDT's OpenCL-based signal modelling language. MCMC sampling was performed using the random walk Metropolis algorithm [124] with a uniform prior for $S_{0}$ and $T_{2}{ }^{*}$ with lower and upper bounds of [0 to 50] a.u. and [0.0, 100.0] $\mathrm{ms}$ respectively and an offset-Gaussian likelihood function to account for the Rician rectified noise floor. The convergence of the chains and unimodality of the sampled posterior was visually inspected for several voxels leading to the following settings: 1500 burn-in samples, 100 samples with a step of 5 . Gaussian distributions were fitted to the histograms of the final samples to obtain the mean ( $\max$ ) posterior estimate and the standard deviation of the posterior. The log of the standard deviation is reported as a measure of the uncertainty (variability, inverse precision) of the $S_{0}$ and $T_{2}{ }^{*}$ max posterior estimates. A 3D surface reconstruction was constructed of the entire left occipital lobe in BrainVoyager QX 2.8.4 (Maastricht, The Netherlands) based on the $200 \mu \mathrm{m} \mathrm{q} T_{2}^{*}$ maps. Region growing operations, followed by erosion, smoothing and then dilation steps were used to create a surface at the approximate pial surface without contamination of vessels and pia mater to visualize the macro-anatomy of the sample. 


\subsection{Results}

\section{RF coil characterization}

The unloaded $\mathrm{Q}$ factor $Q_{U}$ (indicative of coil losses) for an isolated receive element of the 16 channel ex vivo sample coil at $400 \mathrm{MHz}$, was about 248 and the loaded $\mathrm{Q}$ factor $Q_{L}$, indicative of coil and tissue losses, was 38 . For a single receive loop surrounded by 5 detuned loops in the array, the same $\mathrm{Q}$ factor measurement yielded a $Q_{U} / Q_{L}$ of $245 / 42$. All receive coils were matched between $-21 \mathrm{~dB}$ and $-28 \mathrm{~dB}$ for the occipital lobe sample. The active PIN diode detuning provided an isolation better than $28 \mathrm{~dB}$ between tuned and detuned states. The decoupling between neighboring, overlapping receive elements ranged between $-14 \mathrm{~dB}$ to $-19 \mathrm{~dB}$. Decoupling between next-nearest neighboring elements (or non-overlapping elements) of the array ranged between $-17 \mathrm{~dB}$ to $-25 \mathrm{~dB}$. Preamplifier decoupling accounted for an additional $20 \mathrm{~dB}$ of isolation. The noise correlation matrix, as shown in Fig $3.2 \mathrm{~A}$, was obtained with ex vivo scanning, with the correlation ranging between $0.2 \%$ and $51 \%$ with an average of $22 \%$.

Figure 3.2 shows the receive characteristics of the ex vivo sample coil. Fig 3.2A shows the noise correlation matrix with a maximum correlation of 0.51 and an average of 0.22 and Fig 3.2B shows the single channel images for the occipital lobe sample across the two rows of coils (clockwise across each row) with high SNR near the coil surface showing good delineation of white/gray matter (WM/GM). Fig 3.2C and 2D show coil-combined reconstructions for a $100 \mu \mathrm{m}$ isotropic GRE acquisition along a central coronal slice using a cov-rSoS reconstruction and Roemer reconstruction respectively. Substantial receive penetration to image the entire sample effectively can be seen, especially when using a coil sensitivity corrected Roemer reconstruction (Fig 3.2D).

Figure 3.3 shows a comparison between the transmit profiles at 7T with a single channel, circularly polarized (CP) transmit mode, 9.4T single 
Chapter 3. High resolution anatomical and quantitative MRI of the entire human occipital lobe ex vivo at $9.4 T$
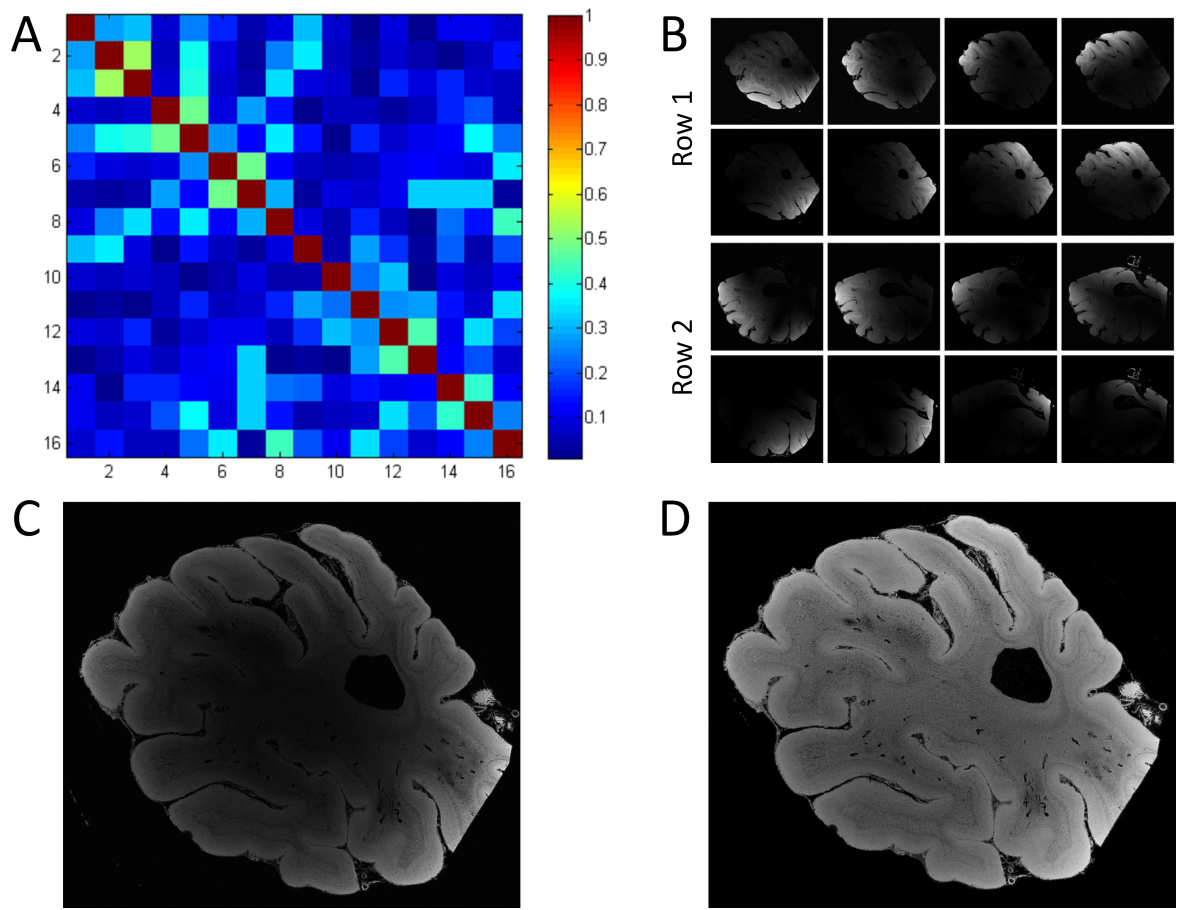

Figure 3.2: (A) Noise correlation matrix for the 16 channel receive coil (B) Individual coil sensitivity profiles for the coil, with the slices shown aligned along the center of the coil elements. $100 \mu \mathrm{m}$ isotropic GRE image using a (C) cov-rSoS reconstruction and using a (D) Roemer reconstruction respectively. 


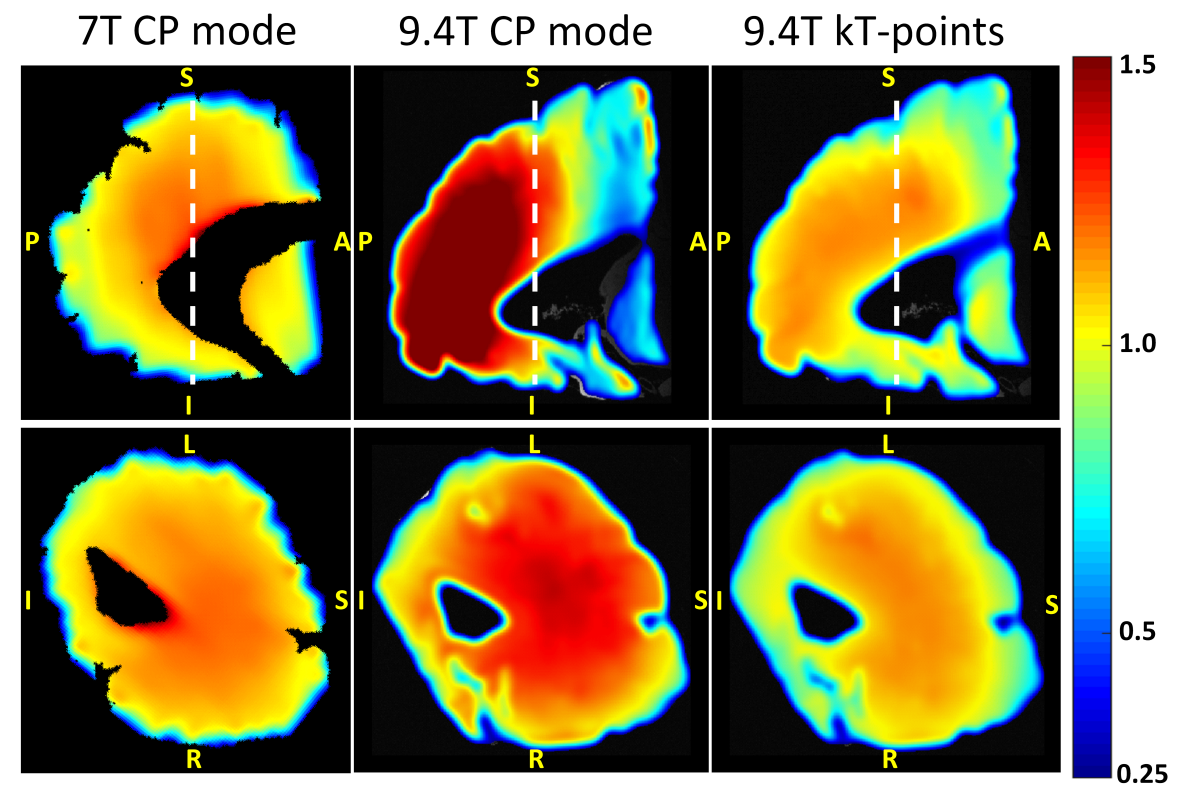

Figure 3.3: Normalized transmit $\left(B_{1}+\right)$ profile maps of 7T CP (first column), 9.4T CP (second column) and 9.4T kT-points homogenized (third column) acquisitions. Upper row shows a near sagittal slice with the dotted white line in the first row indicating the coronal slice shown in the second row.

channel CP mode and 9.4T parallel transmit (pTx) using the kT-points method. It can be seen that the 7T CP excitation profile using a birdcage transmit coil is concentrated mainly in the center of the sample and has a reasonable homogeneity, with flip angles falling off towards the periphery. The 9.4T CP excitation profile is much less homogeneous with even greater central brightness in the coronal view and a sharp falloff of flip angles from the posterior to the anterior end. However, the use of kT-points at 9.4T homogenizes the excitation profile along the sample, strongly decreasing the over-excitation in the posterior part (from $50 \%$ to almost $10 \%$ ) and increasing in the anterior part (from $-25 \%$ to $-5 \%$ in average).

Figure 3.4 shows a comparison of image SNR between the 7T CP and 
Chapter 3. High resolution anatomical and quantitative MRI of the entire human occipital lobe ex vivo at $9.4 T$

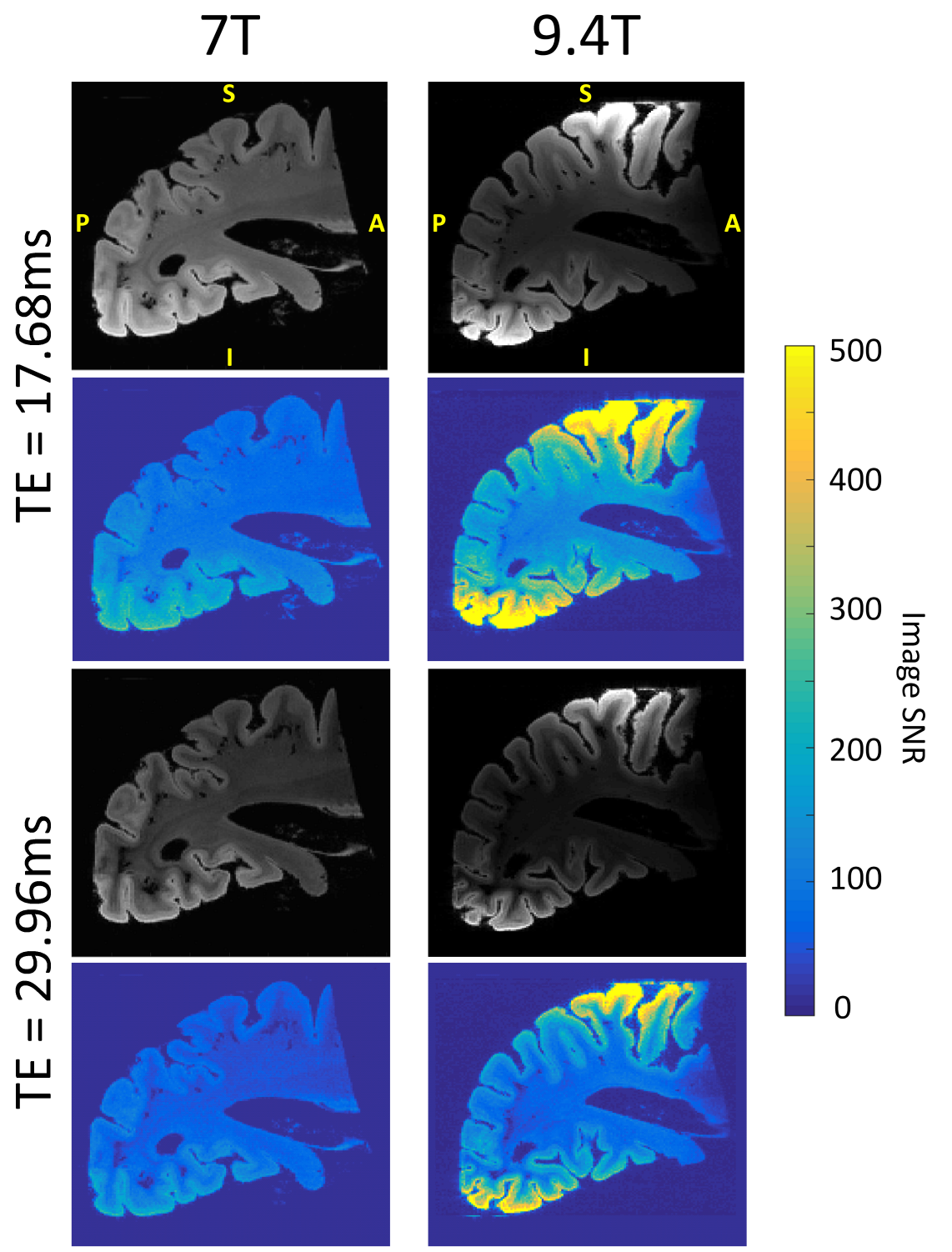

Figure 3.4: Anatomical image (gray scale) and corresponding SNR maps at 7T (left column) and at 9.4T (right column) for two GRE acquisitions at TE = $17.68 \mathrm{~ms}$ (top) and $29.96 \mathrm{~ms}$ (bottom). 
9.4T kT-points pTx setups for a $500 \mu \mathrm{m}$ isotropic GRE acquisition with matched echo times and receiver bandwidths. The echo times were chosen as relatively long TEs relevant to $T_{2}{ }^{*}$ contrast imaging. Since $T_{2}{ }^{*}$ values are shorter at $9.4 \mathrm{~T}$ than at $7 \mathrm{~T}$, in principle this choice gives an SNR advantage to the 7T acquisition. A drop-off in SNR away from the receive coils (i.e. peripheral to central) is appreciable across both acquisitions, although stronger at 9.4T. Nonetheless, the SNR at 9.4T is higher across the entire volume than in the respective acquisition at $7 \mathrm{~T}$, especially at the periphery by about a factor of 5 .

\section{Anatomical and quantitative $T_{2}{ }^{*}$ imaging}

Figure 3.5 shows successively zoomed transverse views of two $T_{2}^{*}$ weighted GRE acquisitions of the occipital lobe sample at $100 \mu \mathrm{m}$ and $60 \mu \mathrm{m}$ isotropic resolutions. No spatial filtering was used in the image reconstruction to present the quality of the raw data. The cut plane through the sample is slightly different between acquisitions as they were acquired during different sessions and oblique views which would interpolate the data were avoided for an accurate comparison. The zoom-ins on the right show the same approximate location in the sample. The anatomical detail visible (along the calcarine sulcus) across both resolutions shows the stria of Gennari (SoG) in the middle of the cortical ribbon, with a tendency of the $60 \mu \mathrm{m}$ images to show better delineation of the stria. There is also a tendency for a better definition of the pial boundary in the higher resolution $60 \mu \mathrm{m}$ dataset. The red zoom-in inset details a vessel wall which shows how a better definition of high-contrast structures can be achieved at higher resolution.

Figure 3.6 shows that quantitative $T_{2}{ }^{*}\left(\mathrm{q}_{2}{ }^{*}\right)$ mapping can be performed at $200 \mu \mathrm{m}$ isotropic resolution for the entire occipital lobe using MCMC sampling. Fig 3.6A shows sagittal views through the cov-rSoS reconstructed single echo datasets where the receive profile inhomo- 
Chapter 3. High resolution anatomical and quantitative MRI of the entire human occipital lobe ex vivo at 9.4T
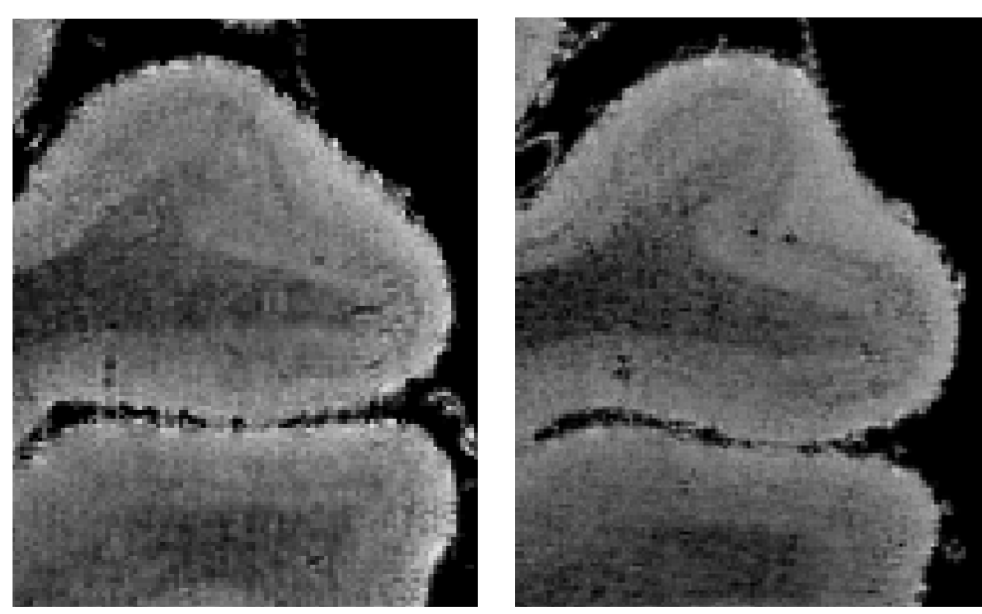

:
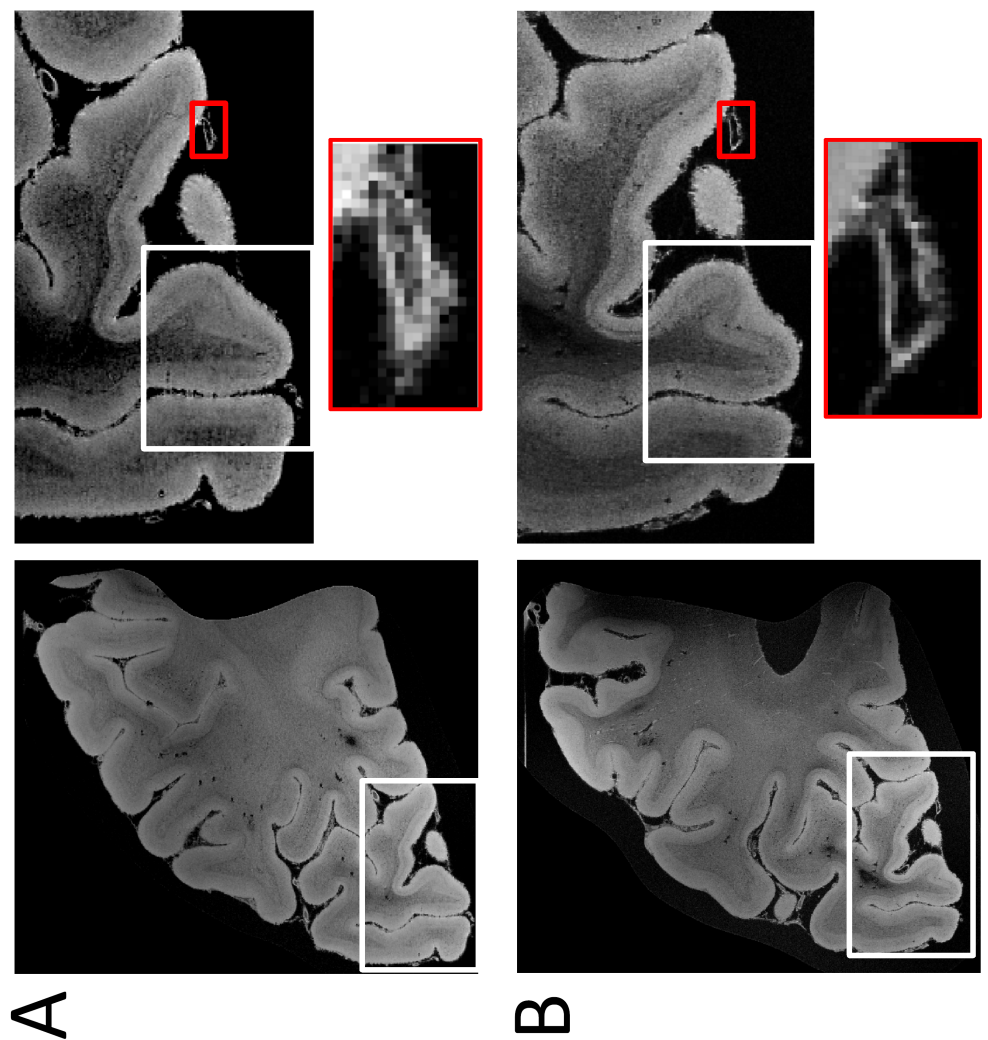

$\infty$

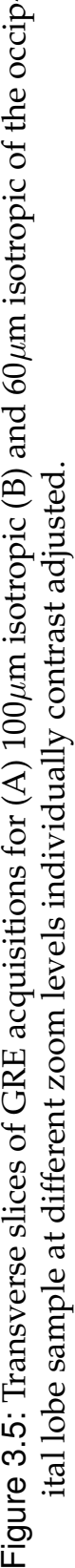




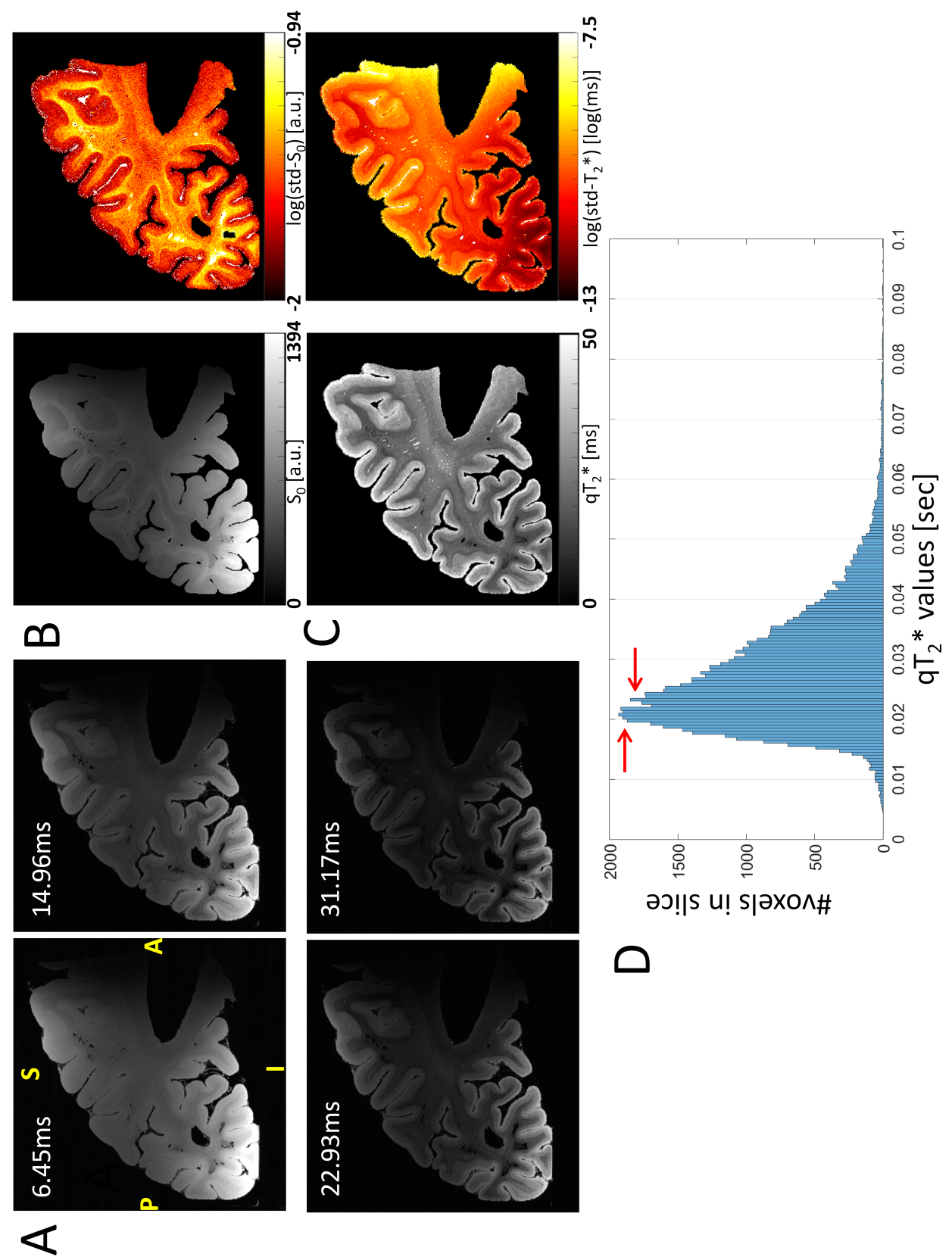

Figure 3.6: Quantitative $T_{2}{ }^{*}$ mapping of the entire occipital lobe at $200 \mu \mathrm{m}$ calculated from a multi-echo GRE acquisition using 4 TEs. (A) Individual echoes, (B) $S_{0}$ estimate, mean (left) and log standard deviation (right) and (C) $T_{2}{ }^{*}$ estimate, mean (left) and log standard deviation (right) maps. (D) A histogram of $T_{2}{ }^{*}$ along the same slice is shown where small peaks (red arrows) correspond to white and gray matter $T_{2}^{*}$ values. 
geneities are evident. Fig 3.6B and 3.6C show the estimated $S_{0}$ and $\mathrm{q} T_{2}{ }^{*}$ maps, respectively, averaged over the different FA acquisitions. The $S_{0}$ map absorbs proton density and non-quantitative transmit and receive inhomogeneities. A good $T_{2}{ }^{*}$ estimate is achieved over the whole sample with clear delineation of and gray matter and a higher $T_{2}{ }^{*}$ in the superficial gray matter layers than in the deeper layers. The $\log$ standard deviation of the posterior distribution of the MCMC chain (right images in Fig 3.6B and 3.6C) is an indication of the uncertainty of the estimates. The uncertainty of the $T_{2}{ }^{*}$ estimate is slightly higher in gray matter than in white matter and clearly lower close to the coils where signal is higher (lower left) and higher far away from the coils (on the right). The uncertainty of the $S_{0}$ estimate is higher in white matter. Fig 3.6D shows a histogram of the $T_{2}{ }^{*}$ values observed in Fig 3.6C, with small peaks corresponding to the white matter $T_{2}{ }^{*}$ of approximately $20 \mathrm{~ms}$ and a deep gray matter $T_{2}{ }^{*}$ of approximately 26 ms.

Figure 3.7A shows a 3D surface reconstruction of the entire occipital lobe using the $200 \mu \mathrm{m} \mathrm{q} T_{2}{ }^{*}$ map, showing the extent and macroanatomical features of the sample. The four coloured lines indicate the anterior-posterior (AP) locations along the calcarine sulcus (CS, indicated by arrows) for which coronal slices through the $\mathrm{qT}_{2}{ }^{*}$ map are shown in Fig 3.7B and zoomed views of the CS are shown in Fig 3.7C. The SoG is clearly visible along the cortex in the depth of the CS at all AP positions and, with some scrutiny, can be followed at most locations to its termination on gyri encroaching the CS at the putative border between primary (functional area V1, Brodmann area 17) and secondary (functional area V2, Brodmann area 18) visual areas. The distinctive patterns of a higher $T_{2}{ }^{*}$ in the superficial gray matter layers than in the deeper layers, separated by the SoG, is visible everywhere in putative V1. Finally, it is interesting to note the $\mathrm{q}_{2}{ }^{*}$ contrast visible in parts of white matter, particularly the lower $T_{2}^{*}$ in parts close to the lateral ventricle, tentatively corresponding to large myelinated tracts. 


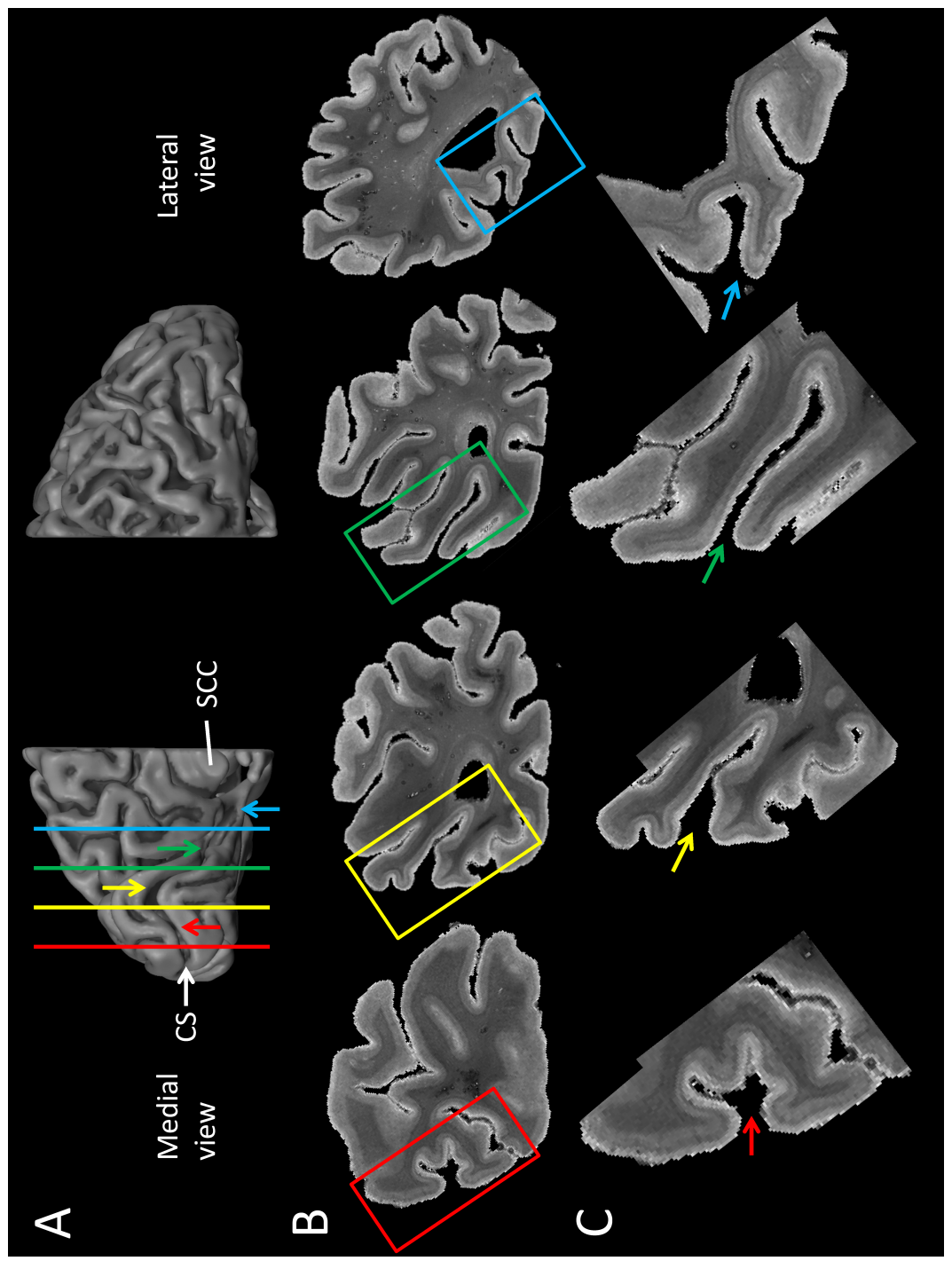

Figure 3.7: 3D surface reconstruction of entire left occipital lobe based on $200 \mu \mathrm{m} \mathrm{q} T_{2}^{*}$ maps (A) showing a medial view (left) and lateral view (right). (B) Coronal slices at four different anterior posterior positions along the calcarine sulcus, shown in different colours (C) and zoomed-in views of anatomical details along the same slices showing layer definitions and fiber tracts in white matter. CS: Calcarine sulcus (indicated by coloured arrows). SCC: Splenium of the Corpus Callosum. 


\subsection{Discussion}

We constructed a 16 channel cylindrical phased array receive coil to image a large post mortem occipital lobe sample $\left(\sim 80 \times 80 \times 80 \mathrm{~mm}^{3}\right)$ in a wide-bore 9.4T human scanner. The achieved SNR is strongly increased compared with a commercial human head coil at 7T. Although the transmit profile with a circularly polarized $(\mathrm{CP})$ transmit mode at $9.4 \mathrm{~T}$ is very inhomogeneous over the large sample, this challenge is successfully resolved with parallel transmit (pTx) using the kTpoints method. Increased spatial definition of cortical details is visible in $60 \mu \mathrm{m}$ anatomical images for the entire occipital lobe compared to lower resolutions. In addition, we were able to achieve sufficient control over SNR, $B_{0}$ and $B_{1}$ homogeneity and multi-contrast sampling to perform quantitative $T_{2}{ }^{*}$ imaging over the same volume at $200 \mu \mathrm{m}$.

\subsection{T large post mortem sample RF-coil}

The constructed dense, cylindrical 9.4T phased-array receive coil was targeted at acquiring high resolution anatomical and quantitative MRI of large post mortem samples. The coil's performance was evaluated through bench tests and ex vivo human occipital lobe imaging. The placement of the 2 rows of receive arrays covers the entire occipital lobe sample, but also ensures that the small diameter coils are as close as possible to the region of interest. This greatly enhanced their receive sensitivity and achieved SNR when compared to that of a larger loop $32 \mathrm{Ch}$ commercial head coil at 7T. The image SNR measured shows a 4-5 fold increase in maximum SNR (peripherally at the cortex) for the ex vivo sample coil over the commercial $7 \mathrm{~T}$ head coil. This can be attributed to a combination of higher field strength and smaller coil loops in close proximity to the sample, enabling much higher SNR in those areas. With the 7T head coil, only a few receive elements would couple with the sample (due to its size and positioning in the coil), thus reducing overall SNR in areas further away from coil elements. 
The position and placement of the coil elements in the 9.4T ex vivo sample coil allows for higher SNR throughout the sample although the gain are proportionally higher at the periphery, close to the coils, as is evident from Fig 3.4. For the occipital lobe sample this provided very high SNR specifically in most of the cortex and superficial white matter.

The presented ex vivo sample coil layout can of course be implemented at 7T, in which case it would also be expected to give significant gains for samples that fit its size over a commercial head coil. While the 7T field strength would yield more homogeneous $B_{0}$ and $B_{1}$ transmit fields than at 9.4T, it would also result in 33\% less overall SNR and lower $T_{2}{ }^{*}$ and other magnetic susceptibility moderated contrast, such as phase contrast and quantitative susceptibility imaging (QSI) [115, 125, 126]. The post mortem sample size of 80x80x80 $\mathrm{mm}^{3}$ targeted here would very difficult to accommodate in preclinical or animal MRI systems. Among current preclinical systems, only magnets with the widest bore diameter might be able to accommodate the sample using their largest diameter (and lowest performing) gradient and animal body RF coils. However, the imaging would likely be affected by reduced linearity in main $B_{0}$ field and gradient fields. Moreover, an insufficient receive channel count and clear bore space would likely be unable to support the customised 16 channel RF coil (along with a separate transmit coil) which provides most of the SNR gains reported.

The $B_{1}+$ transmit profiles in CP mode for 9.4T show the expected high central fields dropping off the periphery since the sample dimensions are close to the wavelength at 9.4T. In addition, there is asymmetry along the anterior-posterior direction with the transmit field dropping off to the anterior sample side in the acquired orientation. This is likely a result of the fact that the sample could not be aligned to the axial center ( $z$ direction) of the transmit coil, leaving a section of the anterior end of the sample outside of the transmit FoV where it underflips. However, this issue is largely rectified by using kT-points which results in a more homogeneous excitation profile along our region of interest, 
comparable to the excitation profile achieved at 7T with a single channel CP transmit coil. To ensure an even more homogeneous excitation profile along the entire length of the sample, future work could involve building a smaller diameter, sample-specific transmit coil with pTx capabilities at $9.4 \mathrm{~T}$.

\section{High resolution anatomical and quantitative MRI}

The high Rx signal and homogeneous Tx fields allowed us to acquire $100 \mu \mathrm{m}$ and $60 \mu \mathrm{m}$ isotropic $T_{2}{ }^{*}$ weighted data over the entire sample. While the SoG is clearly evident in both the $100 \mu \mathrm{m}$ and $60 \mu \mathrm{m}$ datasets, it is slightly better spatially defined in the $60 \mu \mathrm{m}$ scan. The higher contrast vessel wall and outer cortical boundary even more clearly show the better spatial definition which can be achieved. Although no spatial filtering was applied in the image reconstruction to present the quality of the raw data, a small degree of k-space filtering may be preferred in future neuroanatomy studies to mitigate a slight Gibbs ringing visible.

The $\mathrm{q} T_{2}{ }^{*}$ mapping of the entire human occipital lobe at $200 \mu \mathrm{m}$ isotropic shows some of the advantages of quantitative imaging. These include an even clearer definition of cortical architecture details, specifically here the SoG, inherent control of low frequency image inhomogeneity without a need to estimate receive coil sensitivity, and generally comparable images in quantitative physical units. The estimated $S_{0}$ shows a signal profile similar to the data absorbing information about the proton density as well as receive coil sensitivity. In our sample, $T_{2}{ }^{*}$ values in GM are greater than in WM and are also greater in the superficial GM layers than in the deeper layers. $T_{2}{ }^{*}$ relaxation time is inversely proportional to the concentration of myelin (and iron content), which is highest in WM and higher in the deeper layers of the GM. The calculated $T_{2}{ }^{*}$ values are higher than those acquired with in vivo subjects [124]. This could be partly explained by 
the hydration state of the tissue and the lack of active venous vasculature which would otherwise contribute to lower $T_{2}^{*}$. The estimated $T_{2}{ }^{*}$ are also comparable with previously reported $T_{2}{ }^{*}$ values in postmortem samples [127]. $T_{2}{ }^{*}$ estimates can be affected residual static $B_{0}$ inhomogeneity due to imperfect $B_{0}$ shimming [128]. This seems to be visible in Fig 3.6C where we notice slightly less contrast in a small area in the posterior-most section. Further improved iterative shimming routines could resolve this in the future. In addition, the rectified Rican noise floor can have an effect on the fitting of long TE low signal echoes. However, SNR was sufficiently high even in anterior parts of the sample, such that $T_{2}{ }^{*}$ could still be estimated, albeit with greater uncertainty. Moreover, MCMC sampling was performed with an offset-Gaussian likelihood function to account for the rectified noise floor.

The $200 \mu \mathrm{m} \mathrm{q} T_{2}^{*}$ imaging allows mapping of the SoG along the entire length of the calcarine sulcus. The SoG is clearly visible along the cortex in the depth of the CS at all AP positions in Fig 3.7 and can be followed at most locations to its termination at the border between primary (functional area V1, Brodmann area 17) and secondary (functional area V2, Brodmann area 18) visual cortex. This illustrates the capacity to map out and atlas architectural areas boundaries in future studies.

\section{Outlook}

Further future work can involve quantitative imaging of $T_{1}, T_{2}$, phase and magnetic susceptibility in addition to $T_{2}{ }^{*} . T_{1}$ imaging could be realized with relaxometry on mixing times in STEAM imaging or fitting of multiple flip angles in GRE imaging [95]. However, both would place an even higher demand on $B_{1}+$ homogeneity and control and quantification of absolute flip angles. Phase and quantitative susceptibility mapping (QSM) would require reconstruction, unwrapping and 
Chapter 3. High resolution anatomical and quantitative MRI of the entire human occipital lobe ex vivo at $9.4 T$

fitting of phase instead of magnitude data [97]. Quantitative MRI (qMRI) at resolutions higher than $200 \mu \mathrm{m}$, up to $100 \mu \mathrm{m}$ isotropic, may be possible using the current setup. In our experience, challenges here lie more in processing, analysing and visualizing the data than in the acquisition, as multi contrast $100 \mu \mathrm{m}$ acquisition could in principle be performed. qMRI at even higher resolutions up to $60 \mu \mathrm{m}$ isotropic might not be feasible due to insufficient SNR and multi contrast sampling leading to very long acquisition times. Diffusion MRI (dMRI) is another interesting quantitative technique to extend to large samples at very high resolutions. Post mortem dMRI has been performed previously over small FoV [129, 130, 131, 112, 105] and allows in situ validation by histology measures [132, 133, 134, 111, 135, 136, 137]. Diffusion MRI is inherently more SNR challenged than GRE imaging and benefits less from moving to UHF. In addition, it also requires high flip angle pulse sequences and good $B_{1}+$ homogeneity, making it a challenging prospect. Finally, the platform for UHF cortical layer specific quantitative MRI can be extended to even larger FoV allowing larger samples up to the entire post mortem human brain. 


\section{4}

\section{High resolution MRI neuroanatomy in intact human brains post mortem with a specialized 9.4T RF-coil}

Contents

4.1 Introduction . . . . . . . . . . . . . . . 95

4.2 Methods . . . . . . . . . . . . . . . . 97

4.3 Results . . . . . . . . . . . . . . . . . . . . . 103

4.4 Discussion . . . . . . . . . . . . . . . 113

Adapted from: Sengupta S., Fritz F. J., Caspers S., Zilles K., Lataster A., Herrler A., Tse D. H. Y., Poser B. A., and Roebroeck A. High resolution MRI neuroanatomy in intact humans brain post mortem with a specialized 9.4T RF-coil. (in prep) 



\subsection{Introduction}

Ex vivo imaging involves scanning post-mortem tissue over long scan sessions (several hours to a couple of days) at UHF which can yield ultra-high resolution datasets far below the millimetre scale while maintaining a sufficiently high Signal-to-Noise ratio (SNR) for the acquired images. Ex vivo UHF MRI to examine human brain tissue samples has been predominantly performed on preclinical or animal MRI systems [106, 107, 108, 109, 110, 111, 112, 113]. This takes advantage of the high field strength \& increased gradient performance of preclinical systems but is often limited to small human tissue samples (smaller than about $20 \times 20 \times 20 \mathrm{~mm}^{3}$ ), restricting size of the brain region examined. Alternatively, relatively small tissue samples have been investigated on a human 7T MRI system either using a commercial head RF coil or a small custom built RF coil [102, 101, 103, 114]. In parallel, a number of investigations have shown that several magnetic resonance imaging (MRI) contrasts (such as $T_{1}, T_{2}, T_{2}^{*}$ and phase) show sensitivity to iron and, especially, myelin content in gray matter in vivo [89, 90, 91, 92, 93, 94, 95, 96] which has ignited ambitions of MRI-based in vivo histology [92, 97, 93, 98, 139]. In some of these investigations quantitative MRI, providing quantitative contrasts in physical units rather than $T_{1}$ or $T_{2}^{*}$ weighted acquisitions, has been shown to have clear advantages for cortical MRI based histology [92, 97, 99]. Therefore, extending ex vivo UHF studies to intact human brains, while maintaining high resolution and the potential for quantitative imaging, would be an important step. It would allow investigations of the architecture of several cortical areas over their entire extent and their complete borders where architecture changes, which is very relevant to the translation to the in vivo situation. 
The investigation of intact human brains post mortem with large bore systems can achieve resolution considerably superior to that achievable in-vivo [140, 141, 142, 143, 144]. However, the achievable resolutions and contrast are limited compared to small sample studies by gradient performance, non-optimized RF-coils, and RF-field inhomogeneity over the brain at high main field strengths $(\geq 7 \mathrm{~T})$. With increases in main field strengths, from $3 \mathrm{~T}$ to $7 \mathrm{~T}$ and beyond, and increases in the number of channels in phased-array RF coils, it has become increasingly clear that RF coils specifically built to subject or sample size and shape can offer very large advantages [75, 48, 88, 68]. Although this has specially been true for human in-vivo $7 \mathrm{~T}$ coil designs, this approach is less developed in the field of ex-vivo tissue MR. Investigations of intact human brains ex vivo in particular have therefore been limited the contrast and Signal-to-Noise ratio that can be achieved with a non-specialized setup, such as an in-vivo head coil. Therefore, in this study, we focus on a custom built UHF RF-coil as a key technology for high resolution ex vivo anatomical and quantitative MRI for the intact human brain. We designed and characterised a conformal whole post mortem human brains coil for use in a large-bore 9.4T system and compared its performance with that of a standard whole head array coil at 7T. The two specific aims in enabling brain atlassing and cortical laminar discrimination over the entire brain are the following. First, we aim to achieve sufficient control over $B_{1}$ homogeneity with parallel transmit techniques to generate high resolution anatomical images (at $100 \mu \mathrm{m})$ for the entire human brain. Second, we aim to achieve multi-contrast sampling to perform quantitative $T_{2}^{*}$ imaging over the enitre brain at $200 \mu \mathrm{m}$. Third, we evaluate the potential of $100 \mu \mathrm{m}$ data and $200 \mu \mathrm{m}$ quantitative data for anatomical atlassing of sub cortical structures and cortical laminar discrimination. 


\subsection{Methods}

\section{RF-coil and sample container construction}

A 3D conformal sample container for post-mortem human brains was first designed in SolidWorks using anatomical MRI data. The mesh file thus generated was then surface-rendered and extruded by $2 \mathrm{~mm}$ in all directions and modelled into a volumetric container in 2 halves. The container was then 3D printed using a watertight, chemically resistant and susceptibility matched material (Fig 4.1A). (Somos Watershed XC11122, DSM, Heerlen). The custom-built RF coil (Life Services LLC, Minneapolis, MN) consists of 2 parts: a conformal receive former modelled as a precise fit around the $3 \mathrm{D}$ printed sample container while two halves of a hollow, ellipsoidal shell were used to accommodate the transmit coils (Fig 4.1B-C).

Twenty four receive coils were laid out in a phased-array configuration on the receive former, with 14 elements covering the superior part of the sample (coil's inferior side) and 10 elements for the inferior end of the sample (coil's superior side). The receive coils ranged between 5 and $7 \mathrm{~cm}$ in diameter, ensuring full coverage of the sample (Fig 4.1D). On-coil preamplifiers with active detuning, tuning \& matching and balun circuitry were implemented on all coils, while ensuring that the preamplifiers were aligned along the $\mathrm{z}$-axis to reduce Hall effects. Each individual receive loop - constructed using $1 \mathrm{~mm}$ thick, polyimide coated copper wires - was decoupled from each other using a combination of optimal geometric overlapping ( $20 \%$ coil diameter) and preamplifier decoupling. Cable traps were also implemented - by tailoring a solenoid using the receive coaxial cable around a polycarbonate screw, and bridging a capacitor across it - in order to reduce common-mode current effects. (Fig 4.1E)

The 8 transmit channels were laid out in a $2 \times 2$ matrix, with 4 loops on each half of the coil. The loops were $7 \mathrm{~cm}$ in diameter and constructed 
using $2 \mathrm{~mm}$ thick silver-coated copper wires with 3 distributed capacitors on each coil, and spaced apart from each other in order to provide extended, full 3D parallel transmission and to reduce coupling between adjacent channels at the same time. A semi-circular slotted RF shield was placed on both sides of the coil build to reduce eddy current losses. All transmit and receive channels were tuned and matched to $400 \mathrm{MHz}$ at $50 \Omega$.

\section{RF coil bench measurements}

Bench tests were performed using a Agilent HP E5071C ENA Series network analyser, a customized coil-plug bed and a post-mortem whole human brain in the sample container as load. Measurements towards quantifying receive coil decoupling and preamplifier decoupling for each coil element were performed. The bench tests were undertaken to validate coil tuning and matching, preamplifier decoupling for each coil element and active coil detuning.

Each loop on the receive array was tuned and matched to $400 \mathrm{MHz}$ at $50 \Omega$. The coupling between neighbouring elements was measured using $S_{21}$ measurements - by connecting the coil outputs to the network analyser while the other coil elements were detuned. In this way, the critical overlap between elements could be further optimised, ensuring minimal mutual inductance. Preamplifier decoupling of a single loop was measured as the difference in $S_{21}$ measurement using a pair of decoupled double-loop probes, first with the coil power matched to $50 \Omega$ under load but without the preamplifier present and then with the coil terminated using the low input impedance preamplifier, while keeping all other coils in the receive array detuned. The active detuning for each receive element was also measured as the difference in an $S_{21}$ measurement, between when the coil is matched to $50 \Omega$ and when detuned. 


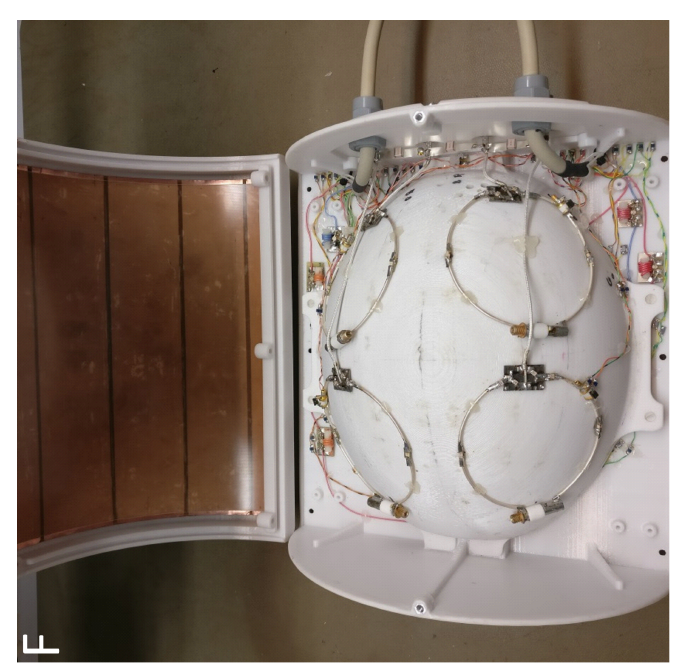

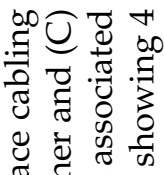

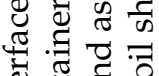

क्ष

. ठ ठ

.

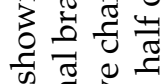

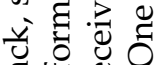

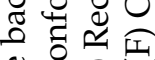

\& 8 王

¿ $e^{\infty}$

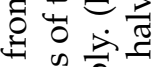
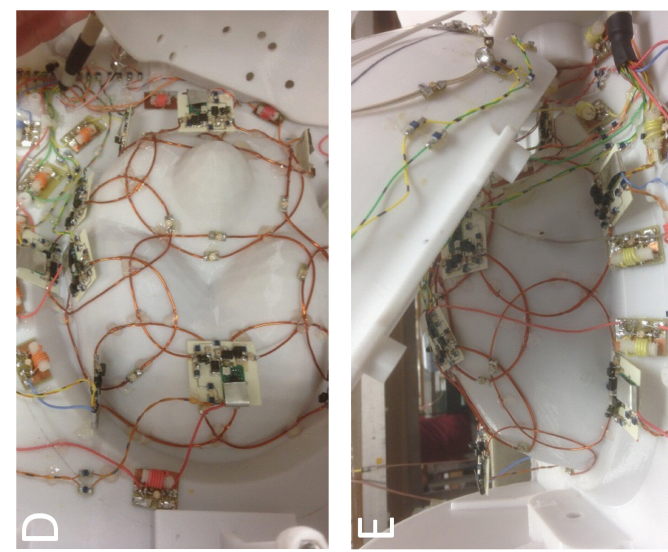

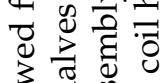

3 조 की

하융

क ष

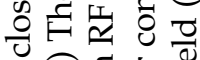

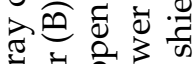

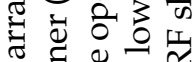

-

竞 00.0

ठ $\cong$ क क

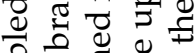

हี

की
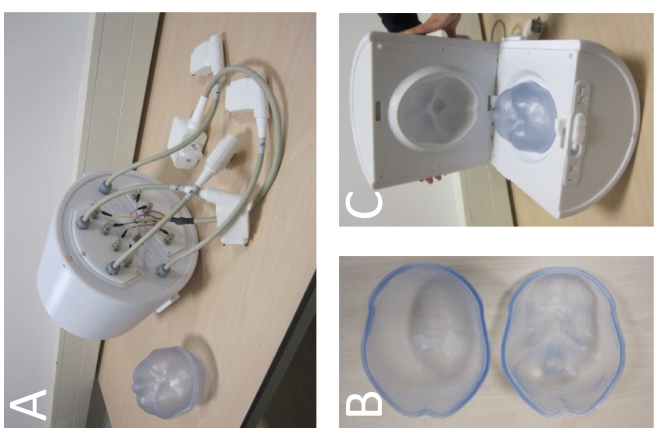

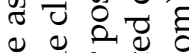

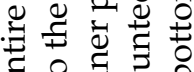

더유 응

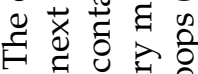

《

工

$\because$ 드음 항 몽

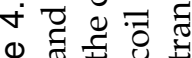

흠 
For the 8 channel transmit array, a similar method of assessment was adopted. Each loop of the transmit channel was tuned and matched to $400 \mathrm{MHz}$ and $50 \Omega$ respectively using an $S_{11}$ measurement, with the coil connected directly to the network analyser while being loaded with the post-mortem whole brain sample. $S_{21}$ measurements were used to determine the coupling between the 4 adjacent coil elements on the same side. Active detuning was measured as an $S_{21}$ measurement using a pair of decoupled pickup probes lightly coupled to the coil under investigation, and forward biasing the diode in order to detune the coil. The coil under test was terminated to $50 \Omega$ during this measurement, while keeping the other 3 loops in the detuned state.

\section{Sample preparation}

Tissue used for imaging was obtained post mortem from a male subject without any known neurological or psychiatric disorders. The tissue donor gave his informed and written consent to the donation of his body for teaching and research purposes regulated by the Dutch law for the use of cadavers for scientific research and education. A handwritten and signed codicil from the donor, posed when still alive and well, is kept at the Department of Anatomy and Embryology Faculty of Health, Medicine and Life Sciences, Maastricht University, Maastricht, The Netherlands. The formalin-fixed whole brain sample was first immersed in 6 times its volume of phosphate-buffered solution (PBS) for 6 weeks to wash out the formalin and rehydrate the sample. After washing, the sample was placed in the 3D printed conformal sample container and embedded in a solution of PBS mixed with $1 \%$ $\mathrm{NaN}_{3}$ (sodium azide) for imaging. 


\section{MRI acquisition and analysis}

Experiments were performed on a 9.4T, $830 \mathrm{~mm}$ bore human MR scanner (Magnetom 9.4T, Siemens Medical Solutions, Erlangen, Germany) equipped with an $80 \mathrm{mT} / \mathrm{m}, 330 \mathrm{~T} / \mathrm{m} / \mathrm{s}$ head gradient system. To overcome the $B_{1}^{+}$inhomogeneity that is severely inherent in UHF, it was necessary to use pTx composite pulses following the pipeline defined in [120]. For the pulse design, a main field profile $\left(B_{0}\right)$ was acquired using the fieldmap sequence [117] and transmit profile $\left(B_{1}^{+}\right)$maps from each of the transmit channels was acquired with a $T_{2}^{*}$ compensated version of DREAM [118]. A full pTx, kT-points with 8 composite subpulses [121] was calculated and subsequently optimized for $B_{1}^{+}$homogeneity with $B_{0}$ compensation [119]. To effectively quantify the improvement in $B_{1}^{+}$excitation profiles when using the optimized composite pulse in comparison with a circularly polarized single pulse, $B_{1}^{+}$ images using both modalities were acquired using the AFI sequence [80]. With the obtained composite pulse, anatomical whole brain image at high resolution was acquired using a 3D single echo kT-GRE acquisition and for $q T_{2}^{*}$ characterization of the same specimen, a multiecho 3D kT-GRE acquisition was used. The sequence parameters used for both acquisitions are summarized in table 4.1.

The in-plane sagittal field of view for all the experiments was $162 \times 153$ $\mathrm{mm}^{2}$ at EPI factor 1, one average. For all the acquisitions, a $0 \mathrm{~V}$ noise reference scan was also acquired matching protocol parameters (e.g. bandwidth, repetition time, echo times and base resolution). A noise covariance corrected root of sum of squares (cov-rSoS) [122] offline reconstruction was performed using home-made programs in MATLAB on the acquired data. For $q T_{2}^{*}$ estimation from the $q T_{2}^{*}$-weighted GRE images were analysed in a voxel-based analysis approach using the Maastricht Diffusion Toolkit (MDT) [123] by fitting echo decay curves per voxel. Powell optimization with patience 5 and double precision was employed for $q T_{2}^{*}$ estimation, in where unweighted signal $\left(S_{0}\right)$ was initialized using the lowest echo and an offset-Gaussian likelihood 
Chapter 4. High resolution MRI neuroanatomy in intact human brains post mortem with a specialized 9.4T RF-coil

\begin{tabular}{|c|c|c|c|c|c|c|}
\hline Type & TR (ms) & $\begin{array}{c}\text { Echo } \\
\text { time } \\
(\mathrm{ms})\end{array}$ & $\begin{array}{c}\text { BW } \\
(\mathrm{Hz} / \mathrm{px})\end{array}$ & $\begin{array}{c}\text { Flip } \\
\text { angle } \\
(\mathrm{deg})\end{array}$ & $\begin{array}{c}\text { Matrix } \\
\text { dimen- } \\
\text { sions } \\
(\mathrm{px})\end{array}$ & $\begin{array}{c}\text { Time } \\
(\mathrm{h}: \mathrm{m}: \mathrm{s})\end{array}$ \\
\hline $\mathrm{q} T_{2}^{*}$ & 43 & 8,14, & 120 & 28 & $\begin{array}{c}810 \mathrm{x} \\
768 \mathrm{x}\end{array}$ & $10: 08: 30$ \\
$200 \mu \mathrm{m}$ & & 24,33 & & & 704 & \\
\hline $100 \mu \mathrm{m}$ & 48 & 22 & 30 & 29 & $1620 \mathrm{x}$ & $22: 37: 03$ \\
$\mathrm{GRE}$ & & & & & $\begin{array}{c}1534 \mathrm{x} \\
1408\end{array}$ \\
anat. & & & & & $320 \mathrm{x}$ & $00: 34: 15$ \\
\hline $600 \mu \mathrm{m}$ & 40 & 5,15, & 120 & 30 & $259 \mathrm{x}$ & \\
GRE & & 24,34 & & & 256 & \\
anat. & & & & & & \\
\hline
\end{tabular}

Table 4.1: Sequence parameters for 3 different acquisitions

function to account for the Rician rectified noise floor.

Signal-to-noise ratio (SNR) comparison using a 7T Siemens MAGNETOM scanner (Siemens Healthcare, Erlangen, Germany) with the Nova Medical 32-channel Head Coil (Nova Medical Inc., MA, USA) was also performed. The excitation profiles from both 7T and 9.4T measurements were acquired using the TurboFLASH readout (TFL) sequence [145] in circular polarized (CP) mode and the AFI sequence in CP mode and in pTX mode using kT-points, respectively. A GRE using monopolar-RO at $600 \mu \mathrm{m}$ isotropic resolution was used in both scanners under the same protocol parameters, with a matched zero volt noise acquisition. The pseudo-replica approach [83, 82] was implemented in calculating the image SNR for both coils using the subsequent noise data. A 100 replicas were generated by adding random samples from the noise scan onto the GRE k-space of the standard image acquisition prior to Fast Fourier Transform (FFT), while maintaining the noise covariance between the independent receive channels. The final image SNR was then calculated as the ratio of the mean of the image to the standard deviation of the noise over these 100 replicas. 
We also acquired noise-only scans and fully sampled 3D GRE for evaluation of the parallel imaging performance of the whole brain coils. Scans were performed using a 3D GRE sequence with the following parameters: Echo Time $(\mathrm{TE})=2.64 \mathrm{~ms}$; Repetition Time $(\mathrm{TR})=30 \mathrm{~ms}$; FoV $=256 \times 256 \mathrm{~mm}^{2} ; 1 \mathrm{~mm}$ isotropic; the noise scan was acquired with fewer $K_{Y}$ and $K_{Z}$ encode and by turning the transmitter voltage to zero. The GRE scan was Fourier-interpolated to a matrix size of [120 $x 120 \times 120$ ] in order to allow emulation of different SENSE undersampling factors on the bases of data set $(R=2,3,4)$. The G-factor maps and SENSE reconstructions were performed using MATLAB. For the $\mathrm{g}$-factor maps we used the formulation given in the original SENSE paper [18] where $\Psi$ is the covariance matrix. Both g-factors and corresponding SENSE reconstructions were performed at undersampling factors between 2 and 4 , by sub-sampling the full k-space data set that had been re-sampled to [ $120 \times 120 \times 120]$. The reconstruction itself was a SENSE reconstruction as described in [18].

\subsection{Results}

\section{RF coil characterization}

All receive elements were matched between $-21 \mathrm{~dB}$ and $-28 \mathrm{~dB}$ for the for the whole brain post-mortem sample at $400 \mathrm{MHz}$. The active PIN diode detuning provided an isolation of $24 \mathrm{~dB}$ or better between tuned and detuned states. The decoupling between neighbouring, overlapping receive elements ranged between $-10 \mathrm{~dB}$ to $-21 \mathrm{~dB}$. Decoupling between next-nearest neighbouring elements (or non-overlapping elements) of the array ranged between $-18 \mathrm{~dB}$ to $-26 \mathrm{~dB}$. An additional $18 \mathrm{~dB}$ of isolation was achieved through preamplifier decoupling. The decoupling between pairs of adjacent transmit elements was $-11 \mathrm{~dB}$ or lower. Active pin-diode detuning of each transmit loop ranged between $-17 \mathrm{~dB}$ to $-20 \mathrm{~dB}$. 
Figure 4.2 shows the receive characteristics of the whole brain coil. Fig $4.2 \mathrm{~A}$ shows the noise correlation matrix with the correlations ranging between $0.2 \%$ and $78 \%$ with an average of $20 \%$. Fig $4.2 \mathrm{~B}$ shows the channel-combined reconstructions for a $200 \mu \mathrm{m}$ isotropic GRE acquisition along a coronal slice using a root Sum-of-squares (rSoS) channel combination (left) and noise covariance weighted root Sum-of-squares (cov-rSoS) channel combination (right). Relatively even coverage in the periphery and substantial receive penetration to image the entire sample can be seen, especially when using a noise covariance weighted reconstruction. Figure 4.3 shows the comparison between the transmit profiles when using a single channel circularly polarised $(\mathrm{CP})$ mode against a parallel transmit (pTx) mode utilising the kT-points method at 9.4T. The CP excitation profile shows very low transmit homogeneity with several areas severely under-flipped. This leads to severe drop-outs, and SNR and contrast variations over the whole brain sample. The use of the kT-points method at 9.4T greatly increases the homogeneity of the excitation profile over the sample. This can be seen to result in the recovery of the dropout signal and highly homogenous contrast across the sample.

To quantify the signal-to-noise performance of the custom-built whole brain coil, we compared it to a commercial 7T in vivo head-coil. Fig 4.4A shows the SNR comparisons for the same ex-vivo sample across $7 \mathrm{~T}$ and $9.4 \mathrm{~T}$ for a $600 \mu \mathrm{m}$ isotropic proton-weighted GRE acquisition. As is evident, the SNR with the whole brain ex-vivo coil at 9.4T is substantially higher across the entire imaging volume by about a factor of 3 in the center and by a factor of 5 at the periphery of the brain. This mostly reflects a coil geometry advantage consisting of Rx coils which are closer and which cover the complete surface of the sample. Superior brain tissue SNR is highest in both coils due to the orientation of the sample within the coil, as the superior side of the brain lies facing downwards close to the receive loops at the bottom of the coil. In the case of the whole brain coil SNR at other extremes of the brain (such as inferior, posterior and anterior) is also increased with respect to the center, because of close Rx coil coverage there. 


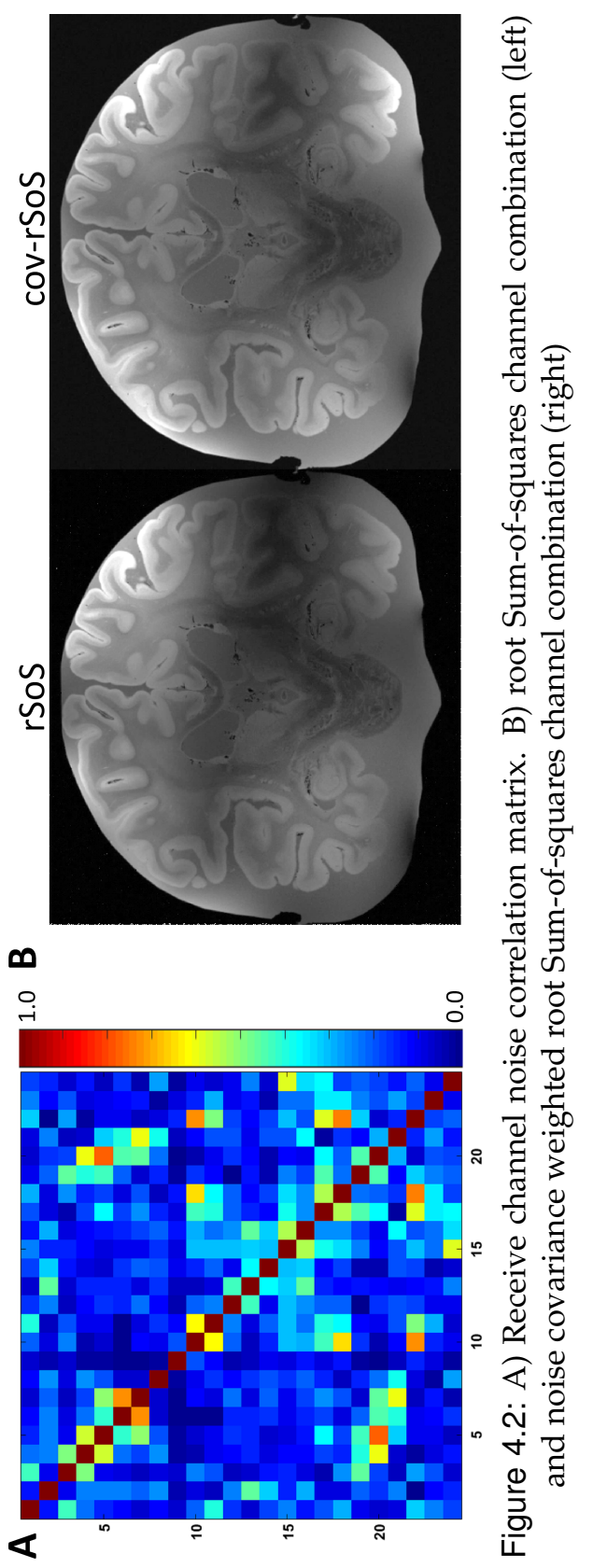




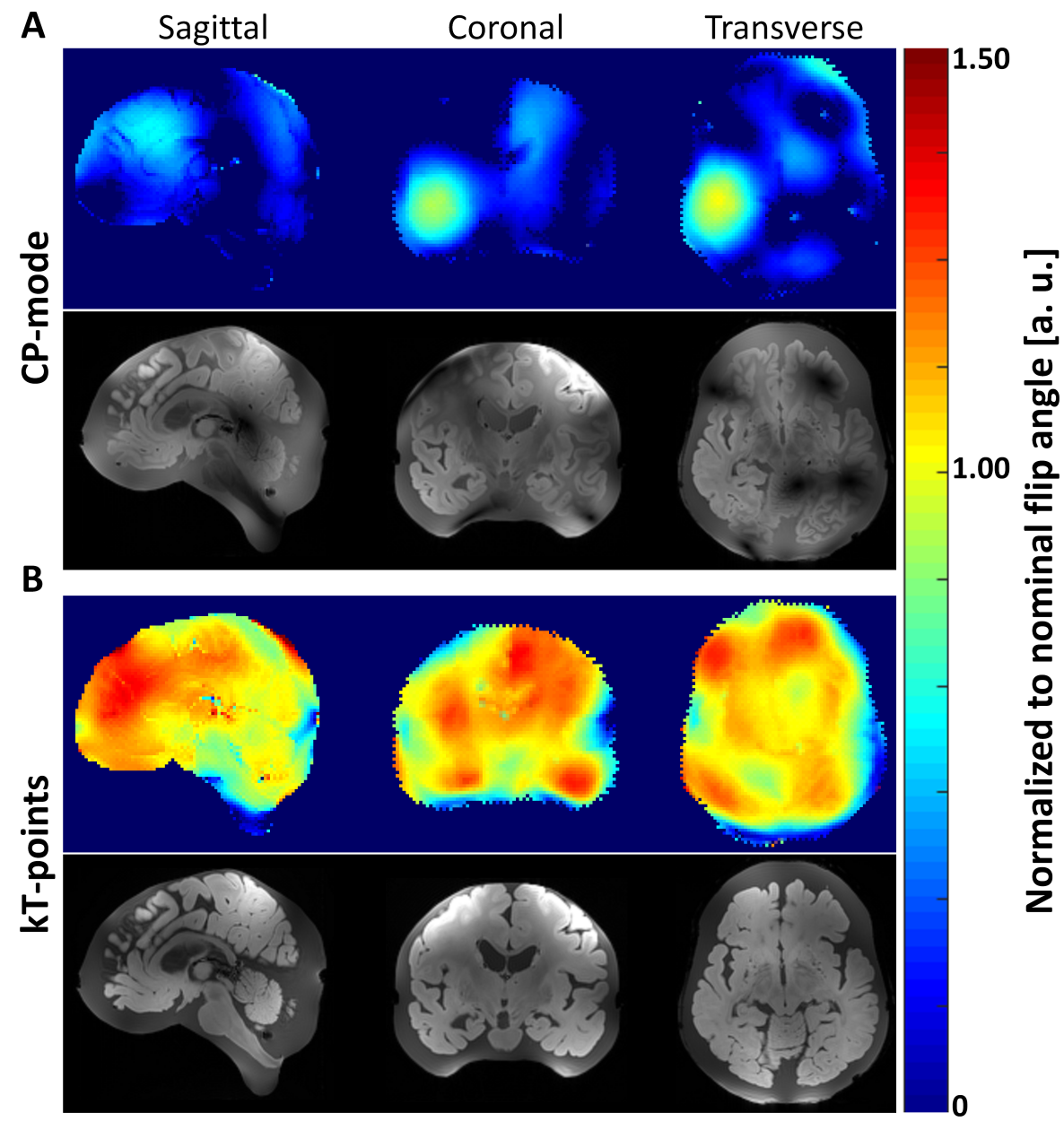

Figure 4.3: Transmit $\left(B_{1}^{+}\right)$profile maps, normalized to nominal flip angle $(=1.0)$, and corresponding GRE acquisitions of the 9.4T circularly polarised (CP) mode excitation (A) and 9.4T kT-points homogenized excitation (B) across sagittal, coronal and transverse planes 
A
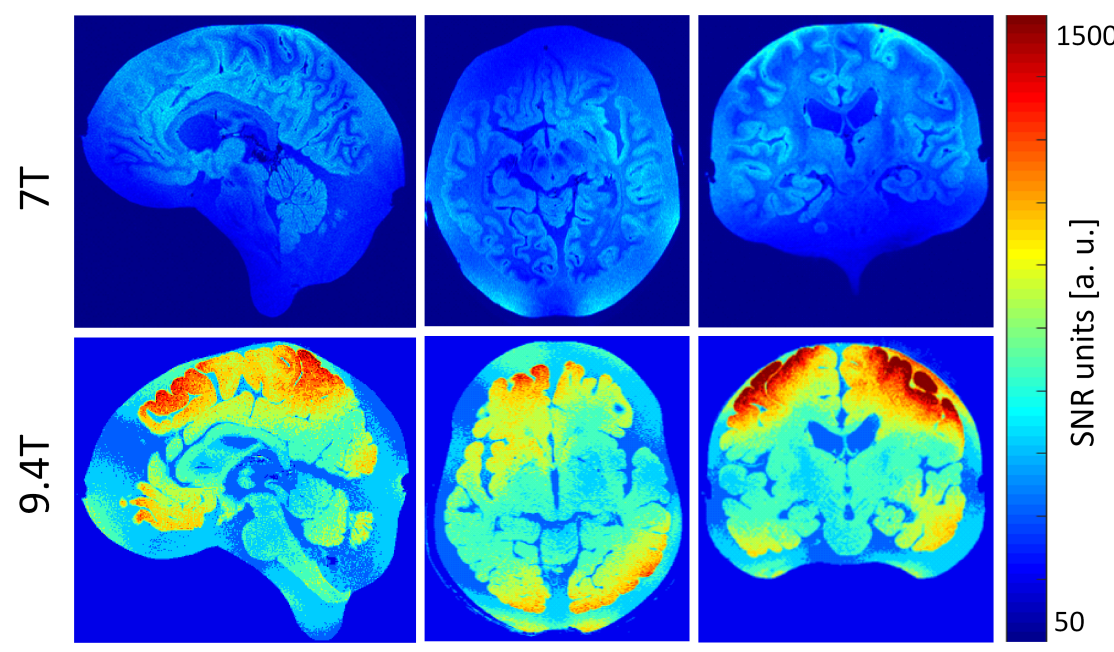

B
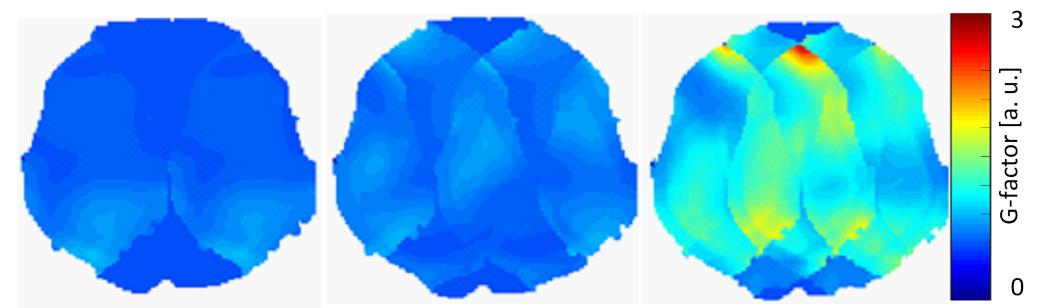

$\mathbf{R}=\mathbf{2}$

$R=3$

$$
R=4
$$
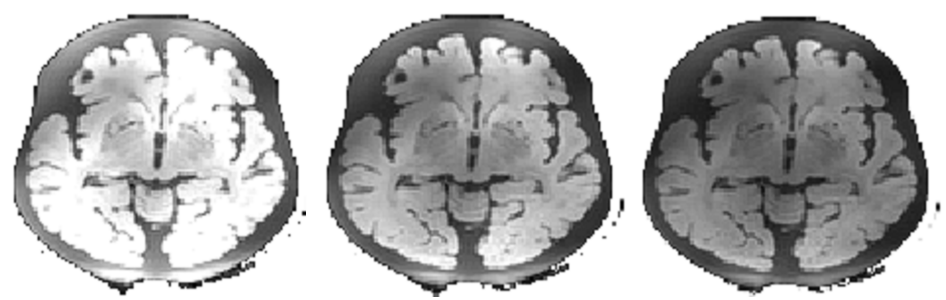

Figure 4.4: (A) SNR maps for the 7T Nova coil (top row) and the whole brain coil at $9.4 \mathrm{~T}$ using the same post-mortem sample (4A, bottom row) for a $600 \mu \mathrm{m}$ proton-density weighted GRE acquisition. (B) Transverse gfactor maps for the whole brain coil for L-R acceleration (top row) and corresponding SENSE reconstructions for acceleration factors $R=2,3,4$ 
We also quantified the degree to which SNR can be maintained under accelerated imaging. Fig 4.4B shows the local g-factor maps generated for the whole brain coil in the transverse plane for 1D acceleration factors in the left-right direction and their reconstructions in SNR units. At acceleration factor $\mathrm{R}=4$, the maximum g-factor is 2.8 , with much lower g-factors below 1.2 exhibited for lower acceleration factors of $\mathrm{R}=2,3$. This shows accelerated imaging with $\mathrm{R}=2$ or $\mathrm{R}=3$ is feasible without excessive noise amplification, as shown by the SNR-unit images which are dominated in these cases by the 1 /sqrt factor.

The high quality GRE images produced by the whole brain coil allow quantitative MRI relaxometry over the entire post-mortem human brain. Fig $4.5 \mathrm{~A}$ shows $200 \mu \mathrm{m}$ isotropic whole brain multi-echo GRE imaging at echo times varying from $\sim 9 \mathrm{~ms}$ to $\sim 35 \mathrm{~ms}$. This data is suitable for quantitative $T_{2}^{*}\left(q T_{2}^{*}\right)$ mapping, as shown in Fig 4.5B. A high quality $q T_{2}^{*}$ map results from a non-linear exponential fitting of the multi-echo data. The $q T_{2}^{*}$ volume provides quantitative contrast, with white matter (WM) $T_{2}^{*}$ values of $10-12 \mathrm{~ms}$ and gray matter (GM) $T_{2}^{*}$ values of $20-25 \mathrm{~ms}$. In addition, the quantitative imaging has the advantage of removing transmit and receive related bias fields, which are absorbed into the $S_{0}$ map.

To assess the potential to create 3D MRI-based anatomical atlasses, Fig 4.6 shows labelling of deep anatomical structures in a coronal slice in a $100 \mu \mathrm{m}$ (non-quantitative) $T_{2}^{*}$ weighted $\left(T_{2}^{*} \mathrm{w}\right)$ volume and a $200 \mu \mathrm{m}$ $q T_{2}^{*}$ volume. These two, roughly acquisition time matched, volumes show the trade-off between superior resolution (in the $100 \mu \mathrm{m} \mathrm{T} T_{2}^{*} \mathrm{~W}$ volume) and superior SNR and homogenous contrast (in the $200 \mu \mathrm{m} q T_{2}^{*}$ volume). Many subcortical anatomical structures can be distinguished based on the $T_{2}^{*}$ contrast.

Figure 4.7 shows characterization of cortical layering in a coronal slice through the calcarine sulcus in a $100 \mu \mathrm{m}$ (non-quantitative) $T_{2}^{*}$ weighted $\left(T_{2}^{*} \mathrm{w}\right)$ volume and a $200 \mu \mathrm{m} q T_{2}^{*}$ volume. Clear separation of white and gray matter is seen in the enlarged images on the right, 
which also highlight the stria of Gennari in primary visual area V1. Further sub-layering is also distinguishable in places. In addition, the pronounced $T_{2}^{*}$ contrast between more myelinated infragranular layers and less myelinated supra-granular layers stands out, especially in the $q T_{2}^{*}$ map. 
Chapter 4. High resolution MRI neuroanatomy in intact human brains post mortem with a specialized 9.4T RF-coil

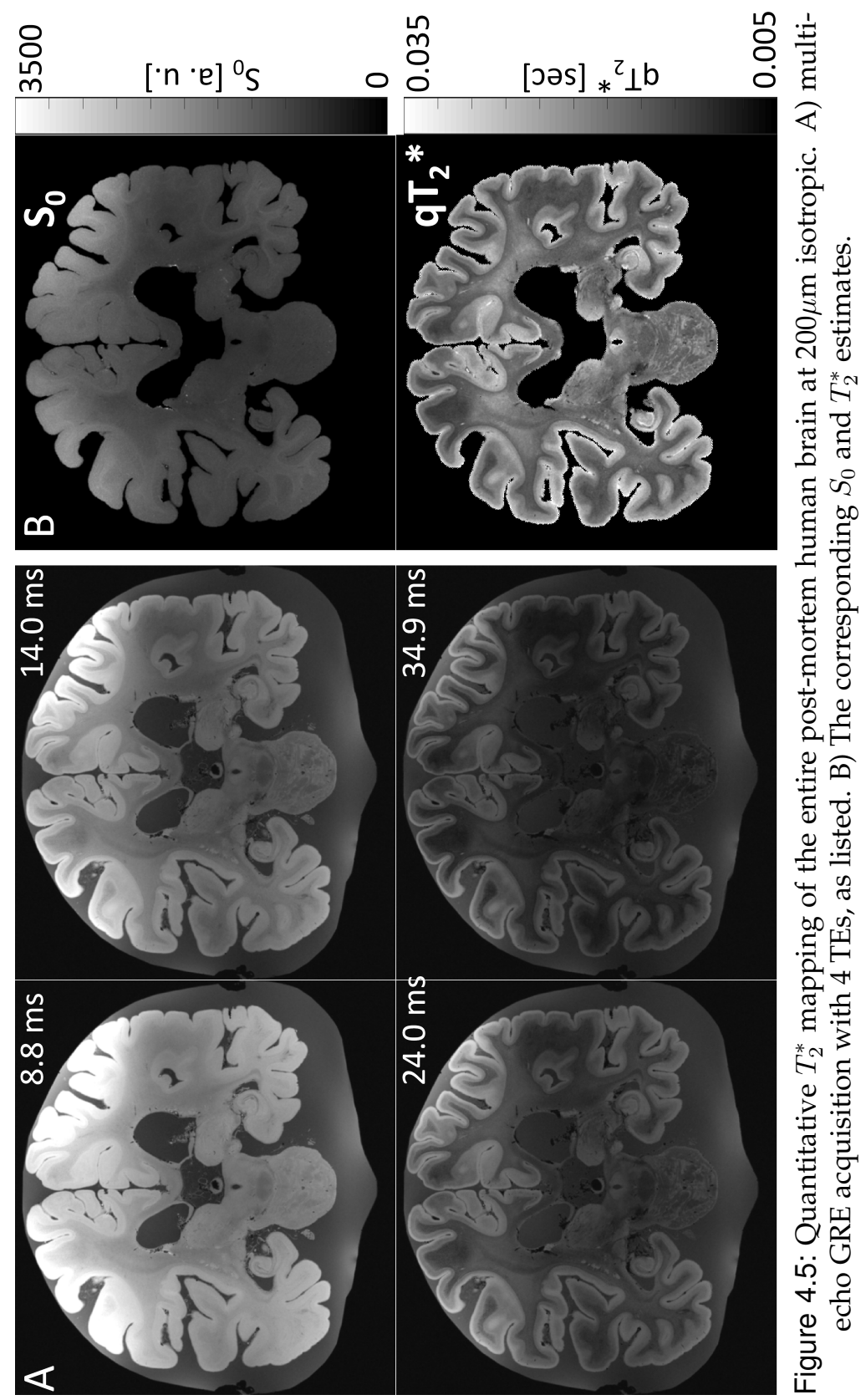




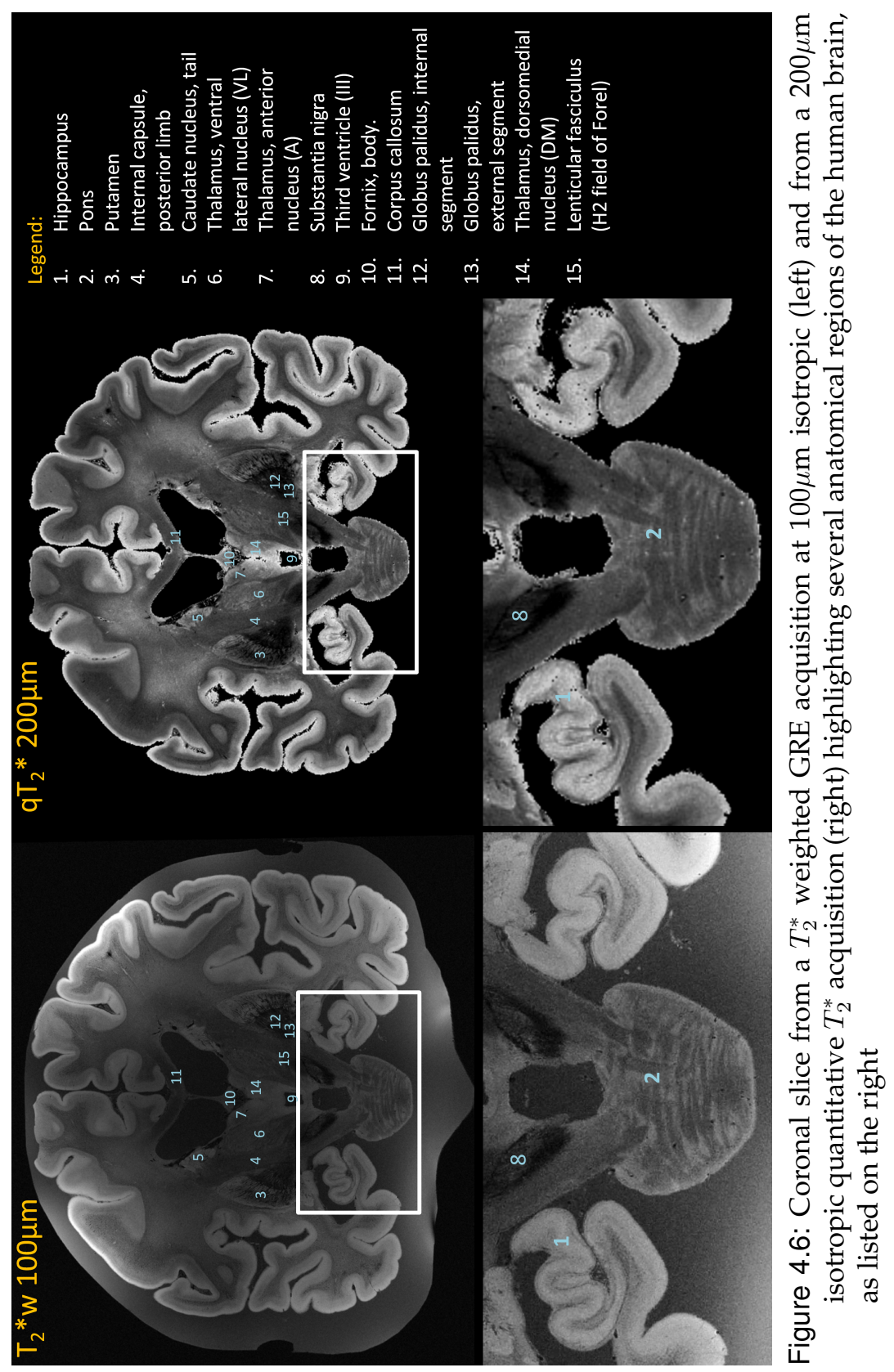


Chapter 4. High resolution MRI neuroanatomy in intact human brains post mortem with a specialized 9.4T RF-coil

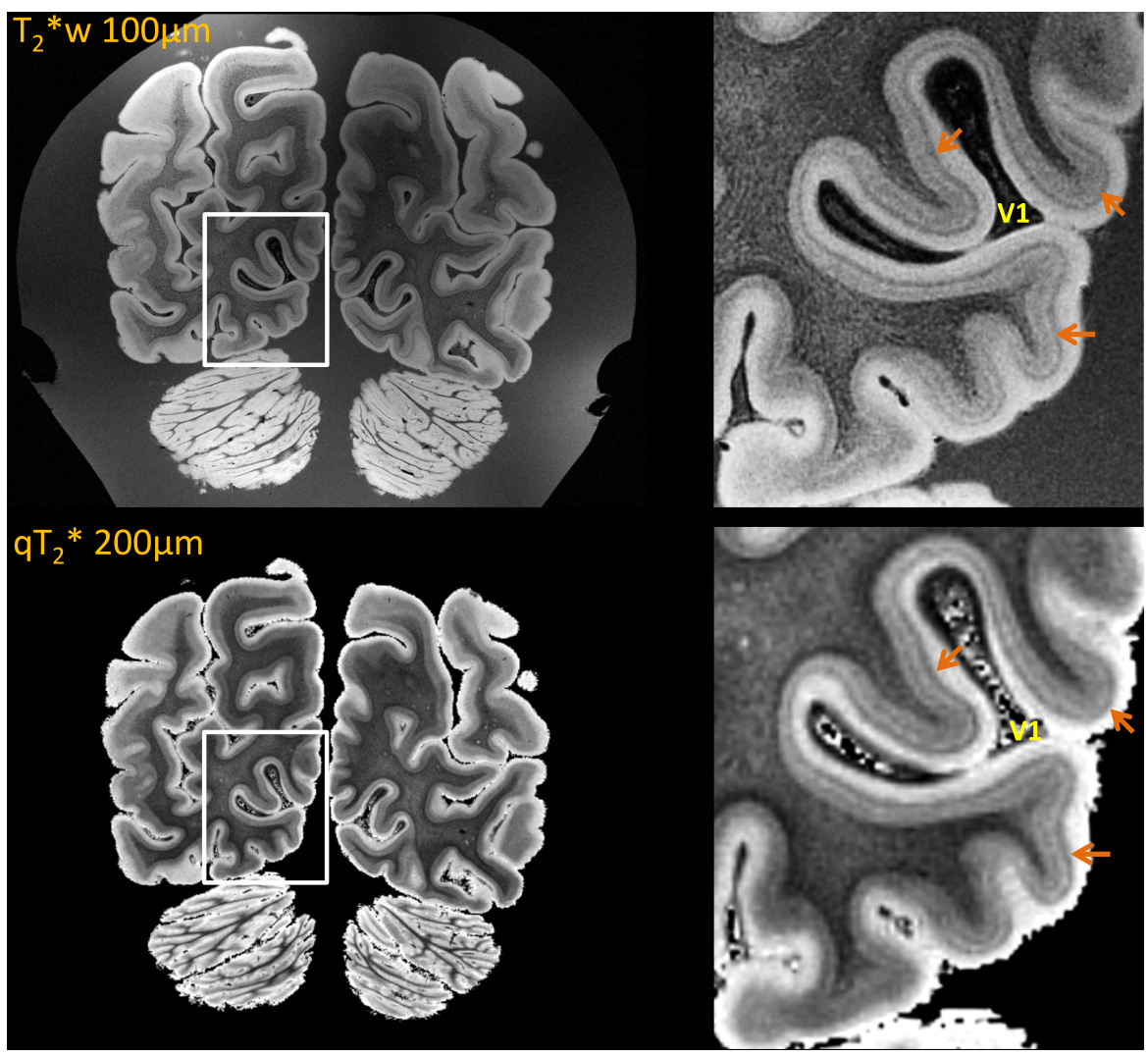

Figure 4.7: Coronal slice from a $T_{2}^{*}$ weighted GRE acquisition at $100 \mu \mathrm{m}$ isotropic (top) and from a $200 \mu \mathrm{m}$ isotropic quantitative $T_{2}^{*}$ acquisition (bottom) showing increased layer definitions in visual area V1 


\subsection{Discussion}

A 24 channel receive / 8 channel transmit RF coil was constructed to image intact human brain samples in a wide-bore 9.4T human scanner post mortem. The SNR realised with the whole brain coil is considerably higher compared to a human head coil at 7T. Inhomogeneous transmit profiles resulting from a circularly-polarised (CP) mode at 9.4T were successfully mitigated by the use of parallel transmit (pTx) using the kT-points method. The high SNR achieved allowed for increased spatial definition, with cortical details clearly visible in the $100 \mu \mathrm{m}-200 \mu \mathrm{m}$ isotropic resolution anatomical images. Having acquired sufficient control over $B_{0}$ and $B_{1}$ homogeneity, SNR and multicontrast sampling, we were also able to perform quantitative $T_{2}^{*}$ imaging over the entire volume.

\subsection{T whole brain post mortem sample RF-coil}

The conformal, 24 channel Rx/ 8 channel Tx phased array coil was constructed with the sole purpose of undertaking high resolution anatomical and quantitative MRI acquisitions of whole brain post-mortem samples. Once built, the coil performance was evaluated through bench tests and ex-vivo sample scanning. The receive array was constructed on a conformal former ensuring that the receive loops are in close proximity to the sample / region of interest, much closer than would be possibly in a commercially available in-vivo head coil. This further enhances receive sensitivity and the resulting SNR when compared to that of the head coil at 7T, even though it uses loops of a similar diameter. The measured image SNR shows a five-fold increase in maximum SNR at the periphery (at the cortex) and a three-fold increase in the center for the whole brain coil over the compared head coil at 7T. This is a result of conformality and proximity of the coils with respect to the sample, enabling higher SNR, and the higher 9.4T field strength. This isn't the case with the whole head coil at 7T, as a 
number of the receive elements would be further away from the sample, resulting in inefficient coupling with the sample and thus reducing the overall available SNR in areas that lie at a distance from coil elements. While the position and placement of receive elements in the 9.4T whole brain coil allows for overall higher SNR throughout the sample, the gain is proportionally higher at the periphery, close to the coils, as is evident in Fig 4.4A, resulting in very high SNR in the cortical and superficial white matter areas. It must be noted however, that the SNR comparison across field strengths is still partially non-absolute. This is because of longer $T_{2}^{*}$ and shorter $T_{1}$ at $7 \mathrm{~T}$ in comparison to $9.4 \mathrm{~T}$, such that an SNR measurement with matched TR and TE parameters tends to overestimate SNR at 7T.

While overall receive channel noise correlations are low, an issue of note is the rather high noise correlation values between certain individual coil pairs. Given the non-standard geometry of the receive former, it was not possible to implement the optimal overlap (0.2 0.25 times coil diameter) across certain coil pairs, resulting in reduced decoupling and higher noise correlation between said channels. The higher noise correlation can also be attributed to the shared resistance across said channels acquired through the sample. However, the effects of increased noise correlations were alleviated in the data by using cov-rSoS reconstructions. The coil's overall receive performance could perhaps be further enhanced by increasing the number of receive channels from 24 to 32, which would result in smaller, individual loops and therefore increased SNR in areas close to the coils. However, this would only result in SNR increase in the center of the brain in the case of a well decoupled array, such as can be achieved on a spherical or cylindrical former. Given the conformal (irregular convex and concave) shape especially on the inferior surface of the brain, such decoupling (and the optimal coil overlap) would be difficult to achieve for a very large element count. The 24 receive loop design also already supported accelerated imaging in a single phase encode direction up to a factor of 3 , showing highly independent spatial selectivity of the loops. Additionally, the 8 additional receive channels available 
on a common 32 channel MRI system are left free in the current design for useful peripheral data collection, such as online field monitoring [146].

Given that image acquisition at very high resolutions can be time consuming, accelerated imaging is often undertaken in order to reduce acquisition time at the cost of SNR. The SNR costs of accelerated imaging is exacerbated by high g-factors resulting from coil geometry. However, at acceleration factors up to 3, the calculated g-factors for the whole brain coil are very low. This, coupled with the intrinsically high SNR capability of the coil, can allow for trade-offs between SNR and acquisition times, as accelerated imaging will still result in data with sufficiently high SNR.

The $B_{1}^{+}$transmit profiles as generated by the CP mode at $9.4 \mathrm{~T}$ show highly inhomogeneous excitation across the sample, with several areas in the field of view being substantially under-flipped due to the transmit excitation pulses interfering destructively, resulting in very low transverse magnetization. These issues are rectified by using the kT-points parallel transmit method, which results in a much more homogeneous excitation across the region of interest. Crucially, a robust, homogeneous $B_{1}^{+}$excitation can help realize the SNR gain that can be afforded by the conformal, close-fitting receive coils. To further homogenise the overall $B_{1}^{+}$profile of the RF coil, future work could involve extending the number of transmit channels to 16 from the current 8 . These 16 transmit coils, albeit smaller in size from the existing design, can be tiled more effectively across the transmit former, with 8 channels on either side, thus providing superior and complete transmit coverage. In this case, on the transmit side decoupling is easier achieve using inductive decoupling methods. This will also enable finer control over pTx transmit modalities and thereby enable more effective and homogeneous excitation profiles. 


\section{Anatomical and quantitative imaging}

A combination of homogeneous Tx excitations and superior Rx signal SNR enabled us to acquire $100 \mu \mathrm{m}$ isotropic GRE $T_{2}^{*}$ weighted data over the entire post mortem human brain sample. It allows a clear definition and contrast of anatomical structures in the deep brain nuclei and especially in the cortical grey matter. For example, the stria of Gennari is clearly delineated in the coronal profiles shown in Fig 4.7.

The quantitative $T_{2}^{*}$ mapping, performed at $200 \mu \mathrm{m}$ isotropic, shows certain inherent advantages. Using multi-echo 3D GRE imaging for four different echo times we were able to generate a high quality $q T_{2}^{*}$ map using mono-exponential fitting. The resulting $q T_{2}^{*}$ volume provides superior quantitative contrast allowing us to effectively delineate white and gray matter areas, while the estimated $S_{0}$ map absorbs proton density and $T_{1}$ weighting, as well as transmit and receive field inhomogeneities, enacting bias field correction and providing a homogeneous contrast across the imaged volume. Technically, the measurement time for four 3D volumes at $200 \mu \mathrm{m}$ would be similar to that for one 3D volume at $100 \mu \mathrm{m}$, as the latter requires four times more phase encoding. However, in practice we could run the 4 echoes efficiently and with low gradient load as two double-echo acquisitions at slightly longer TR, increasing the time-efficiency somewhat. Importantly, the SNR in the $200 \mu \mathrm{m} q T_{2}^{*}$ data is also considerably higher. The $T_{2}^{*}$ values in the GM are higher than those in WM, with higher $T_{2}^{*}$ values for superficial GM layers. While the calculated $T_{2}^{*}$ values are lower than those reported for in-vivo studies, they are comparable with higher values that have been reported previously for post-mortem samples [127]. This evidently also depends on the treatment of the sample which includes post mortem interval (the time between death and fixation).

Consequently, the high resolution anatomical and quantitative data also have considerable potential towards the creation of 3D MRI-based 
anatomical atlases. As seen in Fig 4.6, the combination of superior resolution of the $100 \mu \mathrm{m} T_{2}^{*}$ weighted and the high SNR and homogeneous contrast from the $200 \mu \mathrm{m} q T_{2}^{*}$ estimated data enable us to label deep gray matter, allowing several subcortical structures to be distinguished on the basis of $T_{2}^{*}$ contrast. Furthermore, in Fig 4.7, the same datasets help in the characterisation of cortical layering in the coronal slice through the calcarine sulcus, highlighting the stria of Gennari in the primary visual area V1. Additionally, the $q T_{2}^{*}$ maps also help in further sub-layering as the pronounced $T_{2}^{*}$ contrast between the more myelinated infragranular layers and the less myelinated supragranular layers stands out particularly. 
Chapter 4. High resolution MRI neuroanatomy in intact human brains post mortem with a specialized 9.4T RF-coil 


\section{A modular, planar RF coil for high resolution, post-mortem imaging at $9.4 \mathrm{~T}$}

Contents

5.1 Introduction . . . . . . . . . . . . . . . . . . 121

5.2 Methods . . . . . . . . . . . . . . . . . . . . 122

5.3 Results . . . . . . . . . . . . . . . . . . . . . . . . 129

5.4 Discussion $\ldots \ldots \ldots \ldots \ldots$

Adapted from: Sengupta S., Fritz F. J., Hellenbrand R., Finger R., Wiggins C., and Roebroeck A. A modular RF coil platform for ex-vivo imaging of brain slices at 9.4T. In Proceedings of the 25th Annual Meeting of ISMRM, Honolulu, USA, page 2413, 2017 
Chapter 5. A modular, planar RF coil for high resolution, post-mortem imaging at $9.4 T$

\section{Abstract}

RF array coils designed for in-vivo imaging are not well suited to imaging ex-vivo brain samples. This holds especially true for imaging flat slabs or sections of brain tissue which would otherwise have a poor filling factor and/or relatively large distances between the coils and the sample. Previously, we showed the results of using a cylindrical phased-array receive ( $\mathrm{Rx}$ ) RF-coil to image large non-flat specimens $\left(\sim 80 \times 80 \times 80 \mathrm{~mm}^{3}\right)$ at very high resolutions using a large-bore human 9.4T MRI system [87]. Here, we present a modular 9.4T RF coil platform that can be used for imaging slabs of tissue as large as a coronal (diameter of $15 \mathrm{~cm}$ ), transverse or sagittal (diameter of $17 \mathrm{~cm}$ ) section of a brain hemisphere, with a thickness of 5-10 $\mathrm{mm}$. This imaged tissue can then be used for histological analysis after being being subjected to advanced tissue clearing techniques. A layered approach [148] was used while designing the receive array to ensure the coils' proximity to the sample under investigation. 


\subsection{Introduction}

Magnetic resonance imaging has found use in several in-vivo and exvivo studies due to its ability to provide tissue-specific contrast. These changes in tissue-based contrast however need to be validated using other imaging modalities in order to ensure that the changes in signal and contrast are due to pathology. This is usually achieved through histology, which remains the gold standard in determining tissue changes due to pathology. However, successfully coregistering data from histological sections with their MR images can be a challenging task, as the tissue under investigation will most likely suffer from deformations and shrinkage as a result of tissue fixation during histology [149, 150]. Additionally, the tissue sectioning required for histology, post MR imaging, would also have to be along the same orientations as the acquired MR data - a task which is not trivial and would be extremely difficult to achieve. Given that the slice thickness for MR imaging tends to be larger than that of a histological slice, the resulting coregistration can result in a mismatch between the respective datasets. One might also use software algorithms to stitch together several sequential histological slices, after correcting for tissue deformities, such that they match the slice thickness of the MR acquisition.

Thus, in order to perform optimised coregistration between ex-vivo tissue MRI and histology without errors, it would be ideal to perform MR imaging on the same slice used for histological analysis. As reported previously, bespoke RF coils have been constructed [151, 110] for imaging thin histological slices in both horizontal and vertical bore scanners. While these RF coil setups provide superior transmit and receive characteristics, they are inherently limited to extremely thin slices/samples and therefore require a long turnaround time when imaging and coregistering large, ex-vivo samples. Recent advances in histological methods have enabled the clearing and labelling of large, ex-vivo human brain samples, up to $5-10 \mathrm{~mm}$ in thickness [152]. Performing MR imaging on such sample sizes will require RF coils capable 
Chapter 5. A modular, planar RF coil for high resolution, post-mortem imaging at $9.4 \mathrm{~T}$

of producing sufficient Signal-to-Noise ratios (SNR) across the entire imaging volume for high mesoscale resolutions capable of distinguishing individual cortical layers and sub-layers.. It would also require the coil design to have a high filling factor, which can be achieved by ensuring that the coil design/layout conforms to the geometry of the sample [153]. Here we present a phased array coil setup with a layered, 23 channel receive ( $R x)$ section paired with a 7 channel transmit (Tx) coil. The flat receive array allows for a high filling factor and conforms to the dimensions and shape of the sample under investigation, while the transmit coil ensures the production of a uniform $B_{1}^{+}$field for high resolution MR imaging of large ex-vivo tissue slabs up to 16 $\mathrm{cm}$ in diameter and 5-10 $\mathrm{mm}$ in thickness.

\subsection{Methods}

\section{Electromagnetic simulation}

In order to determine the ideal transmit coil setup for the sample, we simulated 2 different transmit coil types and geometries, namely a 7 channel phased array and a double dipole setup. Both transmit coils were simulated in CST (Computer Simulation Technologies, 3DS, Darmstadt, Germany). The phased array Tx coil elements were first modelled as $35 \mu \mathrm{m}$ high perfect electrical conductor (PEC) traces on a FR4 board, using the software's 3D CAD designer. Each loop was designed to have 5 lumped element ports and a single discrete port. The 1 $\mathrm{mm}$ diameter wire used for connecting overlapping coil segments was also added to the simulation space. The double dipole design consisted of 2 dipoles of $20 \mathrm{~cm}$ length each, with a diameter of $1.2 \mathrm{~mm}$, spaced apart by $10 \mathrm{~cm}$, modelled as PECs. A $3 \mathrm{~mm}$ gap was inserted in the center of each dipole element to serve as a discrete port. While the lumped element ports accommodated the predefined fixed capacitors (RLC, series), the discrete ports were used for the tuning/matching 
capacitors and the $50 \Omega$ termination ports. All coil elements were modelled to accurately reflect the design on the coil, including the electrical properties of the sample and the RF shield.

The simulation setup was completed by modelling the sample as a 16 $\mathrm{cm}$ wide and $1 \mathrm{~cm}$ thick circular slab of brain/bio-tissue. Tissue parameters were defined using CST's in-built material library. A high resolution mesh was implemented ( $>2$ million mesh cells) in order to run the simulation accurately. It should be noted that the receive array, associated cable traps and coaxial cables were not added to the simulation space in order to avoid complex 3D meshing and also to save processing time. After completing the simulation, the in-built schematic tool was used to tune and match the coil elements to Larmor frequency at $50 \Omega$, followed by the calculation of $B_{1}^{+}$field characteristics using the software's MRI toolbox macro.

\section{RF coil construction}

The transmit and receive sections of the RF coil were constructed using FR4 and Rogers 4030 material respectively. 23 receive loops, each 4 $\mathrm{cm}$ in diameter, were laid out in a phased array configuration, with 19 loops accommodating a circular space, while 4 extra loops were placed at the corners to provide a total in-plane receive field of view of $14.5 \mathrm{x}$ $13.5 \mathrm{~cm}^{2}$. Each of the 23 receive coils were divided into 3 equal arc sections in order to allow for placement of capacitors and receive ports. Each of the 3 coil sections - spaced vertically apart by $0.2 \mathrm{~mm}$ - were placed on a separate layer in the $1 \mathrm{~mm}$ thick, 4-layer receive PCB. The loops were fashioned using $18 \mu \mathrm{m}$ high and $1 \mathrm{~mm}$ wide copper traces - with each loop being $4 \mathrm{~cm}$ in diameter- while maintaining the critical overlap of 0.20 times the coil diameter between all loops to ensure mutual decoupling. Given that the coil would be exclusively used for imaging flat sections of post-mortem brain tissue, it was imperative to design a completely flat phased array layout that would allow for an 
Chapter 5. A modular, planar RF coil for high resolution, post-mortem imaging at $9.4 \mathrm{~T}$

optimised filling factor while compensating for any perturbations in the receiver coil layout, and by extension the receive profile, if using wires when designing the loops. $3.5 \mathrm{~cm}$ high posts, 3D printed using ABS, were attached to the center of each coil element to thread the receive and detune lines emanating from each preamplifier.

A 7 channel phased array transmit coil was constructed on a $1 \mathrm{~mm}$ thick FR4 board using $35 \mu \mathrm{m}$ high and $2 \mathrm{~mm}$ wide copper traces, with each coil being $7 \mathrm{~cm}$ in diameter. The optimised overlap of 0.2 times the coil diameter was maintained here as well to minimise mutual inductance. A similar FR4 board was used to design an RF shield with 8 etched overlaps to reduce eddy current losses, and was placed $3 \mathrm{~cm}$ away from the transmit coil. A visual representation of both receive and transmit coils is shown in Fig 5.2.

Both transmit and receive coils were mounted on a semi-circular brace that would allow $90^{\circ}\left(-45^{\circ}\right.$ to $\left.+45^{\circ}\right)$ of rotation around the $z$-axis and $360^{\circ}$ of rotation around the y-axis, with the aim of performing quantitative susceptibility mapping on imaged tissue samples. The 3D design of the brace is shown in Fig 5.3

\section{Circuitry}

Each receive coil was connected to a circuit board containing the relevant circuitry, with the board placed perpendicular to the phased array PCB. Each loop is first connected to a lattice or LC balun circuit and a tuning and matching network comprising of a single fixed highvoltage matching capacitor and a high-voltage trimmer capacitor, for matching the coil's output impedance to $50 \Omega$ and for tuning the coil to resonance at 9.4T. First, a passive detuning circuity comprising of an LC circuit in parallel with a high-voltage diode was placed right after the balun circuit. Using the tuning and matching capacitors, the coil was first tuned and matched to $400 \mathrm{MHz}$ at $50 \Omega$ and then connected to 
a low-input impedance preamplifier using a trimmer capacitor. Additionally, the balun circuit incorporates an active detuning circuit across the matching capacitor. It is achieved by using a high-voltage PIN diode in series with the balun inductor, which along with the matching capacitor resonates at Larmor frequency. Therefore when the PIN diode is forward biased (during transmit), this parallel resonant circuit introduces a high impedance in series with the coil, effectively blocking current flow in the loop at the operating frequency.

While decoupling between adjacent neighbouring elements was achieved using critical overlap, decoupling between next-nearest elements was achieved by utilising preamplifer decoupling [21]. In order to transform the high impedance at the coil output to the corresponding low input impedance at the preamplifier input, a series matching trimmer capacitor was utilised in parallel with an inductor [77]. The parallel LC circuit resonates at the Larmor frequency, introducing the high series impedance in the loop, resulting in minimal current flow and minimises inductive coupling between next-nearest coil elements, notwithstanding any residual mutual inductance. The use of a trimmer capacitor for this purpose allows for finer adjustment of the impedance transformation, and by extension, the preamplifer decoupling between coil elements.

The PCBs containing the preamplifiers and detuning circuitry were arranged such that the preamplifiers themselves are aligned along the static $B_{0}$ field of the MRI scanner, thus minimizing any Hall effects that would otherwise affect the FETs in the preamplifiers [78]. Each preamplifier output was connected to its own cable trap - by making a solenoid from the coaxial cable connecting the preamp to the plug that connects to the magnet bed and bridging a variable capacitor across its ends and tuning it to a resonant frequency of $400 \mathrm{MHz}$. These traps help eliminate common mode currents and while suppressing interactions with the transmit coil.

The transmit coil consists of a 7 channel phased array, with 6 loops 
Chapter 5. A modular, planar RF coil for high resolution, post-mortem imaging at $9.4 \mathrm{~T}$

surrounding a central loop, each $7 \mathrm{~cm}$ in diameter. The loops were fabricated on FR4 boards utilising $35 \mu \mathrm{m}$ thick copper traces, while the width of each loop trace was $2 \mathrm{~mm}$. All loops was divided into 6 equal arc segments for accommodating capacitors and a Tx feed port, with three $5.1 \mathrm{pF}$ and two $7.5 \mathrm{pF}$ capacitors distributed along the length of the loop. Two high-voltage trimmer capacitor was placed in parallel at the feed port in order to tune and match the transmit loops to the resonant frequency of $400 \mathrm{MHz}$ at $50 \Omega$ noise match. The cables connecting the loop feed ports to the coil plug were routed along the center of the adjacent loops (virtual ground plane) in order to minimise their influence on the transmission and reflection parameters of the adjacent coil elements. High power PIN diodes were also soldered onto each coil in order to detune the transmit array during receive. An RF shield was also placed $2 \mathrm{~cm}$ away - along the y-axis - from the transmit coil. The RF shield was designed from a double sided FR4 board with 8 etched overlaps on either side - with the overlaps shifted by half their length on one side of the shield - in order to effectively minimise eddy current losses.
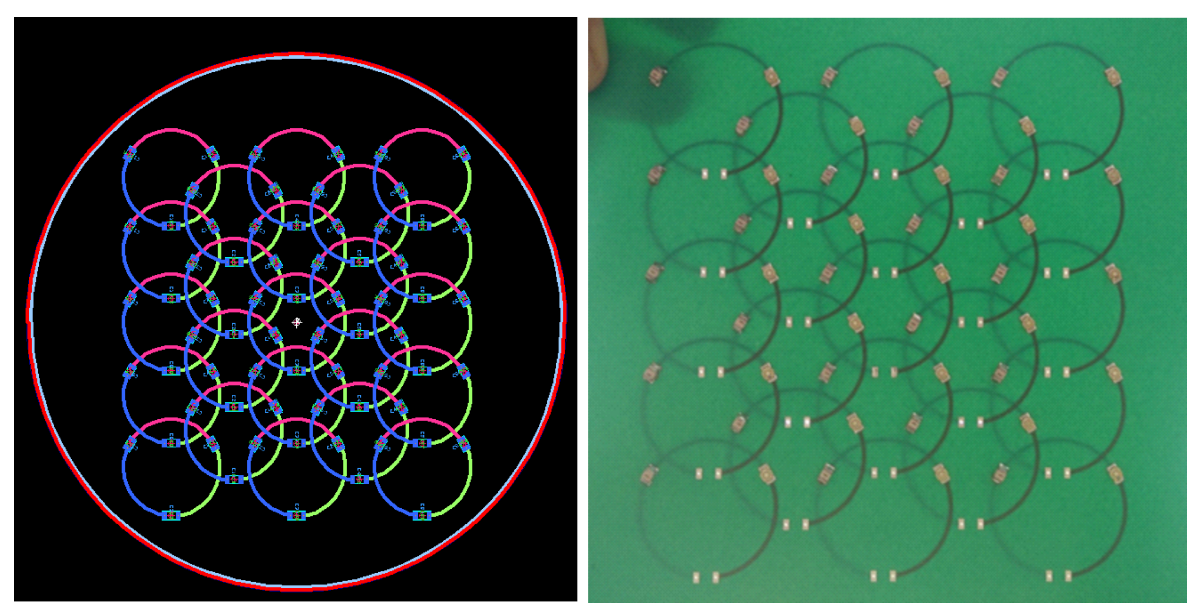

Figure 5.1: Receive array layout. (L) The 23 channel $R x$ coil layout as designed, with each coloured arc placed in a different PCB layer (R) The manufactured PCB layout populated with distributed fixed and tuning capacitors 


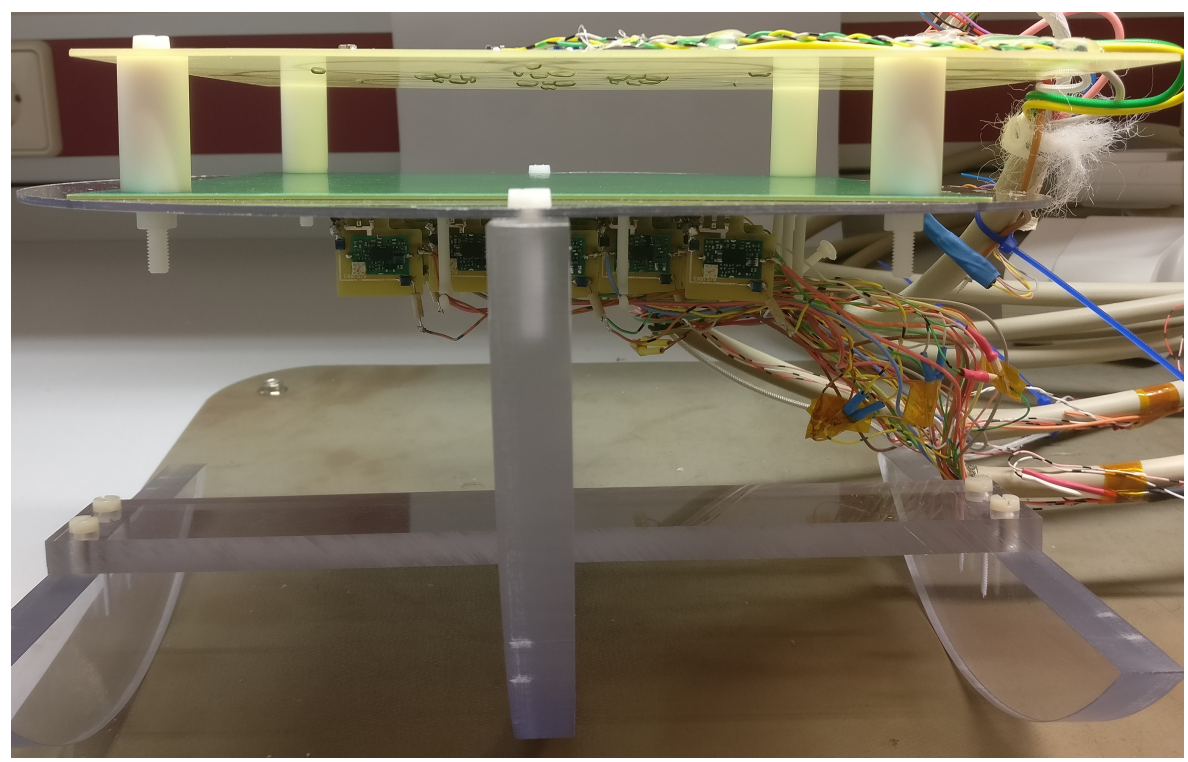

Figure 5.2: Planar coil layout. Side view of the 7 channel Tx coil (yellow) integrated with the 23 channel Rx coil (green) connected to low-noise, on coil preamplifiers, assembled on a static brace.

\section{Bench measurements}

In order to effectively quantify the electrical characteristics of the RF transmit and receive coils, bench tests were undertaken using an Agilent HP E5071C ENA series network analyser, a customised coil-plug bed incorporating both TIM and pTx plugs and a rectangular phantom $(49.8 \%$ demineralised water, $48.8 \%$ sucrose, $1.3 \% \mathrm{KCl}, 0.10 \%$ Dowicil; Max Planck Institute for Biological Cynernetics, Tubingen, Germany) as the load. Loaded and unloaded Q-ratios, individual receive coil decoupling and preamplifier decoupling for each element were measured. These measurements validated the coils' tuning and matching characteristics, preamplifier decoupling and active detuning.

Using distributed fixed and trimmer capacitors, each loop was tuned 
Chapter 5. A modular, planar RF coil for high resolution, post-mortem imaging at $9.4 \mathrm{~T}$

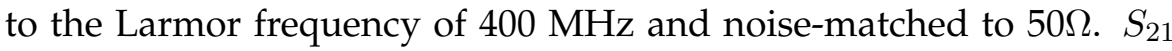
measurements were utilised to further measure coupling between neighbouring elements by directly connecting the coil outputs to two ports on the network analyser, while all other coil elements were kept in a detuned state. Preamplifier decoupling of a single loop was measured by noting the change in $S_{21}$ measurement using a pair of decoupled inductive probes: the coil was initially power matched to $50 \Omega$ while loaded with the rectangular phantom but without the preamplifier connected, and next the coil was terminated using the low input impedance preamplifier with all other surrounding coils detuned. The difference in the $S_{21}$ values between these two states provided the requisite data for preamplifier decoupling.

Similar bench tests were conducted for the 7 channel phased array transmit coil. Each transmit loop was carefully tuned and matched to $400 \mathrm{MHz}$ and $50 \Omega$ respectively, with the loops directly connected to the network analyser and loaded with the rectangular phantom. The coupling between adjacent elements was measured as the $S_{21}$ parameters across coil outputs. Using decoupled inductive probes, the active detuning for the transmit loops was recorded as $S_{21}$ measurements by forward biasing the diode on the coil under investigation, while keeping the other loops in the array in a detuned state.

\section{MRI Data Acquisition and Reconstruction}

Data acquisitions were performed on a $830 \mathrm{~mm}$ human size bore research 9.4T Siemens MAGNETOM MR system (Siemens Healthcare, Erlangen, Germany). $B_{0}$ and $B_{1}^{+}$shimming were performed before acquiring high resolution anatomical data using a $7 \mathrm{~cm}$ wide, $1 \mathrm{~cm}$ thick slab of brain tissue, procured as a coronal slice of a human occipital lobe . A localizer was initially acquired for spatial-localization reference; then fieldmap [117] and DREAM [118] protocols were acquired to characterise $B_{0}$ and $B_{1}^{+}$map profiles, respectively, across the 
sample. MATLAB (MathWorks, MA, USA) routines were used to optimise $B_{0}$ and $B_{1}^{+}$shimming using those acquisitions [119, 120]. The fieldmap sequences acquired were shimmed iteratively to improve $B_{0}$ homogeneity.

Acquisitions were performed with a custom 3D gradient echo (GRE) pulse sequence, modified to allow large matrix sizes and to use a composite parallel excitation pulse using the kT-points technique for $B_{1}^{+}$ homogenization [121]. The 3D GRE sequence was used to acquire high resolution isotropic $T_{2}^{*}$ weighted data at: $250 \mu \mathrm{m}$ (Repetition time $(\mathrm{TR}) /$ Echo time $(\mathrm{TE})=23 \mathrm{~ms} / 6.21 \mathrm{~ms}$, flip angle $(\mathrm{FA})=20^{\circ}$, readout bandwidth $(\mathrm{BW})=120 \mathrm{~Hz} / \mathrm{px}$. A $0 \mathrm{~V}$ transmit power noise reference scan was acquired to calculate a noise covariance matrix to be used for optimal coil-combination in image reconstruction.

\subsection{Results}

The calculated coil Q-ratio $\left(\mathrm{Q}_{U} / \mathrm{Q}_{L}\right)$ for a single isolated element (with all other loop depopulated) was 3.2, while the Q-ratio of a single receive element surrounded by 5 detuned loops was 2.8. All receive loops were matched to $-22 \mathrm{~dB}$ or better, when loaded with an ex-vivo brain sample. The active PIN diode detuning afforded an additional $26 \mathrm{~dB}$ of isolation between the tuned and detuned states, while preamplifier decoupling allowed for $20 \mathrm{~dB}$ to $24 \mathrm{~dB}$ of isolation. Decoupling between adjacent receive coil elements was $-17 \mathrm{~dB}$ or better, while the decoupling between the next nearest elements was $-20 \mathrm{~dB}$ or better. The high decoupling between elements is also due to the flat geometry of the layout which spaces next-nearest coil elements further apart. The noise correlation matrix for the 23 channel receive coil, as shown in Fig 5.4 , obtained through ex-vivo scanning, shows a maximum correlation of $38 \%$ and an average of $12 \%$.

The comparison between the two different transmit coil simulations 
Chapter 5. A modular, planar RF coil for high resolution, post-mortem imaging at $9.4 T$

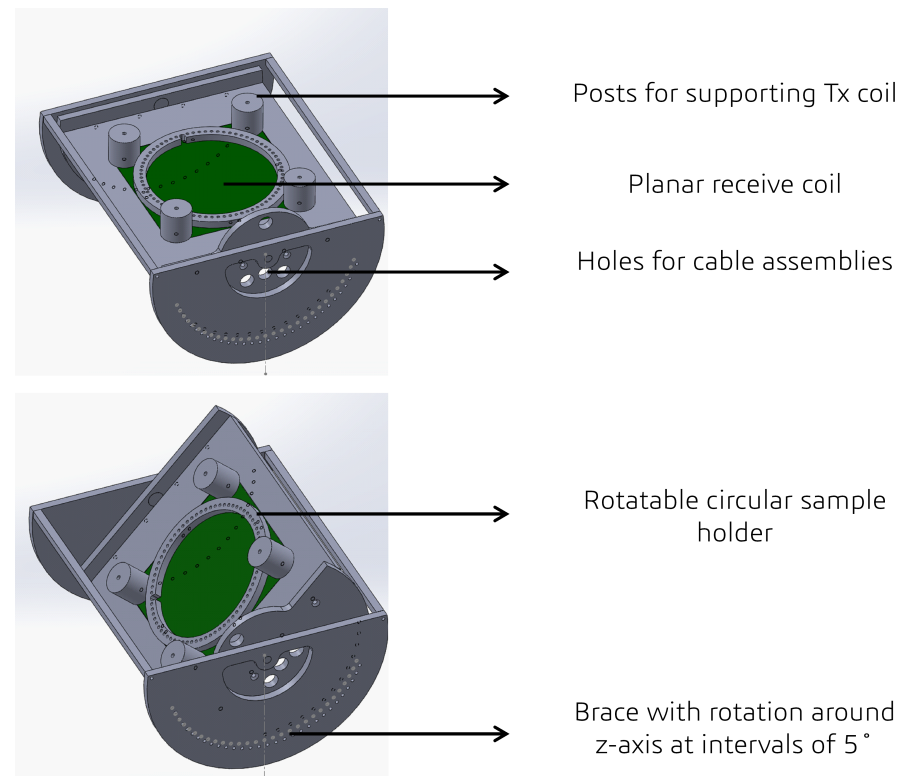

Figure 5.3: 3D representation of rotatable brace for the planar coil

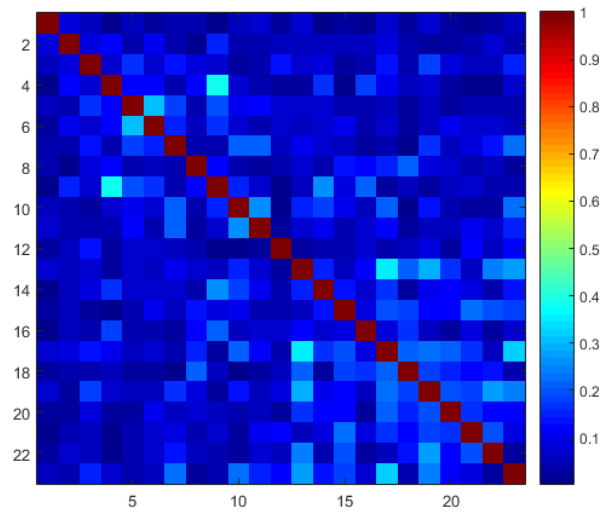

Figure 5.4: Noise correlation matrix. The noise correlation matrix for the 23 channel receive array

is shown in Fig 5.5. The simulation results show that the 7 channel phased array layout provides a more homogeneous $B_{1}^{+}$field across 

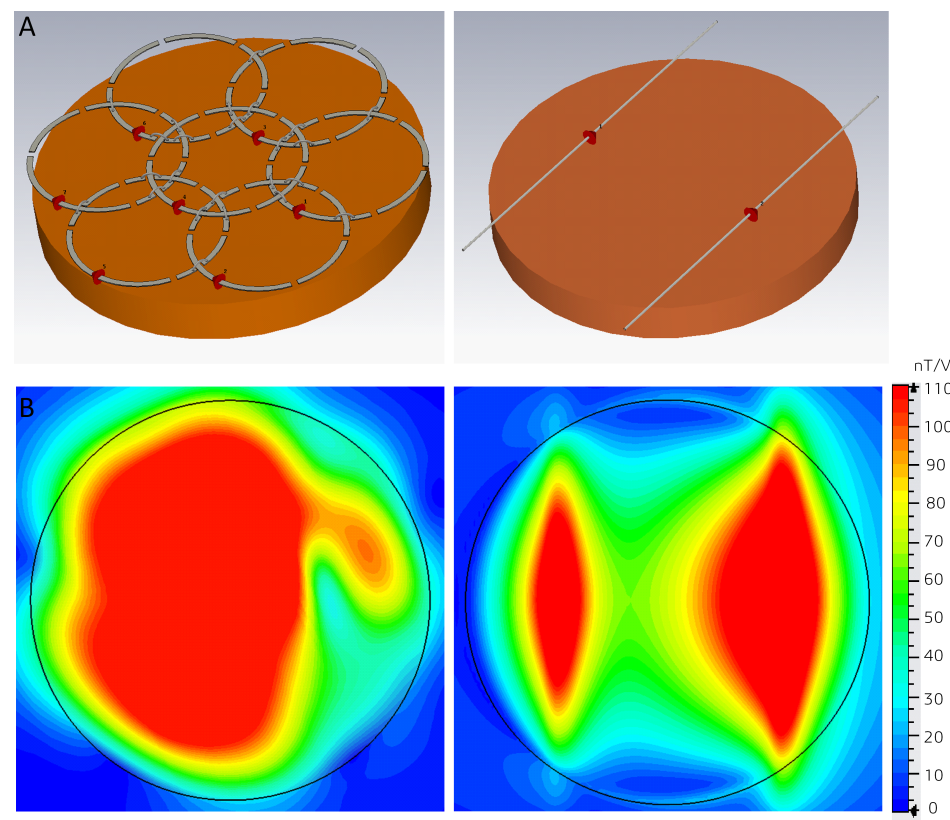

Figure 5.5: EM simulation setup and $B_{1}^{+}$maps. (A) The 7 channel phased array transmit coil and the double dipole transmit EM simulation setup. The ground planes are hidden to provide a better view of the coils (B) The resulting simulated $B_{1}^{+}$field maps for both Tx layouts, with the black circle representing the sample space

the flat sample than the double dipole setup. The phased array layout will also allow for more flexibility when using the kT-points method for $B_{1}^{+}$homogenisation due to the larger number of channels, leading to a more effective $B_{1}^{+}$shim.

Fig 5.6 shows the $250 \mu \mathrm{m}$ isotropic proton-density weighted GRE acquisition, with sufficient SNR to delineate white and gray matter areas, as indicated by the yellow arrows in the figure. The SNR map indicates a relatively homogeneous $B_{1}^{-}$field profile for the receive coil, while the central area of the sample shows decreased SNR performance due to sub-optimal $B_{1}^{+}$transmit field excitation in that region. 
Chapter 5. A modular, planar RF coil for high resolution, post-mortem imaging at $9.4 T$
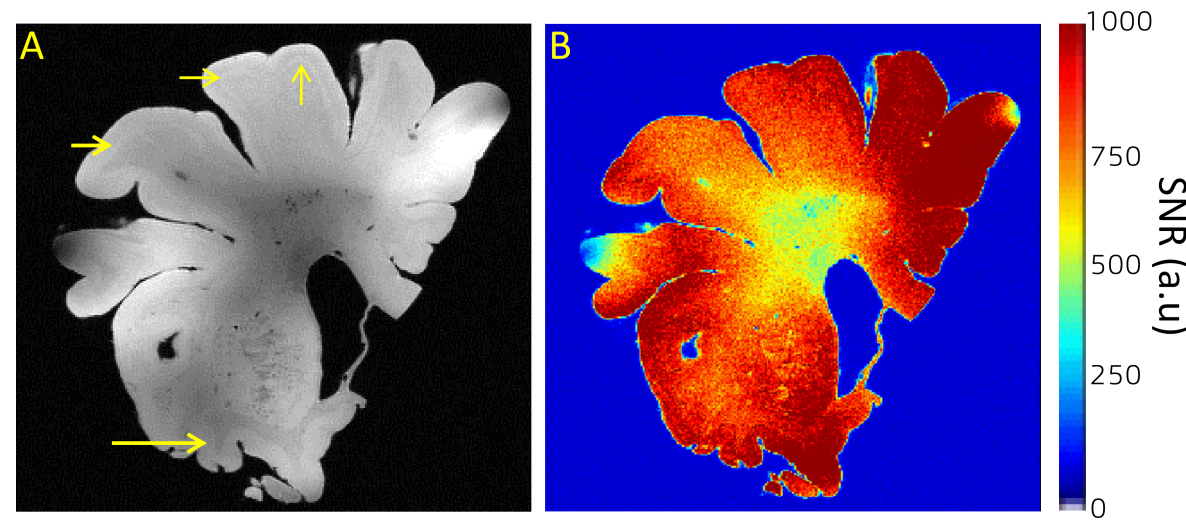

Figure 5.6: (A) Anatomical image from a GRE scan at $250 \mu \mathrm{m}$ isotropic (B) Signal to noise ratio for the $8 \mathrm{~cm}$ wide ex-vivo sample across the same slice

\subsection{Discussion}

Most MR imaging of histological tissue so far has been undertaken with small diameter RF coils used in narrow-bore, pre-clinical animal scanners or vertical bore scanners. Post-scan registration of histological data with MRI images has been an issue as the tissue under investigation will be subject to deformities and shrinkage as a result of tissue fixation. This has been addressed by using a stained histological slice for MR imaging and then consequently using the same slice for microscopy, thereby reducing potential coregistration errors. However, with the emergence of new staining methods for thick, ex-vivo samples (up to 5-10 mm thick) [152] and light-sheet microscopy techniques, MR imaging and subsequent histological analysis can be performed on the same slab of ex-vivo human tissue, potentially allowing for fewer errors during image coregistration.

We constructed a working prototype of a rotatable 23 channel receive / 7 channel transmit phased array planar coil, with the aim of eventually performing high resolution anatomical and quantitative imaging on large, flat human brain samples. Two different transmit coil types 
- namely a multi-channel phased array and a double-dipole setup were investigated using EM simulation for determining the optimal transmit coil geometry suitable for this imaging setup. The 7 channel phased array layout provides a relatively homogeneous $B_{1}^{+}$excitation profile across the sample FoV, while also allowing for the implementation of parallel transmit (pTx) using kT-points method in order to optimise said $B_{1}^{+}$field pattern. A layered approach was used for implementing the receiver coils, as each $\mathrm{Rx}$ coil was segmented into three individual arcs, with each arc placed on a different layer of a 4-layer PCB. This allows for a completely flat resting surface for the sample and also helps realise the optimised coil overlap for mutual decoupling of adjacent coil elements. This flat coil geometry also renders the sample close to the surface of the coil, increasing coil sensitivity and enhancing the coil filling factor.

Both transmit and receive coils' performance was validated through a combination of bench tests and ex-vivo imaging. All transmit and receive coils were sufficiently decoupled from each other, while active detuning and preamplifier decoupling methods were implemented on the Rx side, providing further isolation between adjacent Rx coil elements, evidenced by the low noise correlation exhibited by the receive channels.

The SNR achieved shows a homogeneous distribution across the sample, with a pocket of low SNR in the center of the sample due to inefficient transmit field in that area. This particular issue can be rectified with the use of optimised kT-points excitation pulses. While the proton density weighted $250 \mu \mathrm{m}$ isotropic GRE of the sample shows clear delineation of white and gray matter, the available contrast and resolution can be further increased by optimising the sequence parameters to match the sample. Additionally, there is the unique possibility of rotating the sample container on top of the coil surface and tilting the coil surface, which provides 2 full degrees of rotational freedom, to position the sample with respect to the main magnetic field $B_{0}$ and the gradient system axes. This enables advanced phase contrast and 
Chapter 5. A modular, planar RF coil for high resolution, post-mortem imaging at $9.4 T$

quantitative susceptibility imaging, having arbitrary orientation of the magnetic field vector with respect to the sample. Along the same lines, it would allow diffusion imaging with up to square-root of 3 more diffusion gradient amplitude and hence up to 3 times higher b-values, by rotating the sample and using the full vector-summed amplitude of 3 simultaneous gradients. 


\section{Summary and Discussion}

Contents

6.1 Summary . . . . . . . . . . . . . . . . . 137

6.2 Discussion . . . . . . . . . . . . . . . . 140 



\subsection{Summary}

In Chapter 2, we show the benefits of constructing a semi-cylindrical, shape optimised, 16ch phased array Rx / 4ch phased array Tx RF coil targeted at acquiring high resolution, in-vivo anatomical and functional MRI data of the human visual cortex at 7T. The close fitting coil former allows both transmit and receive coils to lie in close proximity to our region of interest - namely the primary and secondary visual areas and does not require tuning and matching for individual subjects, resulting in reduced scan preparation times. The RF coil's transmit field characteristics were simulated using EM simulation software and validated through acquired flip angle maps, while SAR analysis was also undertaken to determine safety limits for human use in accordance with IEC 60601-2-33 standards. The coil was also subjected to thorough bench tests, using a human torso shaped phantom filled with tissue-mimicking dielectric solution. The coil's performance was assessed through several MRI acquisitions, whereby its SNR, receiver noise correlation, $\mathrm{tSNR}$, and g-factors were calculated and compared to that of a commercial 32ch Rx / 1 ch Tx coil. fMRI studies at standard and sub-millimeter resolutions was acquired across both coils on human subjects, using a standard visual stimulus. The constructed transmit and receive sections showed good interelement decoupling and low mean noise correlation values. The SNR for the $16 \mathrm{ch}$ coil was about 1.5 times that of the standard coil in the human occipital lobe, and exhibited lower g-factors for accelerated imaging. The frontallyopen design of the RF coil presented the human subjects with a viewing angle of almost $30^{\circ}-3$ times that of the commercial coil. This was reflected in the fMRI data acquired, as the combination of a wide viewing angle and dense receiver coil layout in the region of interest results in the 16 channel Visual Arc coil showing activation deeper into the occipital lobe, both in the superior-inferior and anterior-posterior directions at $1.2 \mathrm{~mm}$ and $0.8 \mathrm{~mm}$ isotropic resolutions. 
Chapter 3 describes the contruction, testing and validation of a 16 channel receive coil for high resolution anatomical and quantitative imaging of large, ex-vivo brain samples using a wide-bore 9.4T scanner, with the coil's performance tested through bench tests and ex-vivo scanning of a human occipital lobe. The cylindrical, phased array layout of the receive channels, with an imaging volume of $80^{3} \mathrm{~mm}^{3}$, covers the entire sample, while ensuring that the small receive coils are in close proximity with the sample. The resulting noise correlation was an average of $22 \%$, with each coil showing substantial $B_{1}^{-}$penetration depth. Compared to a 31ch $\mathrm{Rx} / 16$ ch transmit head coil at $9.4 \mathrm{~T}$, the $16 \mathrm{ch}$ Rx large sample post-mortem coil shows a five-fold increase in SNR in the periphery, and a two-fold increase in the center. The transmit profiles generated by a CP mode excitation using a dedicated 16 ch Tx coil for 9.4T show the expected high central fields dropping off towards the periphery, since the sample dimensions are close to the wavelength at 9.4T. Additionally, further $B_{1}^{+}$inhomogeneity was detected in the anterior-porterior direction. Using a kT-points excitation method, a more homegeneous excitation profile was achieved. The differences between the $B_{1}$ corrected and uncorrected SNR maps show the importance of having additional control over $B_{1}$ through kT-points transmit methods. The high SNR inherent to the coil allowed us to acquire $100 \mu \mathrm{m}$ and $60 \mu \mathrm{m}$ isotropic $T_{2}^{*}$ weighted data, while a multi-echo 3D GRE sequence was used to acquire multi-contrast $T_{2}^{*}$ weighted data at $200 \mu \mathrm{m}$ for quantitative $T_{2}^{*}$ mapping of the entire human occipital lobe. The $q T_{2}^{*}$ mapping at $200 \mu \mathrm{m}$ isotropic shows a clear definition of cortical architecture details, specially in the Stria of Gennari, with the estimated $T_{2}^{*}$ values being comparable to with previously reported values in post-mortem samples.

Chapter 4 expands upon the concepts outlined in Chapter 2 in order to build and characterise an RF coil with a conformal phased array receiver geometry and a multi-channel transmit section, with the aim of achieving high resolution anatomical and quantitative MR imaging of intact, post-mortem human brains at 9.4T. A 3D conformal sample container for post-mortem human brains was first designed in SolidWorks 
using anatomical MRI data, then surface-rendered and extruded in all directions and designed into a volumetric container in 2 halves. The receiver former was then modelled as a precise fit around the sample container and 24 receive coils were laid out in a phased array configuration on the former. The 8 transmit loops were laid out on two halves of a hollow, ellipsoidal shell, with 4 on each side. The coil's performance was evaluated through bench tests and ex-vivo scanning, and its SNR compared to that of a in-vivo, whole head coil at 7T. The measured SNR showed a five-fold increase in SNR at the periphery and a three-fold increase in the center for the whole brain coil over the whole head coil at 7T. The whole brain coil also showed a low, average noise correlation of $20 \%$, while certain coil pairs exhibited a high noise correlation due to a combination of sub-optimal overlap - and thus reduced decoupling - and shared acquired resistance through the sample under investigation. The 24 receive loop design also allowed for accelerated imaging in a single phase encode direction up to a factor of 3 , showing highly independent spatial selectivity of the loops and low g-factors. This, coupled with the high SNR capability of the coil can allow for significant trade-offs between SNR and acquisition times. The inhomogeneous $B_{1}^{+}$profile generated by a CP mode transmit excitation was successfully rectified by using a kT-points parallel transmit method, resulting in a homogeneous excitation in the region of interest. High resolution anatomical and quantitative MR data was acquied at $100 \mu \mathrm{m}$ and $200 \mu \mathrm{m}$ respectively. The $100 \mu \mathrm{m}$ isotropic GRE datasets enabled clear definition and contrast of anatomical structures, especially in cortical gray matter, with the Stria of Gennari in the primary visual cortex clearly visualised. High quality $q T_{2}^{*}$ maps were generated through mono-exponential fitting of multi-echo, $200 \mu \mathrm{m}$ isotropic $T_{2}^{*}$-weighted data, with the resulting $q T_{2}^{*}$ volumes showing superior quantitative contrast, effectively helping delineation of cortical layering. These high resolution anatomical and quantitative data have the potential of being used towards the creation of 3D MRI-based anatomical atlases. This combination of superior resolution of the $100 \mu \mathrm{m} T_{2}^{*}$ weighted data and the high SNR and homogeneous contrast from the $200 \mu \mathrm{m} q T_{2}^{*}$ estimated data can enable us to label deep gray matter and 
help distinguish several subcortical structures on the basis of $T_{2}^{*}$ contrast.

In Chapter 5 we describe the development and construction of a layered, 23 channel receive / 7 channel transmit planar coil for high resolution histological MR imaging of large, ex-vivo tissue slabs of thickness of up to $1 \mathrm{~cm}$, at $9.4 \mathrm{~T}$. Electromagnetic simulations were undertaken to determine the ideal transmit coil geometry, capable of producing a homogeneous $B_{1}^{+}$field in the FoV. On the receive side, $4 \mathrm{~cm}$ wide circular loops were segmented in three equal arcs and each placed in a different layer of a 4-layered PCB, providing a flat surface for sample placement and also increasing the coil filling factor. All transmit and receive coils were sufficiently decoupled from each other using a combination of mutual and preamplifier decoupling methods, with the receive channels exhibiting very low noise correlations due to the flat geometry. The SNR exhibited by the coil shows a mostly homogeneous $B_{1}^{-}$distribution, which can be suitably improved by utilising more aggressive parallel transmit shimming methods. The anatomical data acquired using a coronal slice of a human occipital lobe at $250 \mu \mathrm{m}$ isotropic shows delineation between white matter and gray matter areas, and can be further improved upon by using sequence parameters optimised to the sample under investigation.

\subsection{Discussion}

The aim of this thesis was to investigate and develop RF coils for high resolution MR imaging, with an emphasis on anatomical and functional imaging of the human cortex, at ultra-high fields of 7T and 9.4T. The studies presented within this dissertation demonstrate the importance of developing sample and target region - specific RF coils towards achieving high resolution MR imaging of both in-vivo subjects and ex-vivo samples. With the introduction of higher field strength MRI scanners, particularly ultra-high fields of $7 \mathrm{~T}$ and above, and the 
availability of bespoke RF coils aimed at high resolution imaging, perspectives arise on the imaging of cortical layers. While the design and implementation of bespoke RF coils remains important, special care must be taken in ensuring the compatibility and performance of associated coil hardware, namely preamplifiers, power splitters and cable traps. The electromagnetic simulations undertaken also provide a specific starting point and reference for coil design and development, and a close conformity between simulations and measurement results is highly desired when evaluating coil performance and subject safety.

In the chapters of this thesis, RF transmit and receive coils are developed, simulated and electrically characterized through numerical FDTD simulations, bench tests and MRI measurements. Further characterisation of parallel imaging performance was performed, along with SAR simulations aimed at implementing patient safety for invivo acquisitions. For all developed coils, quantitative and qualitative comparisons (SNR, $B_{1}^{+}$and $B_{1}^{-}$, g-factors, image quality, layer differentiation through contrast) with standardised or commercial RF coils were undertaken to show the advantages of used sample-specific RF coils for high resolution imaging. To this effect, it is important for researchers to have access to specific imaging hardware (RF coils) and sequences that are optimised for undertaking MR acquisitions of localised brain areas, both for in-vivo subjects and ex-vivo samples. When developing RF coils, several factors must be kept in mind - specifically the spatial resolution to be achieved, transmit and receive field homogeneity and their subsequent penetration into the sample, and subject safety through SAR assessments where applicable. While ultra-high field imaging (7T) has become an established research modality with clinical integration on the horizon, imaging at higher field strengths $(\geq 9.4 \mathrm{~T})$ is not without its challenges. At field strengths of 7T and above, the RF wavelength becomes similar to the dimensions of the human body, leading to $B_{1}$ field inhomogeneities and increased or sub-optimal SAR. 
Commercial RF coils

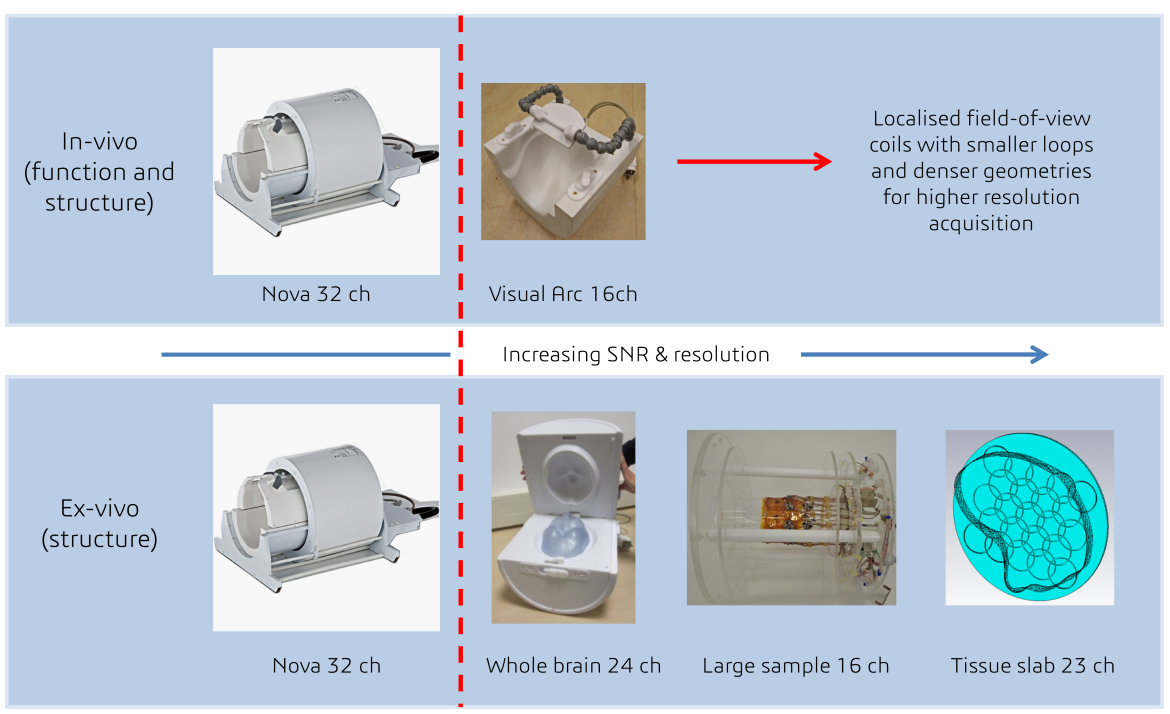

Farthest loop

distance from

sample center
Purpose-built RF coils

and denser geometries

higher resolutio acquisition

$2 \mathrm{~cm}$

Figure 6.1: Summary of RF coil designs presented in this thesis and an illustration of how they advance beyond the general-purpose, state-of-the-art commercial RF coils, for both in-vivo imaging (top) and ex-vivo imaging (bottom).

Fig 6.1 gives an overview of the development and implementation of specialized RF coils in this thesis and how this is critical in advancing high resolution small FoV imaging beyond general-purpose RF coils. For both in-vivo and ex-vivo imaging, the most essential gains achieved come from placing smaller diameter coils closer to the sample, as illustrated at the bottom of the figure. In ex-vivo sample imaging, there is greater possibility for this, especially when imaging samples smaller than the intact human brain, which is the case for both the large sample and tissue-slab coil.

With respect to in vivo imaging, an obvious further advance would be 
an even denser phased array of smaller loop coils covering the visual cortex ROI. In-house prototypes comprising of 24 channel, $5 \mathrm{~cm}$ loops at $7 \mathrm{~T}$ and 32 channel, $4 \mathrm{~cm}$ loops at $9.4 \mathrm{~T}$ are currently being tested. These diameters, at these respective field strengths, are likely close to the optimal SNR configuration for cortical imaging, i.e for imaging to a depth of up to $3 \mathrm{~cm}$ from the skull [154, 155]. Nevertheless, success with high resolution cortical fMRI at 7T has been achieved with even smaller loop sizes of 1-2 cm [71]. This suggests that the smallest loop size for high resolution cortical imaging at UHF is an issue that still deserves investigation. A recent study - undertaken with the invivo RF coil presented in chapter 2 - discusses the acquisition of fMRI data with the aim of achieving true in-plane laminar spatial resolution, negating the need for upsampling and averaging over an extended region of interest. A novel, layer-specific acquisition strategy that can sample cortical depths at resolutions of up to $100 \mu \mathrm{m}$ using anisotropic voxels and FLASH is showcased, and compared to data acquired using conventional isotropic voxel EPI acquisitions (Fig 6.2) [156]. It can be seen that the sharp increase of BOLD signal towards the pial surface, from large draining veins, is greatly ameliorated (Fig 6.2 C) with a very high resolution, in-plane acquisition strategy. As signal from the large draining veins is a nuisance in analysing layer-specific neural activity related signal, this shows the potential of purpose-built, UHF RF coils. With respect to the transmit side for in-vivo imaging, the implementation of full parallel transmit techniques, in contrast to the static RF shimming techniques used in chapter 2 , would help in homogenising the $B_{1}^{+}$fields even further in the visual cortex [120]. However the control on SAR would have to be carefully implemented and monitored through EM simulations.

A different type of transmit coil design that has found increasing use in high-field MRI is the electric dipole. With a sufficient number of dipole elements encircling a sample or subject ROI, dipole antennas can not only achieve higher central $B_{1}^{+}$and $B_{1}^{-}$fields - and thus SNR - but can also provide extended longitudinal coverage along the magnetic Z-axis, effectively covering brain areas such as the cerebellum and 

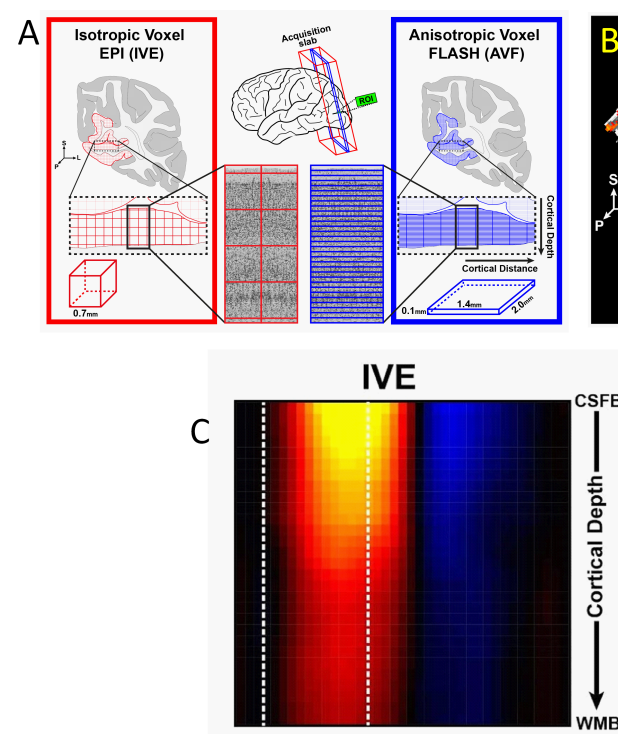

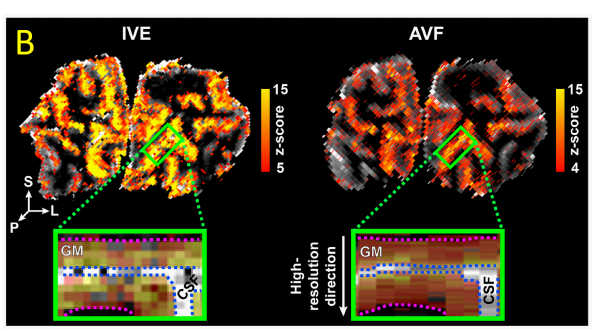

AVF

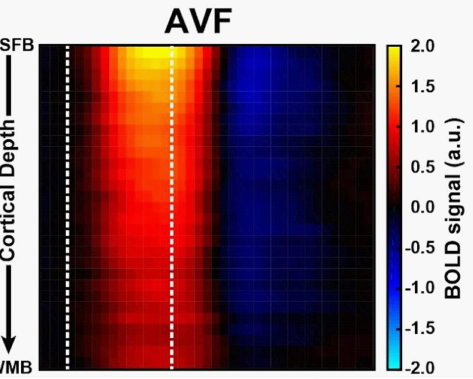

Figure 6.2: (A) Illustration of the IVE (left, red) and the novel AVF (right, blue) acquisition schemes for layer-specific fMRI, highlighting the differences in the sampling of cortical depths between the two approaches (B) Example of single-subject, single-run activation maps for the flickering checkerboard stimulus, for the IVE (left) and AVF (right) acquisitions. The green rectangle indicates the ROI in a coronal slice through the occipital lobe. In the zoomedin panels, the dotted pink line indicates the GM-WM border and the blue line the GM-CSF border. In the activation maps, the values for CSF are masked out to better visualize the 'flat' cortical patch of interest (C) Bipolar color-map representation of the average hemodynamic timecourses for thirty cortical depths from the IVE (left) and the AVF (right) acquisitions. The white dotted lines on the color-map in Figure 3a indicate the stimulus-on period. Reprinted with permission from Kashyap S.S., et al [156]

brain stem [82, 157]. While dipole arrays have been used for human head imaging, they find even more extensive applications in human body imaging i.e spinal [158] and prostate imaging [159], to name a few. This is primarily due to their ability of generating higher transmit and receive efficiency from a given depth onwards, rendering them preferable over surface arrays when it comes to imaging ROIs located 
deep inside the human body [22]. This suggests that their future benefits for whole body, notably chest and abdominal imaging, are likely greater than for imaging the human head.

With respect to ex-vivo imaging, this thesis presents a range of coils for human, post-mortem sample imaging. This range extends from the imaging of intact human cadaver brains (Chapter 4) to large human brain samples such as the human occipital lobe (Chapter 3), to 5-10 mm thick and potentially very wide human brain tissue slabs (Chapter 5). A general theme over post-mortem RF coil building is to extend the field-of-view from earlier post-mortem human tissue investigations in small bore, translational MR systems while retaining the very high resolution which enables the delineation of cortical layers. Where the tissue slab coil and large sample 16 channel coil have very controlled geometries and equally sized loops, the whole brain coil with its convoluted, conformal receive geometry has differently sized and variably overlapping loops necessitated by the geometry. Extending the number of receive channels on the whole brain coil would require smaller loops with very good decoupling, which is more difficult to achieve on a non-convex former. On the transmit side however, the number of channels could potentially be increased to 16 in order to provide even better control over $B_{1}^{+}$using pTx techniques. For the large sample coil, adding another row of 8 receive coils would extend the coverage along the cylindrical axis for longer samples. However, interesting human brain samples (such as the human occipital and temporal lobes) are largely covered by the $8 \mathrm{~cm}$ field-of-view along the cylindrical axis. As argued in Chapter 3 , the oversized transmit coil used for the study is sufficient for the purpose even though it is quite power-inefficient. Nevertheless a more closely fitting, smaller diameter transmit coil might afford small gains in power efficiency and $B_{1}^{+}$ homogeneity achieved through RF shimming or pTx methods. For the planar coil described in Chapter 5, we show the design, simulation and first implementation of the prototype, including high resolution GRE imaging. The ultimate aim is to achieve resolutions for thick tissue slabs higher than that possible with the large sample 16 channel coil. 
In addition, there is the unique possibility of rotating the sample container on top of the coil surface and tilting the coil surface, which provides 2 full degrees of rotational freedom, to position the sample with respect to the main magnetic field $B_{0}$ and the gradient system axes. This enables advanced phase contrast and quantitative susceptibility imaging, having arbitrary orientation of the magnetic field vector with respect to the sample. Along the same lines, it would allow diffusion imaging with up to square-root of 3 more diffusion gradient amplitude and hence up to 3 times higher b-values, by rotating the sample and using the full vector-summed amplitude of 3 simultaneous gradients.

This ex-vivo imaging capability opens the door to layer-specific, myelin-dominated imaging of large parts or even the entire human neocortex. As illustrated in Chapter 4, ultra-high resolution whole brain imaging could provide access to the myeloarchitecture of the human brain throughout all of its areas, with the resolution of single layers. Of course, this MRI contrast requires validation through histological methods, for the biophysical and molecular underpinnings of MRI contrast such as $T_{2}^{*}$. A challenge here is provided by the fact that the work in this thesis allows very large, 3D field-of-view imaging whereas histology is nowadays still performed on 2D sections thinner than a $100 \mu \mathrm{m}$. However, state-of-the-art histology techniques can provide an opening, as shown in Fig 6.3. Here it is shown that it is now possible to perform optical clearing - i.e. making transparent to light - of very large, up to $5 \mathrm{~mm}$ thick, human post-mortem brain samples, for fluorescent light microscopy imaging. This enables classification of cytoarchitectonic layers over the full cortical depth, and characterizing cortical architecture in 3D from the scale of cortical areas, easily colocalizable to MRI scans (Fig 6.3a), to that of single cells (Fig 6.3f). Histologically validated layer-specific MRI contrasts post-mortem could play an important role in defining possibilities for in-vivo imaging, of both layer-specific function and structure. 


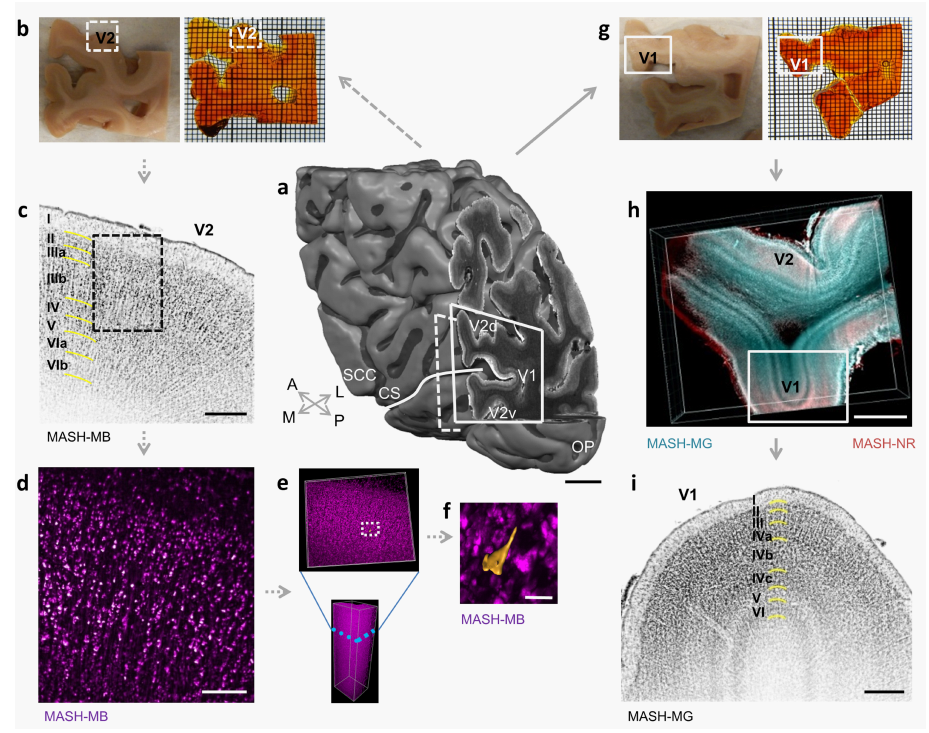

Figure 6.3: Optical tissue clearing allows characterisation of human cortical cytoarchitecture in large formalin fixed samples over a large range of scales. (a) 3D MRI reconstruction of the human occipital lobe sample with primary and secondary visual cortex around the calcarine sulcus (see chapter 2 of this thesis). V1: primary visual cortex; V2: secondary visual cortex; V2v: ventral V2; V2d: dorsal V2; CS: Calcarine Sulcus (white line); SCC: Splenium of the Corpus Callosum; OP: Occipital Pole; A: anterior; P: posterior; M: medial; L: lateral. (b) The anterior $5 \mathrm{~mm}$ thick sample (dashed line in a), before (left) and after staining and clearing (right). (c) LSFM imaging of the MASH-MB stain in V2 (inverted greyscalemap) in the dashed box in b, with cytoarchitectonic layering characterisation (left). (d) Higher magnification LSFM of the dashed box in c. (e) the entire 3D LSFM stack at the position of $d$ with magnification of a sub-stack at the blue dashed line (f) a 3D surface reconstruction of a pyramidal neuron cell body from LSFM data at the dashed box in e, imaging depth $1066 \mu \mathrm{m}$. (g) The posterior $5 \mathrm{~mm}$ thick sample (solid line in a), before (left) and after staining and clearing (right). (h) LSFM imaging of dual MASH-NR soma staining and MASH-MG nucleus staining in V1 and V2 in the dashed box in g. (i) higher magnification LSFM imaging of the MASH-MG channel in the solid box in $\mathrm{h}$ (inverted orientation), with cytoarchitectonic characterisation of V1 cortical layering (middle). LSFM: Light sheet fluorescence microscopy. MASH-NR: Fluorescent red spectrum cell body label. MASH-MB: Fluorescent far-red spectrum cell body label. MASH-MG: Fluorescent far-red spectrum nuclear label. Thin grid (b,g): $1 \mathrm{~mm}$. Scalebars: a: $10 \mathrm{~mm}$; h: $2 \mathrm{~mm}$; c,i: $500 \mu \mathrm{m}$; d: $200 \mu \mathrm{m}$; f: $50 \mu \mathrm{m}$. Reprinted from [152] under a CC-BY-NC-ND 4.0 International license. 



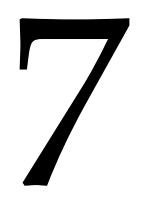

Valorisation 



\section{Knowledge Valorisation}

Knowledge valorisation refers to "the process of creating value from knowledge, by making knowledge suitable and/or available for social (and/or economic) use and by making knowledge suitable for translation into competitive products, services, processes and new commercial activities" - as detailed in "Regulations governing the attainment of doctoral degree", §22, Maastricht University.

Within this thesis, I presented fundamental applied research in the field of RF engineering with direct applications for enabling cognitive neuroscience research. In particular, I used innovative RF receive and transmit coil layout methods to design and validate RF coils for both in-vivo (Chapter 2) and ex-vivo (Chapters 3-5) use, at UHF strengths of $7 \mathrm{~T}$ and 9.4T respectively. These developed coils, therefore, are not only relevant for researchers employing MR methods to study brain structure, function and connectivity, but also for those utilizing exvivo imaging methods such as Diffusion Weighted (DWI) and Susceptibility Weighted Imaging (SWI) to further probe and understand the neuronal basis of connectivity in the human brain. Over the course of my work at FPN/CN, researchers have stressed upon the need for customised RF coil hardware for their high-resolution imaging needs, and the work detailed in this thesis describes the development of such coils.

\section{T: From research flagship to clinical trendsetter}

MRI is a non-invasive imaging method that has established itself as an invaluable imaging modality for both clinical and research purposes. While field strengths of $1.5 \mathrm{~T}$ and $3 \mathrm{~T}$ are the norm in a clinical setting, a lot of cognitive neuroscience research focuses on imaging at 7T [11, 14]. Recent policy updates (as of October 2017) have cleared the way for the clinical use of 7T scanners (Siemens Magnetom "Terra") through 
FDA (US Food and Drug Administration) and CE (Conformité Européenne) approvals. The gains afforded at these higher field strengths include more than twice as much SNR per unit time, resulting in higher spatial resolutions and decreased scanning times - thereby enhancing clinical acquisitions and potentially reducing patient discomfort and time spent for an examination. Evidence on the benefits of high-field imaging for clinical purposes has been published by Zwanenburg et al. [13], Van der Kolk et al [16] and Trattnig et al [160], among others. With the increased Signal-to-Noise and Contast-to-Noise ratios afforded through 7T imaging, pathologies such as multiple sclerosis (MS), brain tumours and cerebrovascular diseases can be easily identified. With the achievable spatial resolution at 7T MRI approaching the scale of pathologies involved in neurodegenerative diseases such as Alzheimer's (AD) and Huntington's diseases (HD), further insight into these afflictions can be gleaned [161]. The ability to garner additional pathophysiological information can result in quicker diagnosis and start of treatment, while also providing insight on the development of new treatments for said diseases.

In this context, the availability of customised RF hardware is of primary importance when it comes to imaging specific anatomies, such that the gains afforded at high field strengths can be effectively harnessed by the researcher and/or clinician. For instance, the RF head coil detailed in Chapter 2 can not only be used for conducting highresolution, visual fMRI experiments, but can also help in diagnosing, for example, cortical blindness [162, 163] and occipital infarctions [164] in prospective patients. The same RF coil design can also be easily adapted to image other brain regions, such as the auditory and frontal lobes, while further modifications to similar coil geometries can help enhance clinical imaging at lower field strengths $(\leq 3 \mathrm{~T})$. With widescale clinical adoption of 7T systems on the horizon, novel RF receive and transmit coil designs such as that outlined in Chapter 2 of this thesis will play a crucial role in enabling high resolution, high throughput clinical datasets. 


\section{Neuroanatomy and histological imaging at UHF}

Diffusion magnetic resonance (dMRI) is a non-invasive imaging modality used to probe the connectivity and microstructure of the human brain, both through in-vivo and ex-vivo acquisition methods. A limiting factor for in-vivo dMRI is the average voxel size, which ranges between 1-3 mm, and for ex-vivo dMRI the same voxel size can be in the order of a few hundred micrometers [165].

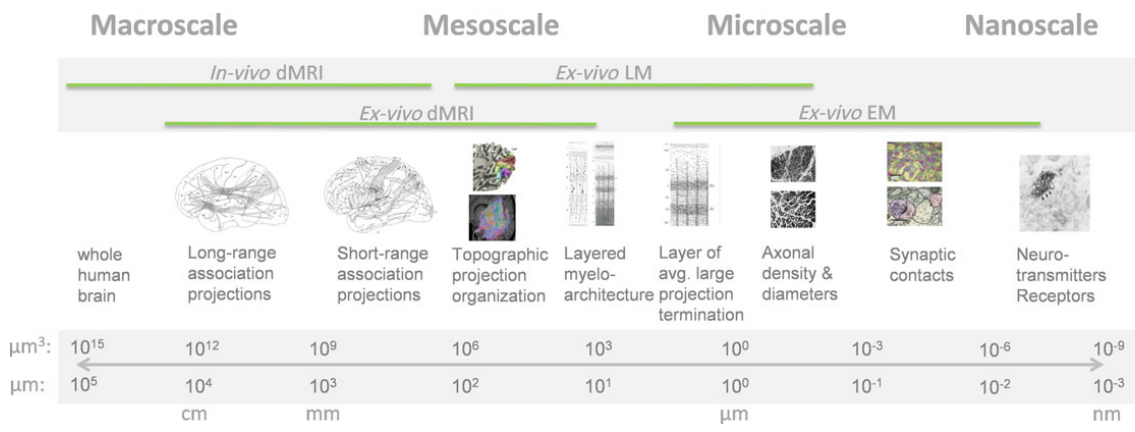

Figure 7.1: The multiscale nature of human structural brain connectivity and its measurement with different techniques. The measurement of the connectivity phenomena here refers to features directly resolved by the acquired spatial resolution of the technique (not by modeling the contrast over multiple measurements and indirectly inferring statistics of such features, as in microstructure modeling of diffusion MRI). dMRI, diffusion MRI; EM, electron microscopy; LM, light microscopy (Reproduced with permission from Roebroeck et al [165])

In-vivo dMRI has had a crucial role in mapping human macroscale connectomics, as evidenced through the Human Connectome Project [166] in which macroscopic human brain circuits have been mapped with in-vivo dMRI and tractography, along with fMRI measurements, across a large population of healthy adults, in order to create a " connectivity map" of the human brain and its various connections across cortices. At the other end of the spectrum, as shown in Figure 7.1, light microscopy (LM) techniques have helped study micro and nanoscale connectomics on small tissue samples. 
Ex-vivo dMRI plays an important part here. With its ability to achieve very high spatial resolutions (hundreds of $\mu \mathrm{m}$ 's) across FoVs larger than those possible with LM, it represents an ideal method for mapping mesoscale connectivity. At the same time, the connectivity and tractography maps generated from the high-resolution ex-vivo dMRI datasets need to be validated using histological techniques on the imaged sample, such that the different processing techniques implemented can be carefully evaluated and compared. In this regard, it is extremely important to not only have access to high-field MRI scanners fitted with strong gradients, but also to specific RF coil hardware relevant to the geometry of the sample being imaged. As detailed in Chapters 3-5, close-fitting, conformal phased array coil geometries provide extended coverage of the entire sample and the small-diameter coil elements enable high SNR near the coil surface (and by extension in the gray matter of the tissue under investigation, as the cortical layer is closest to the coil elements). We were able to achieve datasets with resolutions of upto $60 \mu \mathrm{m}$ isotropic for ex-vivo anatomical images and upto $200 \mu \mathrm{m}$ for quantitative acquisitions for relatively large tissue samples (upto $1 \mathrm{~cm}$ thick). The tractography results garnered from these datasets can then be validated by performing LM histological analysis on the same tissue sample, providing researchers with a robust, ground-truth measurement when comparing approaches to diffusion modelling.

Only a handful of research centers have access to a 9.4T human scanner, while many others have access to 7T human and 9.4T narrow-bore, pre-clinical (or animal) scanners. The work detailed in this thesis can be easily adapted to function across a variety of scanner configurations, thereby providing researchers with a solid RF platform for their high-resolution imaging needs. 
Acknowledgments 
I'd like to start off by expressing my deepest gratitude for having been given the opportunity to pursue my research work here at Maastricht University. The fact that the university houses one of the very few $9.4 \mathrm{~T}$ human scanners in the world was reason enough for me to pack my bags and make the trip from "sunny" Freiburg to the lands-of-alwaysovercast. Upon arrival, I was soon introduced to the convivial people here, working tirelessly to further the cause of UHF brain research at the highest level.

It is no surprise that these very same people seem to draw inspiration from my (and theirs too) supervisor, Rainer. He is perhaps the most easy-going, affable, accommodating, Tesla-driving department head I've come across. His passion for everything related to science and technology is infectious, and his constant support and encouragement helped me though some tough and uncertain times. Rainer, thank you for your belief in me and for helping me cross this threshold in my life. I'm already looking forward to the new in-vivo coil projects we discussed some time ago!

To my co-supervisors Alard and Gregor, thank you for everything. It is difficult to condense $5+$ years of work into a couple of lines of acknowledgement and appreciation, but I'd still like to thank you both for all that you've taught me and helped me with.

Alard, in your capacity as my daily supervisor, you encouraged me to delve beyond my comfort zone so that I could push myself further. And push we did. I remember the first $100 \mu \mathrm{m}$ feline brain dataset on the 9.4T, and how excited we were at the possibility of extending it to larger brain samples. I also thank you for your sustained belief in me and for motivating me, even on occasions where I had convinced myself that my work was not up to scratch. I am proud of what we have achieved together, and I hope in earnest that the ex-vivo coils continue to deliver great datasets, unabated, for as long as they're able to or until the next great RF coil comes around! 
Gregor, I owe much of the same to you. You were able to correctly evaluate my strengths and weaknesses and helped me work on them. Those 3 months we spent huddled in the RF lab working on the Visual Arc remains one of the fondest memories of my time here. While we did manage to blow up a number of preamplifiers on our very first test, it was a learning moment for me. I also fondly recall my time visiting you at CMRR, dealing with the insurmountable cold and being introduced to delicious lunch burritos. Thank you for the constant words of praise you'd send my way every time I'd write to you about a new set of results or a new coil - I hope there will be more to come.

To the "post-mortem" group - as we are still referred to - it's quite incredible that this heterogenous mixture of physicists, computer scientists and biologists work so well together, despite our idiosyncrasies and differences. Franc (aka Carajillo), thank you for all the hard work and dedication you put into every endeavour you undertake. You're going to mesmerise people with some of your upcoming work. To Robbert, thanks for all the help over the years - including designing the Maastricht Thesis Template which this dissertation uses. May your GPUs never tire and may our cryptocurrency ventures yield positive results in a century or so. To Sven, your clearing technique will see the light of day, and we shall then celebrate with some truly delicious Japanese single malt. Thanks for your patience and help in containing the several brain samples! To Anna, the de-facto matriarch of the group, thank you - for the many pep talks you gave me during the final months of the thesis, for the sessions at the 3rd floor pantry where we'd unload all our frustrations over a cup of coffee and for being the voice of reason - I wish you and your family all the best. To Sanne and Anna-Marie - late additions to the group, but it is made brighter by your presence. We really should check out the party scene in Nijmegen together. To Michael, the only guy I know who could legitimately audition for "The Most Interesting Man in the World" and nail it - thank you for always providing me with cogent and valuable advice. To Sri, my "bhai" and fellow Witcher + graphic novel enthusiast - may you be able to resolve laminar profiles using the Visual Arc. To Ron, thank 
you for the continued and unflinching support for everything electrical and RF - hope the engine blocks come through! To Riny and Christl you're the collective lifeblood of this department, always ready to help with a smile. Thank you for all that you do for us. And then there's everyone else - Valentin, Mario S, Mario-Archila, Sanae, Ben, Chris, Jan Z, Mehrdad, Ingo, Marian, Faruk, Gojko and Kamil - my time here was only helped by your presence, so big ups to you all!

Bogna, my partner and rock, who effortlessly props me up whenever I'm down - thank you for inhabiting this life with me. It would never be the same without you.

And finally, a thank you to my parents - Tapan and Sharmila - for all the sacrifices you made so that we could be where we are now, and to my sister Antara - for tolerating this brat of a brother. Love you all.

Shubharthi Sengupta

Maastricht 


\section{Curriculum Vitae}


Shubharthi was born on December 20, 1983, in Kolkata, India. While he was happy reading Tintin and speeding across imaginary racetracks in his Hot Wheels cars, he was nonetheless admitted to Don Bosco School, Park Circus, Kolkata where he received his secondary education (grade 91\%). In 2002, he started his undergraduate education at VIT University, India, graduating with a degree in Electronics and Communications Engineering. After working in the industry for 3 years, he made his way to sunny Freiburg, Germany to undertake a degree in Microsystems Engineering, minoring in Biomedical Instrumentation. His Master's thesis, titled " Development of an RF probe array for simultaneous Magnetic Resonance Imaging" was completed at IMTEK, University of Freiburg, under the supervision of Prof.dr. Jan Korvink. In 2012, he started his PhD studies at the Department of Cognitive Neuroscience under the guidance of Prof.dr. Rainer Goebel, Dr. Alard Roebroeck and Dr. Gregor Adriany. Shubharthi currently works as a Technical Developer at Scannexus BV and is still trying to get used to the infamous Dutch weather. 


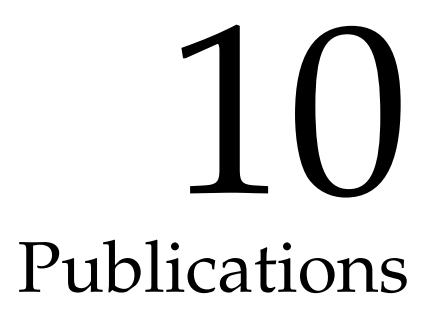





\section{Peer reviewed journal articles}

Sengupta, S., Roebroeck, A., Kemper, V.G., Poser, B.A., Zimmermann, J., Goebel R., and Adriany, G. (2016) A specialized multi-transmit head coil for high resolution fMRI of the human visual cortex at 7T. PLoS ONE 11(12): e0165418. doi:10.1371/journal.pone.0165418

Sengupta, S., Fritz, F.J., Harms, R., Hildebrand, S., Tse, D., Poser, B.A., Goebel, R., and Roebroeck, A. (2018). High resolution anatomical and quantitative MRI of the entire human occipital lobe ex vivo at $9.4 \mathrm{~T}$. NeuroImage, 168, 162-171. doi:10.1016/j.neuroimage.2017.03.039

Sengupta, S., Fritz, F.J., Caspers, S., Zilles, K., Lataster, A., Herrler, A., Tse, D.H.Y., Poser, B.A., and Roebroeck, A. (in preparation) High resolution MRI neuroanatomy in intact human brains post mortem with a specialized 9.4T RF coil

Kashyap, S., Ivanov, D., Havliček, M., Sengupta, S., Poser, B.A., and Uludağ, K. (accepted, in press) Resolving laminar activation in human V1 using ultra-high spatial resolution fMRI at 7T. Scientific Reports

Fritz, F.J., Sengupta, S., Harms, R.L., Tse, D.H.Y., Poser, B.A., and Roebroeck, A. (under review) Ultra-high resolution and multi-shell diffusion MRI of intact post mortem human brains using $k_{T}$-dSTEAM at $9.4 \mathrm{~T}$

Schneider, M., Marquardt, I., Sengupta, S., de Martino, F., and Goebel, $R$. (in preparation) The effect of visual motion direction on population receptive field estimates. 


\section{Conference procceedings}

Sengupta, S., Adriany, G., Kemper, V., Zimmermann, J., Goebel, R., and Roebroeck, A. An open 4ch. transmit / $16 \mathrm{ch}$. receive coil for high resolution occipital and temporal visual cortex imaging at 7T. In Proceedings of the 23rd ISMRM Annual Meeting, Toronto, Canada (2015) 1777.

Sengupta, S., van Zandvoort, M., Paes, D., Galuske, R., Goebel, R., and Roebroeck, R. Toward high resolution anatomical imaging of large ex vivo brain samples with specialized 9.4T RF coils. In Proceedings of the 23rd ISMRM Annual Meeting, Toronto, Canada (2015) 3480.

Roebroeck, A., Sengupta, S., Bastiani, M., Schillak, S., Tramm, B., Waks, M., Lataster, A., Herrler, A., Tse, D., and Poser, B.A. High resolution MRI neuroanatomy of the whole human brain post mortem with a specialized 9.4T RF-coil. Annual Meeting of the Organization for Human Brain Mapping, Honolulu (2015)

Sengupta, S., Hellenbrand, R., Finger, R., Wiggins, C., and Roebroeck, A. High resolution anatomical imaging of the human occipital lobe with a large ex-vivo 9.4T RF coil. In Proceedings of the 24th International Society for Magnetic Resonance in Medicine, Singapore (2016) 1163

Sengupta, S., Adriany, G., Kemper, V.G., Goebel, R., and Roebroeck, A. A larger subject field-of-view and denser coil arrays at UHF: What do we gain? In Proceedings of the 24th International Society for Magnetic Resonance in Medicine, Singapore (2016) 3520

Fritz, FJ., Foxley, S., Sengupta, S., Harms, R., Caspers, S., Zilles, K., Tse, D.H.Y., Poser, B., Miller, K.L., and Roebroeck, A. Whole human brain diffusion MRI at $450 \mu \mathrm{m}$ post mortem with dwSSFP and a specialized 9.4T RF-coil. In Proceedings of the 24th International Society for Magnetic Resonance in Medicine, Singapore (2016) 3379 
Sengupta, S., Fritz, F.J., Hellenbrand, R., Finger, R., Wiggins, C., and Roebroeck, A. A Modular RF Coil Platform for Ex-Vivo Imaging of Brain Slices at 9.4T. In Proceedings of the 25th International Society for Magnetic Resonance in Medicine, Honolulu (2017) 2413

Fritz, F.J., Tse, D.H.Y., Sengupta, S., Loderhose, T., Kraaijeveld, B., Caspers, S., Poser, B., and Roebroeck, A. Kt-dSTEAM: High resolution diffusion-weighted imaging of the ex vivo human brain using $B_{1}{ }^{+}$homogenized STEAM at 9.4T. In Proceedings of the 25th International Society for Magnetic Resonance in Medicine, Honolulu (2017) 0173

Sengupta, S., and Roebroeck, R. Dielectric pads for high-field MRI at 7T: a simulation study. In Proceedings of the 26th International Society for Magnetic Resonance in Medicine, Paris (2018) 4307

Fritz, F.J, Sengupta, S., Harms, R., Poser, B., Roebroeck, A. Ultra-high resolution multi-shell $\mathrm{dMRI}$ and tractography of the ex vivo human brain using kT-dSTEAM at 9.4T. In Proceedings of the 26th International Society for Magnetic Resonance in Medicine, Paris (2018) 0045

Kashyap, S., Ivanov, D., Sengupta, S., Poser, B., and Uludag, K. True laminar resolution $\mathrm{fMRI}$ of the human visual cortex at 7T. In Proceedings of the 26th International Society for Magnetic Resonance in Medicine, Paris (2018) 0394

Hildebrand, S., Schueth, A., Sengupta, S., Kiessling, A., Herrler, A., Galuske, R., and Roebroeck, A. A scalable processing pipeline for highthroughput optical clearing and labelling of very large formalin-fixed human brain samples. Program No. 170.07. 2018 Neuroscience Meeting Planner. San Diego, CA: Society for Neuroscience, 2018. Online 



\section{Bibliography}

[1] Rabi I. I., Zacharias J. R., Millman S., and Kusch P. A New Method of Measuring Nuclear Magnetic Moment. Physical Review, 53(4):318-318, feb 1938.

[2] Bloch F. Nuclear Induction. Physical Review, 70(7-8):460-474, oct 1946.

[3] Purcell E. M., Torrey H. C., and Pound R. V. Resonance Absorption by Nuclear Magnetic Moments in a Solid. Physical Review, 69(1-2):37-38, jan 1946.

[4] Damadian R. Tumor detection by nuclear magnetic resonance. Science (New York, N.Y.), 171(3976):1151-3, mar 1971.

[5] Lauterbur P. C. Magnetic resonance zeugmatography. Pure and Applied Chemistry, 40(1-2):149-157, jan 1974.

[6] Lauterbur P. C. Image formation by induced local interactions: Examples employing nuclear magnetic resonance. Nature, 242(5394):190-191, jul 1973.

[7] Mansfield P. and Grannell P. K. NMR 'diffraction' in solids? Journal of Physics C: Solid State Physics, 6(22):L422-L426, nov 1973. 
[8] Bottomley P., Hart H., Edelstein W., Schenck J., Smith L., Leue W., Mueller O., and Redington R. NMR imaging/spectroscopy system to study both anatomy and metabolism. The Lancet, 322(8344):273-274, jul 1983.

[9] Vaughan J. T., Garwood M., Collins C. M., Liu W., DelaBarre L., Adriany G., Andersen P., Merkle H., Goebel R., Smith M. B., and Ugurbil K. 7T vs. 4T: RF power, homogeneity, and signal-tonoise comparison in head images. Magnetic resonance in medicine, 46(1):24-30, jul 2001.

[10] Formisano E., Kim D. S., Di Salle F., Moortele P. F. van de, Ugurbil K., and Goebel R. Mirror-symmetric tonotopic maps in human primary auditory cortex. Neuron, 40(4):859-69, nov 2003.

[11] Polimeni J. R. and Uludağ K. Neuroimaging with ultra-high field MRI: Present and future. NeuroImage, 168:1-6, mar 2018.

[12] Vaughan T., DelaBarre L., Snyder C., Tian J., Akgun C., Shrivastava D., Liu W., Olson C., Adriany G., Strupp J., Andersen P., Gopinath A., Moortele P.-F. van de, Garwood M., and Ugurbil K. 9.4T human MRI: Preliminary results. Magnetic Resonance in Medicine, 56(6):1274-1282, dec 2006.

[13] Zwanenburg J. J. M., Kolk A. G. van der, and Luijten P. R. UltraHigh-Field MR Imaging: Research Tool or Clinical Need? PET clinics, 8(3):311-28, jul 2013.

[14] Balchandani P. and Naidich T. P. Ultra-High-Field MR Neuroimaging. AJNR. American journal of neuroradiology, 36(7):120415, jul 2015.

[15] Ladd M. E. High-Field-Strength Magnetic Resonance. Topics in Magnetic Resonance Imaging, 18(2):139-152, apr 2007.

[16] Kolk A. G. van der, Hendrikse J., Zwanenburg J. J., Visser F., and Luijten P. R. Clinical applications of 7T MRI in the brain. European Journal of Radiology, 82(5):708-718, may 2013. 
[17] Sodickson D. K. and Manning W. J. Simultaneous acquisition of spatial harmonics (SMASH): fast imaging with radiofrequency coil arrays. Magnetic resonance in medicine, 38(4):591-603, oct 1997.

[18] Pruessmann K. P., Weiger M., Scheidegger M. B., and Boesiger P. SENSE: Sensitivity encoding for fast MRI. Magnetic Resonance in Medicine, 42(5):952-962, 1999.

[19] Griswold M. A., Jakob P. M., Heidemann R. M., Nittka M., Jellus V., Wang J., Kiefer B., and Haase A. Generalized autocalibrating partially parallel acquisitions (GRAPPA). Magnetic resonance in medicine, 47(6):1202-10, jun 2002.

[20] Ackerman J. J., Grove T. H., Wong G. G., Gadian D. G., and Radda G. K. Mapping of metabolites in whole animals by 31P NMR using surface coils. Nature, 283(5743):167-70, jan 1980.

[21] Roemer P. B., Edelstein W. A., Hayes C. E., Souza S. P., and Mueller O. M. The NMR phased array. Magnetic Resonance in Medicine, 16(2):192-225, nov 1990.

[22] Raaijmakers A. J., Luijten P. R., and Berg C. A. van den. Dipole antennas for ultrahigh-field body imaging: a comparison with loop coils. NMR in biomedicine, (April), 2016.

[23] Adriany G., Van de Moortele P.-F., Wiesinger F., Moeller S., Strupp J. P., Andersen P., Snyder C., Zhang X., Chen W., Pruessmann K. P., Boesiger P., Vaughan T., and Uğurbil K. Transmit and receive transmission line arrays for 7 Tesla parallel imaging. Magnetic resonance in medicine : official journal of the Society of Magnetic Resonance in Medicine / Society of Magnetic Resonance in Medicine, 53(2):434-45, feb 2005.

[24] Hoult D. I. and Richards R. E. The Signal-to-Noise Ratio of the Nuclear Magnetic Resonance Experiment. 85:71-85, 1976.

[25] Hoult D. I. The principle of reciprocity in signal strength calculations?A mathematical guide, 2000. 
[26] Webb a. G. Radiofrequency microcoils for magnetic resonance imaging and spectroscopy. Journal of magnetic resonance (San Diego, Calif. : 1997), 229:55-66, apr 2013.

[27] Ong K. C., Wen H., Chesnick A. S., Duewell S., Jaffer F. A., and Balaban R. S. Radiofrequency shielding of surface coils at $4.0 \mathrm{~T}$. Journal of magnetic resonance imaging : JMRI, 5(6):773-7.

[28] Ali Caglar Özen. Novel MRI Technologies for Structural and Functional Imaging of Tissues with Ultra- short T2 Values. PhD thesis, 2017.

[29] Hoult D. I. Receiver Design for MR. In eMagRes, pages 1-21. John Wiley \& Sons, Ltd, Chichester, UK, mar 1996.

[30] Bomsdorf H., Helzel T., Kunz D., Röschmann P., Tschendel O., and Wieland J. Spectroscopy and imaging with a 4 tesla wholebody mr system. NMR in Biomedicine, 1(3):151-158, jun 1988.

[31] Sled J. G. and Pike G. B. Standing-wave and RF penetration artifacts caused by elliptic geometry: an electrodynamic analysis of MRI. IEEE transactions on medical imaging, 17(4):653-62, aug 1998.

[32] Wang J., Yang Q. X., Zhang X., Collins C. M., Smith M. B., Zhu X.H., Adriany G., Ugurbil K., and Chen W. Polarization of the RF field in a human head at high field: A study with a quadrature surface coil at 7.0 T. Magnetic Resonance in Medicine, 48(2):362369 , aug 2002.

[33] Glover G., Hayes C., Pelc N., Edelstein W., Mueller O., Hart H., Hardy C., O'Donnell M., and Barber W. Comparison of linear and circular polarization for magnetic resonance imaging. Journal of Magnetic Resonance (1969), 64(2):255-270, sep 1985.

[34] Keltner J. R., Carlson J. W., Roos M. S., Wong S. T., Wong T. L., and Budinger T. F. Electromagnetic fields of surface coil in vivo NMR at high frequencies. Magnetic resonance in medicine, 22(2):467-80, dec 1991. 
[35] Wald L. L. and Adalsteinsson E. Parallel-Excitation Techniques for Ultra-High-Field MRI. In Parallel Imaging in Clinical MR Applications, pages 511-521. Springer Berlin Heidelberg, Berlin, Heidelberg.

[36] Katscher U. and Börnert P. Parallel RF transmission in MRI. NMR in biomedicine, 19(3):393-400, may 2006.

[37] Webb A. G. and Collins C. M. Parallel transmit and receive technology in high-field magnetic resonance neuroimaging. International Journal of Imaging Systems and Technology, 20(1):2-13, feb 2010.

[38] Collins C. M., Liu W., Swift B. J., and Smith M. B. Combination of optimized transmit arrays and some receive array reconstruction methods can yield homogeneous images at very high frequencies. Magnetic resonance in medicine, 54(6):1327-32, dec 2005.

[39] Metzger G. J., Snyder C., Akgun C., Vaughan T., Ugurbil K., and Van de Moortele P.-F. Local B1+ shimming for prostate imaging with transceiver arrays at 7T based on subject-dependent transmit phase measurements. Magnetic resonance in medicine, 59(2):396-409, feb 2008.

[40] Hagen J. Radio-Frequency Electronics: Circuits and Applications. 2nd edition, 2009.

[41] Cloos M. A., Luong M., Ferrand G., Amadon A., Le Bihan D., and Boulant N. Local SAR reduction in parallel excitation based on channel-dependent Tikhonov parameters. Journal of Magnetic Resonance Imaging, 32(5):1209-1216, nov 2010.

[42] Homann H., Graesslin I., Eggers H., Nehrke K., Vernickel P., Katscher U., Dössel O., and Börnert P. Local SAR management by RF Shimming: a simulation study with multiple human body models. Magnetic Resonance Materials in Physics, Biology and Medicine, 25(3):193-204, jun 2012. 
[43] Hyde J. S., Jesmanowicz A., Froncisz W., Bruce Kneeland J., Grist T. M., and Campagna N. F. Parallel image acquisition from noninteracting local coils. Journal of Magnetic Resonance (1969), 70(3):512-517, dec 1986.

[44] Kokubunji T., Soka T., Kashiwa Y., Akishima E., and Katakura K. RF probe for MRI, 1994.

[45] Wu B., Zhang X., Qu P., and Shen G. X. Design of an inductively decoupled microstrip array at $9.4 \mathrm{~T}$. Journal of magnetic resonance (San Diego, Calif. : 1997), 182(1):126-32, sep 2006.

[46] Porter J. R., Wright S. M., and Reykowski A. A 16-element phased-array head coil. Magnetic Resonance in Medicine, 40(2):272-279, aug 1998.

[47] Hardy C. J., Giaquinto R. O., Piel J. E., Rohling K. W., Marinelli L., Blezek D. J., Fiveland E. W., Darrow R. D., and Foo T. K. F. 128-channel body MRI with a flexible high-density receiver-coil array. Journal of magnetic resonance imaging : JMRI, 28(5):1219-25, nov 2008.

[48] Wiggins G. C., Triantafyllou C., Potthast a., Reykowski a., Nittka M., and Wald L. L. 32-channel 3 Tesla receive-only phasedarray head coil with soccer-ball element geometry. Magnetic resonance in medicine: official journal of the Society of Magnetic Resonance in Medicine / Society of Magnetic Resonance in Medicine, 56(1):216-23, jul 2006.

[49] Keil B., Blau J. N., Biber S., Hoecht P., Tountcheva V., Setsompop K., Triantafyllou C., and Wald L. L. A 64-channel 3T array coil for accelerated brain MRI. Magnetic Resonance in Medicine, 70(1):248-258, 2013.

[50] Wiggins G. C., Polimeni J. R., Potthast A., Schmitt M., Alagappan V., and Wald L. L. 96-Channel receive-only head coil for 3 Tesla: design optimization and evaluation. Magnetic resonance in medicine, 62(3):754-62, sep 2009. 
[51] Schmitt M., Potthast A., Sosnovik D. E., Polimeni J. R., Wiggins G. C., Triantafyllou C., and Wald L. L. A 128-channel receive-only cardiac coil for highly accelerated cardiac MRI at 3 Tesla. Magnetic resonance in medicine, 59(6):1431-9, jun 2008.

[52] Deshmane A., Gulani V., Griswold M. A., and Seiberlich N. Parallel MR imaging. Journal of magnetic resonance imaging : JMRI, 36(1):55-72, jul 2012.

[53] NYU Center for Biomedical Imaging. G16.4427 Practical MRI 1. 2013.

[54] Kane Yee. Numerical solution of initial boundary value problems involving maxwell's equations in isotropic media. IEEE Transactions on Antennas and Propagation, 14(3):302-307, may 1966.

[55] International Electrotechnical Commission. IEC 60601-2-33:2010 - Medical electrical equipment - Part 2-33: Particular requirements for the basic safety and essential performance of magnetic resonance equipment for medical diagnosis, 2010.

[56] Sereno M., Dale A., Reppas J., Kwong K., Belliveau J., Brady T., Rosen B., and Tootell R. Borders of multiple visual areas in humans revealed by functional magnetic resonance imaging. Science, 268(5212):889-893, may 1995.

[57] Menon R. S. and Goodyear B. G. Submillimeter functional localization in human striate cortex using BOLD contrast at 4 Tesla: Implications for the vascular point-spread function. Magnetic Resonance in Medicine, 41(2):230-235, 1999.

[58] Wandell B. A., Dumoulin S. O., and Brewer A. A. Visual field maps in human cortex. Neuron, 56(2):366-83, oct 2007.

[59] Glasser M. F., Coalson T. S., Robinson E. C., Hacker C. D., Harwell J., Yacoub E., Ugurbil K., Andersson J., Beckmann C. F., Jenkinson M., Smith S. M., and Van Essen D. C. A multi-modal 
parcellation of human cerebral cortex. Nature, 536(7615):171-178, 2016.

[60] Turner R. and Geyer S. Introduction to the NeuroImage Special Issue: "In vivo Brodmann mapping of the human brain". Neurolmage, 93:155-156, jun 2014.

[61] Weiskopf N., Mohammadi S., Lutti A., and Callaghan M. F. Advances in MRI-based computational neuroanatomy: from morphometry to in-vivo histology. Current opinion in neurology, 28(4):313-22, aug 2015.

[62] Sengupta S., Roebroeck A., Kemper V. G., Poser B. A., Zimmermann J., Goebel R., and Adriany G. A specialized multi-transmit head coil for high resolution fMRI of the human visual cortex at 7T. PLoS ONE, 11(12):1-22, 2016.

[63] Ledden P. J., Mareyam a., Wang S., Gelderen P. V., and Duyn J. 32 Channel Receive-Only SENSE Array for Brain Imaging at 7T. Proceedings of the joint annual meeting ISMRM-ESMRMB, Berlin, Germany, 51(2004):242, 2007.

[64] Sengupta S., Adriany G., Kemper V. G., Zimmermann J., Goebel R., and Roebroeck A. An Open 4ch. Transmit / 16 Ch. Receive Coil for High Resolution Occipital and Temporal Visual Cortex Imaging at 7T. In Proceedings of the 23rd ISMRM Annual Meeting, Toronto, Canada., page 1777, 2015.

[65] Shajan G., Kozlov M., Hoffmann J., Turner R., Scheffler K., and Pohmann R. A 16-channel dual-row transmit array in combination with a 31-element receive array for human brain imaging at 9.4 T. Magnetic resonance in medicine : official journal of the Society of Magnetic Resonance in Medicine / Society of Magnetic Resonance in Medicine, 000, mar 2013.

[66] Wiggins G. C., Zhang B., Duan Q., Lattanzi R., Biber S., Stoeckel B., McGorty K., and Sodickson D. K. 7 Tesla Transmit- 
Receive Array for Carotid Imaging: Simulation and Experiment. Proc. Intl. Soc. Mag. Reson. Med. 17, 17:394, 2009.

[67] Orzada S., Maderwald S., Göricke S. L., Parohl N., Ladd S. C., Ladd M. E., and Quick H. H. Design and comparison of two eight-channel transmit/receive radiofrequency arrays for in vivo rodent imaging on a $7 \mathrm{~T}$ human whole-body MRI system. Medical physics, 37(5):2225-32, may 2010.

[68] Oezerdem C., Winter L., Graessl A., Paul K., Els A., Weinberger O., Rieger J., Kuehne A., Dieringer M., Hezel F., Voit D., Frahm J., and Niendorf T. 16-channel bow tie antenna transceiver array for cardiac MR at 7.0 tesla. Magnetic resonance in medicine, 00(February):1-13, 2015.

[69] Keil B., Wiggins G. C., Triantafyllou C., Wald L. L., Meise F. M., Schreiber L. M., Klose K. J., and Heverhagen J. T. A 20-channel receive-only mouse array coil for a $3 \mathrm{~T}$ clinical MRI system. Magnetic Resonance in Medicine, 66(2):584-595, 2011.

[70] Moortele P.-f. V. D., Snyder C., DelaBarre L., Adriany G., Vaughan T., and Ugurbil K. Calibration tools for RF shim at very high field with multiple element RF coils: from ultra fast local relative $\mathrm{B} 1$ phase to absolute magnitude B1 mapping. In Proceedings of the 15th ISMRM, Berlin, page 1676, 2007.

[71] Petridou N., Italiaander M., Bank B. L. van de, Siero J. C. W., Luijten P. R., and Klomp D. W. J. Pushing the limits of highresolution functional MRI using a simple high-density multielement coil design. NMR in Biomedicine, 26(1):65-73, 2013.

[72] Lattanzi R. and Sodickson D. K. Ideal current patterns yielding optimal signal-to-noise ratio and specific absorption rate in magnetic resonance imaging: computational methods and physical insights. Magnetic resonance in medicine, 68(1):286-304, jul 2012.

[73] Wald L. L., Carvajal L., Moyher S. E., Nelson S. J., Grant P. E., Barkovich A. J., and Vigneron D. B. Phased array detectors and 
an automated intensity-correction algorithm for high-resolution MR imaging of the human brain. Magnetic resonance in medicine, 34(3):433-9, sep 1995.

[74] Wright S. M. and Wald L. L. Theory and application of array coils in MR spectroscopy. NMR in biomedicine, 10(8):394-410, dec 1997.

[75] De Zwart J. a., Ledden P. J., Kellman P., Van Gelderen P., and Duyn J. H. Design of a SENSE-optimized high-sensitivity MRI receive coil for brain imaging. Magnetic Resonance in Medicine, 47(6):1218-1227, 2002.

[76] Adriany G., Waks M., Tramm B., Schillak S., Yacoub E., Martino F. D., Van de Moortele P.-F., Naselaris T., Olman C., Vaughan T., and Ugurbil K. An Open Faced 4 ch. Loop Transmit $/ 16$ ch. Receive Array Coil for HiRes fMRI at 7 Tesla. Proceedings of the 20th Annual Meeting of the ISMRM, 20:429, 2012.

[77] Reykowski A., Wright S. M., and Porter J. R. Design of Matching Networks for Low Noise Preamplifiers. Magnetic resonance in medicine, 33(6):848-852, jun 1995.

[78] Possanzini C. and Boutelje M. Influence of magnetic field on preamplifiers using GaAs FET technology. In Proceedings 16th Scientific Meeting, International Society for Magnetic Resonance in Medicine, volume Toronto, page 1123, 2008.

[79] Kozlov M. and Turner R. Fast MRI coil analysis based on 3-D electromagnetic and RF circuit co-simulation. Journal of magnetic resonance (San Diego, Calif. : 1997), 200(1):147-52, sep 2009.

[80] Yarnykh V. L. Actual flip-angle imaging in the pulsed steady state: a method for rapid three-dimensional mapping of the transmitted radiofrequency field. Magnetic resonance in medicine, 57(1):192-200, jan 2007. 
[81] Moeller S., Auerbach E. J., Moortele P.-F. van de, Adriany G., and Ugurbil K. fMRI with 16 fold reduction using multibanded multislice sampling. In Proc. Int. Soc. Magn. Reson. Med, volume 16, page 2366, 2008.

[82] Shajan G., Mirkes C., Buckenmaier K., Hoffmann J., Pohmann R., and Scheffler K. Three-layered radio frequency coil arrangement for sodium MRI of the human brain at 9.4 Tesla. Magnetic Resonance in Medicine, 916:906-916, 2015.

[83] Robson P. M., Grant A. K., Madhuranthakam A. J., Lattanzi R., Sodickson D. K., and McKenzie C. A. Comprehensive quantification of signal-to-noise ratio and g-factor for image-based and k-space-based parallel imaging reconstructions. Magnetic resonance in medicine, 60(4):895-907, oct 2008.

[84] Walsh D. O., Gmitro A. F., and Marcellin M. W. Adaptive reconstruction of phased array MR imagery. Magnetic resonance in medicine, 43(5):682-90, may 2000.

[85] Avdievich N., Giapitzakis I., and Henning A. Asymmetric Transceiver Phased Array for Functional Imaging and Spectroscopy of the Visual Cortex at 9.4 T. In Proceedings of the 23rd ISMRM Annual Meeting, Toronto, Canada, page 3177, 2015.

[86] Shmuel A., Augath M., Oeltermann A., and Logothetis N. K. Negative functional MRI response correlates with decreases in neuronal activity in monkey visual area V1. Nature neuroscience, 9(4):569-77, apr 2006.

[87] Sengupta S., Fritz F. J., Harms R. L., Hildebrand S., Tse D. H. Y., Poser B. A., Goebel R., and Roebroeck A. High resolution anatomical and quantitative MRI of the entire human occipital lobe ex vivo at 9.4T. NeuroImage, 168:162-171, 2017.

[88] Keil B., Alagappan V., Mareyam A., McNab J. a., Fujimoto K., Tountcheva V., Triantafyllou C., Dilks D. D., Kanwisher N., Lin W., Grant P. E., and Wald L. L. Size-optimized 32-channel 
brain arrays for $3 \mathrm{~T}$ pediatric imaging. Magnetic Resonance in Medicine, 66(6):1777-1787, 2011.

[89] Duyn J. H., Gelderen P. van, Li T.-Q., Zwart J. A. de, Koretsky A. P., and Fukunaga M. High-field MRI of brain cortical substructure based on signal phase. Proceedings of the National Academy of Sciences of the United States of America, 104(28):11796801, jul 2007.

[90] Glasser M. F. and Van Essen D. C. Mapping human cortical areas in vivo based on myelin content as revealed by T1- and T2weighted MRI. The Journal of neuroscience : the official journal of the Society for Neuroscience, 31(32):11597-616, aug 2011.

[91] Barazany D. and Assaf Y. Visualization of cortical lamination patterns with magnetic resonance imaging. Cerebral cortex (New York, N.Y. : 1991), 22(9):2016-23, sep 2012.

[92] Dick F., Tierney A. T., Lutti A., Josephs O., Sereno M. I., and Weiskopf N. In vivo functional and myeloarchitectonic mapping of human primary auditory areas. The Journal of neuroscience : the official journal of the Society for Neuroscience, 32(46):16095-105, nov 2012.

[93] Sereno M. I., Lutti A., Weiskopf N., and Dick F. Mapping the human cortical surface by combining quantitative $\mathrm{T}(1)$ with retinotopy. Cerebral cortex (New York, N.Y. : 1991), 23(9):2261-8, sep 2013.

[94] De Martino F., Moerel M., Xu J., Moortele P.-F. van de, Ugurbil K., Goebel R., Yacoub E., and Formisano E. High-Resolution Mapping of Myeloarchitecture In Vivo: Localization of Auditory Areas in the Human Brain. Cerebral cortex (New York, N.Y. : 1991), 25(10):3394-405, oct 2015.

[95] Lutti A., Dick F., Sereno M. I., and Weiskopf N. Using highresolution quantitative mapping of $\mathrm{R} 1$ as an index of cortical myelination. Neurolmage, 93 Pt 2:176-88, jun 2014. 
[96] Stüber C., Morawski M., Schäfer A., Labadie C., Wähnert M., Leuze C., Streicher M., Barapatre N., Reimann K., Geyer S., Spemann D., and Turner R. Myelin and iron concentration in the human brain: a quantitative study of MRI contrast. NeuroImage, 93 Pt 1:95-106, jun 2014.

[97] Deistung A., Schäfer A., Schweser F., Biedermann U., Turner R., and Reichenbach J. R. Toward in vivo histology: a comparison of quantitative susceptibility mapping (QSM) with magnitude, phase-, and R2*-imaging at ultra-high magnetic field strength. NeuroImage, 65:299-314, jan 2013.

[98] Truong T.-K., Guidon A., and Song A. W. Cortical depth dependence of the diffusion anisotropy in the human cortical gray matter in vivo. PloS one, 9(3):e91424, 2014.

[99] Dinse J., Waehnert M., Tardif C. L., Schäfer A., Geyer S., Turner R., and Bazin P.-L. A histology-based model of quantitative T1 contrast for in-vivo cortical parcellation of highresolution 7 Tesla brain MR images. Medical image computing and computer-assisted intervention : MICCAI ... International Conference on Medical Image Computing and Computer-Assisted Intervention, 16(Pt 2):51-8, 2013.

[100] Geyer S., Weiss M., Reimann K., Lohmann G., and Turner R. Microstructural Parcellation of the Human Cerebral Cortex - From Brodmann's Post-Mortem Map to in vivo Mapping with HighField Magnetic Resonance Imaging. Frontiers in human neuroscience, 5:19, 2011.

[101] Augustinack J. C., Huber K. E., Stevens A. A., Roy M., Frosch M. P., Kouwe A. J. W. van der, Wald L. L., Van Leemput K., McKee A. C., and Fischl B. Predicting the location of human perirhinal cortex, Brodmann's area 35, from MRI. NeuroImage, 64(1):32-42, 2013.

[102] Augustinack J. C., Van Der Kouwe A. J. W., and Fischl B. Medial 
temporal cortices in ex vivo magnetic resonance imaging. Journal of Comparative Neurology, 521(18):4177-4188, 2013.

[103] Augustinack J. C., Magnain C., Reuter M., Kouwe A. J. W. van der, Boas D., and Fischl B. MRI parcellation of ex vivo medial temporal lobe. NeuroImage, 93:252-259, 2014.

[104] Waehnert M. D., Dinse J., Weiss M., Streicher M. N., Waehnert P., Geyer S., Turner R., and Bazin P.-L. Anatomically motivated modeling of cortical laminae. NeuroImage, 93 Pt 2:210-20, jun 2014.

[105] Modo M., Hitchens T. K., Liu J. R., and Richardson R. M. Detection of aberrant hippocampal mossy fiber connections: Ex vivo mesoscale diffusion MRI and microtractography with histological validation in a patient with uncontrolled temporal lobe epilepsy. Human brain mapping, 37(2):780-95, feb 2016.

[106] Glover P. M., Bowtell R. W., Brown G. D., and Mansfield P. A microscope slide probe for high resolution imaging at 11.7 Tesla. Magnetic resonance in medicine, 31(4):423-8, apr 1994.

[107] Fatterpekar G. M., Naidich T. P., Delman B. N., Aguinaldo J. G., Gultekin S. H., Sherwood C. C., Hof P. R., Drayer B. P., and Fayad Z. A. Cytoarchitecture of the human cerebral cortex: MR microscopy of excised specimens at 9.4 Tesla. AJNR. American journal of neuroradiology, 23(8):1313-21, sep 2002.

[108] Roebroeck A., Galuske R., Formisano E., Chiry O., Bratzke H., Ronen I., Kim D.-s., and Goebel R. High-resolution diffusion tensor imaging and tractography of the human optic chiasm at 9.4 T. NeuroImage, 39(1):157-68, jan 2008.

[109] Utz M. and Monazami R. Nuclear magnetic resonance in microfluidic environments using inductively coupled radiofrequency resonators. Journal of magnetic resonance (San Diego, Calif. : 1997), 198(1):132-6, may 2009. 
[110] Nabuurs R. J. a., Hegeman I., Natté R., Duinen S. G. van, Buchem M. a. van, Weerd L. van der, and Webb A. G. High-field MRI of single histological slices using an inductively coupled, self-resonant microcoil: application to ex vivo samples of patients with Alzheimer's disease. NMR in biomedicine, 24(4):351-7, may 2011.

[111] Seehaus A. K., Roebroeck A., Chiry O., Kim D.-S., Ronen I., Bratzke H., Goebel R., and Galuske R. A. W. Histological validation of DW-MRI tractography in human postmortem tissue. Cerebral cortex (New York, N.Y. : 1991), 23(2):442-50, feb 2013.

[112] Aggarwal M., Nauen D. W., Troncoso J. C., and Mori S. Probing region-specific microstructure of human cortical areas using high angular and spatial resolution diffusion MRI. NeuroImage, 105:198-207, jan 2015.

[113] Calabrese E., Hickey P., Hulette C., Zhang J., Parente B., Lad S. P., and Johnson G. A. Postmortem diffusion MRI of the human brainstem and thalamus for deep brain stimulator electrode localization. Human brain mapping, 36(8):3167-78, aug 2015.

[114] Weiss M., Alkemade A., Keuken M. C., Múller-Axt C., Geyer S., Turner R., and Forstmann B. U. Spatial normalization of ultrahigh resolution $7 \mathrm{~T}$ magnetic resonance imaging data of the postmortem human subthalamic nucleus: a multistage approach. Brain structure \& function, 220(3):1695-703, 2015.

[115] Augustinack J. C., Kouwe A. J. W. van der, Blackwell M. L., Salat D. H., Wiggins C. J., Frosch M. P., Wiggins G. C., Potthast A., Wald L. L., and Fischl B. R. Detection of entorhinal layer II using 7Tesla [corrected] magnetic resonance imaging. Annals of neurology, 57(4):489-94, apr 2005.

[116] Keil B. and Wald L. L. Massively parallel MRI detector arrays. Journal of magnetic resonance (San Diego, Calif. : 1997), 229:75-89, apr 2013. 
[117] Cusack R. and Papadakis N. New robust 3-D phase unwrapping algorithms: application to magnetic field mapping and undistorting echoplanar images. NeuroImage, 16(3 Pt 1):754-64, jul 2002.

[118] Nehrke K. and Bornert P. DREAM-a novel approach for robust, ultrafast, multislice B1 mapping. Magnetic resonance in medicine, 68(5):1517-26, nov 2012.

[119] Setsompop K., Wald L. L., Alagappan V., Gagoski B. A., and Adalsteinsson E. Magnitude least squares optimization for parallel radio frequency excitation design demonstrated at 7 Tesla with eight channels. Magnetic resonance in medicine, 59(4):908-15, apr 2008.

[120] Tse D. H. Y., Wiggins C. J., Ivanov D., Brenner D., Hoffmann J., Mirkes C., Shajan G., Scheffler K., Uludağ K., and Poser B. A. Volumetric imaging with homogenised excitation and static field at 9.4 T. Magma (New York, N.Y.), 29(3):333-45, jun 2016.

[121] Cloos M. A., Boulant N., Luong M., Ferrand G., Giacomini E., Le Bihan D., and Amadon A. kT -points: short three-dimensional tailored RF pulses for flip-angle homogenization over an extended volume. Magnetic resonance in medicine, 67(1):72-80, jan 2012.

[122] Triantafyllou C., Polimeni J. R., and Wald L. L. Physiological noise and signal-to-noise ratio in fMRI with multi-channel array coils. NeuroImage, 55(2):597-606, mar 2011.

[123] Harms H., Santis S. de, Bastiani M., Goebel R., and Roebroeck A. Diffusion microstructure modelling using a modular and extensible GPU accelerated toolkit. In Proceedings of the 23rd ISMRM Annual Meeting, Toronto, Canada, page 3656, 2015.

[124] Peters A. M., Brookes M. J., Hoogenraad F. G., Gowland P. A., Francis S. T., Morris P. G., and Bowtell R. T2* measurements 
in human brain at 1.5, 3 and 7 T. Magnetic resonance imaging, 25(6):748-53, jul 2007.

[125] Fukunaga M., Li T.-Q., Gelderen P. van, Zwart J. A. de, Shmueli K., Yao B., Lee J., Maric D., Aronova M. A., Zhang G., Leapman R. D., Schenck J. F., Merkle H., and Duyn J. H. Layerspecific variation of iron content in cerebral cortex as a source of MRI contrast. Proceedings of the National Academy of Sciences of the United States of America, 107(8):3834-9, feb 2010.

[126] Lee J., Shmueli K., Kang B.-T., Yao B., Fukunaga M., Gelderen P. van, Palumbo S., Bosetti F., Silva A. C., and Duyn J. H. The contribution of myelin to magnetic susceptibilityweighted contrasts in high-field MRI of the brain. NeuroImage, 59(4):3967-75, feb 2012.

[127] Reeves C., Tachrount M., Thomas D., Michalak Z., Liu J., Ellis M., Diehl B., Miserocchi A., McEvoy A. W., Eriksson S., Yousry T., and Thom M. Combined Ex Vivo 9.4T MRI and Quantitative Histopathological Study in Normal and Pathological Neocortical Resections in Focal Epilepsy. Brain pathology (Zurich, Switzerland), 26(3):319-33, may 2016.

[128] Fernández-Seara M. A. and Wehrli F. W. Postprocessing technique to correct for background gradients in image-based $\mathrm{R}^{*}(2)$ measurements. Magnetic resonance in medicine, 44(3):358-66, sep 2000 .

[129] Dell'Acqua F., Bodi I., Slater D., Catani M., and Modo M. MR diffusion histology and micro-tractography reveal mesoscale features of the human cerebellum. Cerebellum (London, England), 12(6):923-31, dec 2013.

[130] Kleinnijenhuis M., Zerbi V., Küsters B., Slump C. H., Barth M., and van Cappellen van Walsum A.-M. Layer-specific diffusion weighted imaging in human primary visual cortex in vitro. Cortex; a journal devoted to the study of the nervous system and behavior, 49(9):2569-82, oct 2013. 
[131] Leuze C. W. U., Anwander A., Bazin P.-L., Dhital B., Stüber C., Reimann K., Geyer S., and Turner R. Layer-specific intracortical connectivity revealed with diffusion MRI. Cerebral cortex (New York, N.Y. : 1991), 24(2):328-39, feb 2014.

[132] Leergaard T. B., White N. S., Crespigny A. de, Bolstad I., D'Arceuil H., Bjaalie J. G., and Dale A. M. Quantitative histological validation of diffusion MRI fiber orientation distributions in the rat brain. PloS one, 5(1):e8595, jan 2010.

[133] Axer M., Grässel D., Kleiner M., Dammers J., Dickscheid T., Reckfort J., Hütz T., Eiben B., Pietrzyk U., Zilles K., and Amunts K. High-resolution fiber tract reconstruction in the human brain by means of three-dimensional polarized light imaging. Frontiers in neuroinformatics, 5:34, 2011.

[134] Budde J., Shajan G., Schef K., and Pohmann R. NeuroImage Ultra-high resolution imaging of the human brain using acquisition-weighted imaging at 9 . 4 T. 71:2-8, 2013.

[135] Magnain C., Augustinack J. C., Reuter M., Wachinger C., Frosch M. P., Ragan T., Akkin T., Wedeen V. J., Boas D. A., and Fischl B. Blockface histology with optical coherence tomography: a comparison with Nissl staining. NeuroImage, 84:524-33, jan 2014.

[136] Khan A. R., Cornea A., Leigland L. A., Kohama S. G., Jespersen S. N., and Kroenke C. D. 3D structure tensor analysis of light microscopy data for validating diffusion MRI. NeuroImage, 111:192-203, may 2015.

[137] Seehaus A., Roebroeck A., Bastiani M., Fonseca L., Bratzke H., Lori N., Vilanova A., Goebel R., and Galuske R. Histological validation of high-resolution DTI in human post mortem tissue. Frontiers in neuroanatomy, 9:98, 2015.

[138] Sengupta S., Fritz F. J., Caspers S., Zilles K., Lataster A., Herrler A., Tse D. H. Y., Poser B. A., and Roebroeck A. High reso- 
lution MRI neuroanatomy in intact humans brain post mortem with a specialized 9.4T RF-coil. (in prep).

[139] Turner R. and Geyer S. Introduction to the NeuroImage special issue: "In vivo Brodmann mapping of the human brain". NeuroImage, 93 Pt 2:155-6, jun 2014.

[140] Miller K. L., Stagg C. J., Douaud G., Jbabdi S., Smith S. M., Behrens T. E., Jenkinson M., Chance S. A., Esiri M. M., Voets N. L., Jenkinson N., Aziz T. Z., Turner M. R., JohansenBerg H., and McNab J. A. Diffusion imaging of whole, postmortem human brains on a clinical MRI scanner. NeuroImage, 57(1):167-181, jul 2011.

[141] Foxley S., Jbabdi S., Clare S., Lam W., Ansorge O., Douaud G., and Miller K. Improving diffusion-weighted imaging of postmortem human brains: SSFP at 7 T. NeuroImage, 102 Pt 2:579-89, nov 2014.

[142] Orton M. R., Collins D. J., Koh D.-M., and Leach M. O. Improved intravoxel incoherent motion analysis of diffusion weighted imaging by data driven Bayesian modeling. Magnetic resonance in medicine, 71(1):411-20, jan 2014.

[143] Mareyam A., Polimeni J. R., and al E. Array coil and sample preparation and support system for whole brain ex vivo imaging at $100 \mu \mathrm{m}$. In Proceedings of the 23rd ISMRM Annual Meeting, Toronto, Canada, page 3130, 2015.

[144] Yang S., Yang Z., Fischer K., Zhong K., Stadler J., Godenschweger F., Steiner J., Heinze H.-J., Bernstein H.-G., Bogerts B., Mawrin C., Reutens D. C., Speck O., and Walter M. Integration of ultra-high field MRI and histology for connectome based research of brain disorders. Frontiers in neuroanatomy, 7:31, 2013.

[145] Chung S., Kim D., Breton E., and Axel L. Rapid B1+ mapping using a preconditioning RF pulse with TurboFLASH readout. Magnetic resonance in medicine, 64(2):439-46, aug 2010. 
[146] Barmet C., Zanche N. D., and Pruessmann K. P. Spatiotemporal magnetic field monitoring for MR. Magnetic Resonance in Medicine, 60(1):187-197, jul 2008.

[147] Sengupta S., Fritz F. J., Hellenbrand R., Finger R., Wiggins C., and Roebroeck A. A modular RF coil platform for ex-vivo imaging of brain slices at 9.4T. In Proceedings of the 25th Annual Meeting of ISMRM, Honolulu, USA, page 2413, 2017.

[148] Gruschke O. G., Baxan N., Clad L., Kratt K., Elverfeldt D. von, Peter A., Hennig J., Badilita V., Wallrabe U., and Korvink J. G. Lab on a chip phased-array MR multi-platform analysis system. Lab on a Chip, 12(3):495, 2012.

[149] Quester R. and Schröder R. The shrinkage of the human brain stem during formalin fixation and embedding in paraffin. Journal of neuroscience methods, 75(1):81-9, jul 1997.

[150] Mouritzen Dam A. Shrinkage of the brain during histological procedures with fixation in formaldehyde solutions of different concentrations. Journal fur Hirnforschung, 20(2):115-9, 1979.

[151] Meadowcroft M. D., Zhang S., Liu W., Bu S. P., Connor J. R., Collins C. M., Smith M. B., and Yang Q. X. Direct magnetic resonance imaging of histological tissue samples at 3.0T. Magnetic Resonance in Medicine, 57(5):835-841, 2007.

[152] Hildebrand S., Schueth A., Herrler A., and Galuske R. Scalable cytoarchitectonic characterization of large intact human neocortex samples. biorxiv, 274985, 2018.

[153] Hoang D. M., Voura E. B., Zhang C., Fakri-Bouchet L., and Wadghiri Y. Z. Evaluation of coils for imaging histological slides: Signal-to-noise ratio and filling factor. Magnetic resonance in medicine : official journal of the Society of Magnetic Resonance in Medicine / Society of Magnetic Resonance in Medicine, 1943:19321943, jul 2013. 
[154] Beckett A., Vu A., Keil B., Setsompop K., Wald L. L., Schillak S., and Feinberg D. Assessment of coil arrays with small loop diameter at 7T for micron-scale resolution fMRI of human neocortex. In Proceedings of the 24th Annual Meeting of ISMRM, Singapore, page 3519, 2016.

[155] Beckett A., Vu A., Schillak S., Wald L. L., and Feinberg D. A high density 24 channel array coil extendable to 48 channels for human cortical MRI at 7T. In Proceedings of the 25th Annual Meeting of ISMRM, Honolulu, USA, page 2654, 2017.

[156] Kashyap S., Ivanov D., Sengupta S., Poser B. A., and Uludağ K. True laminar resolution fMRI of the human visual cortex at 7T. In Proceedings of the 26th ISMRM, Paris (2018) \#0394.

[157] Zhang B., Chen G., Cloos M., Yu Z., Walczyk J., Collins C., Brown R., Lattanzi R., Sodickson D., and Wiggins G. 29-Channel receive-only dense dipole head array for 7T MRI. In 2017 International Conference on Electromagnetics in Advanced Applications (ICEAA), pages 1624-1627. IEEE, sep 2017.

[158] Duan Q., Nair G., Gudino N., Zwart J. A. de, Gelderen P. van, Murphy-Boesch J., Reich D. S., Duyn J. H., and Merkle H. A 7T spine array based on electric dipole transmitters. Magnetic resonance in medicine, 74(4):1189-97, oct 2015.

[159] Farhat S. S., Lee D. J., Costigan C., Gowland C. A., and Glover P. M. A dipole antenna for pelvis imaging at 7T. In Proceedings of the 20th Annual Meeting of ISMRM, Melbourne, Australia, page 2643, 2012.

[160] Trattnig S., Springer E., Bogner W., Hangel G., Strasser B., Dymerska B., Cardoso P. L., and Robinson S. D. Key clinical benefits of neuroimaging at $7 \mathrm{~T}$. NeuroImage, 168:477-489, mar 2018.

[161] McKiernan E. F. and O'Brien J. T. 7T MRI for neurodegenerative 
dementias in vivo: a systematic review of the literature. Journal of Neurology, Neurosurgery \& Psychiatry, 88(7):564-574, jul 2017.

[162] Zwicker J. C. and Sila C. A. MRI findings in a case of transient cortical blindness after cardiac catheterization. Catheterization and cardiovascular interventions : official journal of the Society for Cardiac Angiography \& Interventions, 57(1):47-9, sep 2002.

[163] Saigal G., Bhatia R., Bhatia S., and Wakhloo A. K. MR findings of cortical blindness following cerebral angiography: is this entity related to posterior reversible leukoencephalopathy? AJNR. American journal of neuroradiology, 25(2):252-6, feb 2004.

[164] Taylor I., Scheffer I., and Berkivic S. Occipital epilepsies: identification of specific and newly recognized syndromes. Brain, 126(4):753-769, apr 2003.

[165] Roebroeck A., Miller K. L., and Aggarwal M. Ex vivo diffusion MRI of the human brain: Technical challenges and recent advances. NMR in biomedicine, page e3941, jun 2018.

[166] Van Essen D. C., Smith S. M., Barch D. M., Behrens T. E. J., Yacoub E., Ugurbil K., and WU-Minn HCP Consortium. The WUMinn Human Connectome Project: an overview. NeuroImage, 80:62-79, oct 2013. 